\title{
Structural Investigations of Surfaces and Orientation-Specific Phenomena in Nanocrystals and Their Assemblies
}

by

Deborah Michiko Aruguete

B.S. (University of Chicago) 1998

A dissertation submitted in partial satisfaction of the

requirements for the degree of

Doctor of Philosophy

in

Chemistry

in the

GRADUATE DIVISION

of the

UNIVERSITY OF CALIFORNIA, BERKELEY

\author{
Committee in charge: \\ Professor A. Paul Alivisatos, Chair \\ Professor Gabor A. Somorjai \\ Professor Ronald Gronsky
}

Spring 2006 
The dissertation of Deborah Michiko Aruguete is approved:

\begin{tabular}{lc}
\hline Chair & Date \\
& \\
\hline & Date \\
& \\
\hline & Date
\end{tabular}

University of California, Berkeley

Spring 2006 
Structural Investigations of Surfaces and Orientation-Specific Phenomena in Nanocrystals and Their Assemblies

(C) 2006

by

Deborah Michiko Aruguete 


\title{
ABSTRACT \\ Structural Investigations of Surfaces and Orientation-Specific Phenomena in Nanocrystals and Their Assemblies
}

\author{
by \\ Deborah Michiko Aruguete \\ Doctor of Philosophy in Chemistry \\ University of California, Berkeley \\ Professor A. Paul Alivisatos, Chair
}

Studies of colloidal nanocrystals and their assemblies are presented. Two of these studies concern the atomic-level structural characterization of the surfaces, interfaces, and interiors present in II-VI semiconductor nanorods. The third study investigates the crystallographic arrangement of cobalt nanocrystals in self-assembled aggregates.

Crystallographically-aligned assemblies of colloidal CdSe nanorods are examined with linearly-polarized Se-EXAFS spectroscopy, which probes bonding along different directions in the nanorod. This orientation-specific probe is used, because it is expected that the presence of specific surfaces in a nanorod might cause bond relaxations specific to different crystallographic directions. Se-Se distances are found to be contracted along the long axis of the nanorod, while Cd-Se distances display no angular dependence, which is different from the bulk. Ab-initio density functional theory calculations upon CdSe nanowires indicate that relaxations on the rod surfaces cause these changes.

ZnS/CdS-CdSe core-shell nanorods are studied with Se, Zn, Cd, and S X-ray absorption spectroscopy (XAS). It is hypothesized that there are two major factors influencing the core and shell structures of the nanorods: the large surface area-to-volume 
ratio, and epitaxial strain. The presence of the surface may induce bond rearrangements or relaxations to minimize surface energy; epitaxial strain might cause the core and shell lattices to contract or expand to minimize strain energy. A marked contraction of $\mathrm{Zn}-\mathrm{S}$ bonds is observed in the core-shell nanorods, indicating that surface relaxations may dominate the structure of the nanorod (strain might otherwise drive the $\mathrm{Zn}-\mathrm{S}$ lattice to accommodate the larger $\mathrm{CdS}$ or CdSe lattices via bond expansion). EXAFS and X-ray diffraction (XRD) indicate that $\mathrm{Cd}-\mathrm{Se}$ bond relaxations might be anisotropic, an expected phenomenon for a rod-shaped nanocrystal.

Ordered self-assembled aggregates of cobalt nanocrystals are examined with transmission electron microscopy (TEM) and selected-area electron diffraction (SAED). SAED patterns from multilayered assemblies show that the nanocrystals have preferred crystallographic orientations. It is proposed that the nanocrystals are organized in a vortexlike or "loop-closing" arrangement, possibly due to magnetism. SAED and dark-field imaging used to investigate this hypothesis are presented, along with the data analysis. The effects of magnetism and nanocrystal faceting are discussed.

Professor A. Paul Alivisatos

Dissertation Committee Chair 
For my grandparents, Takako and Matsuso "Roy" Hashioka 


\section{TABLE OF CONTENTS}

LIST OF FIGURES....................................................vi

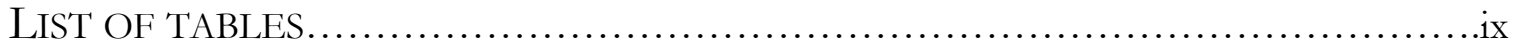

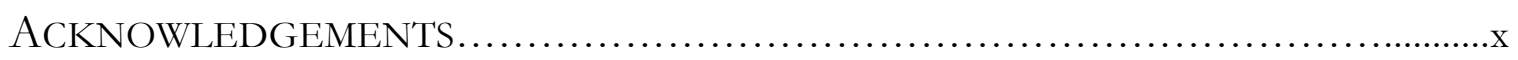

CHAPTER 1. INTRODUCTION TO COLLOIDAL NANOCRYSTALS AND THEIR

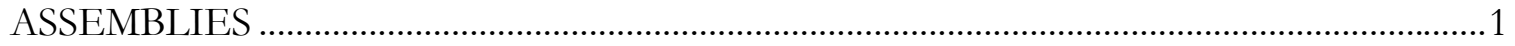

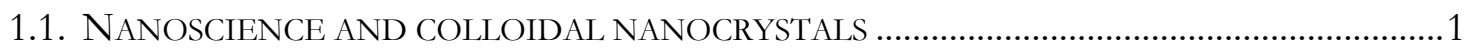

1.2. HIGHER-DIMENSIONAL COLLOIDAL NANOCRYSTALS AND THEIR SURFACES ................... 1

1.3. COLLOIDAL NANOCRYSTALS AND THEIR ASSEMBLIES............................................................

CHAPTER 2. INTRODUCTION TO X-RAY ABSORPTION SPECTROSCOPY (XAS)6

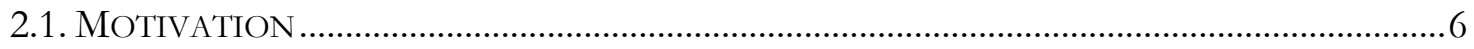

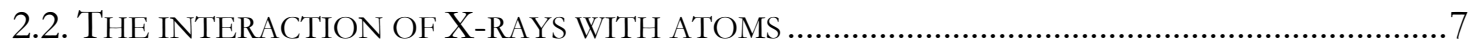

2.2.1. Absorption of an X-ray photon by a single atom ......................................................

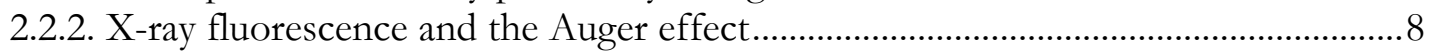

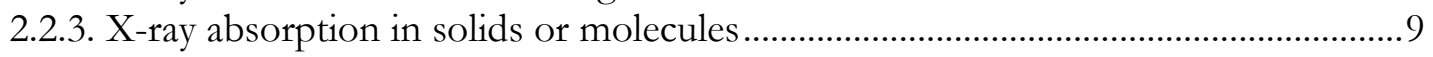

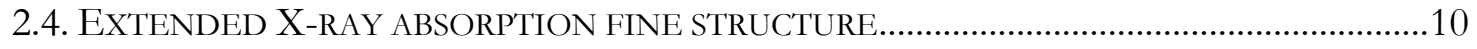

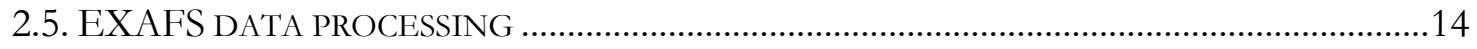

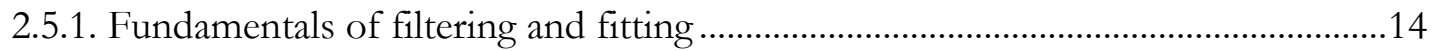

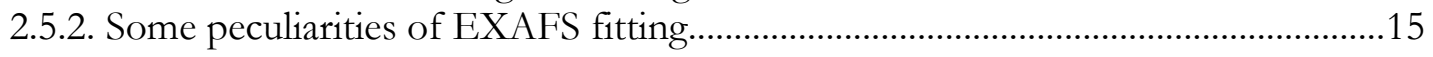

2.5.2.1. The perils of non-linear multivariable fitting ...................................................... 15

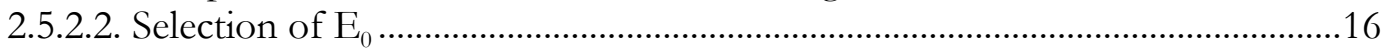

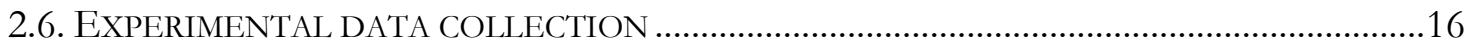

CHAPTER 3. POLARIZED EXTENDED X-RAY ABSORPTION FINE STRUCTURE (EXAFS) SPECTROSCOPY OF ORIENTED CDSE NANORODS ..................................18

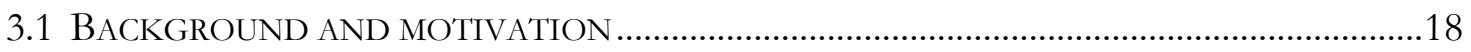

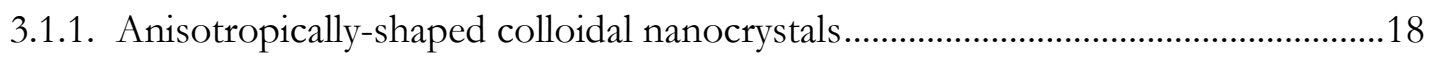

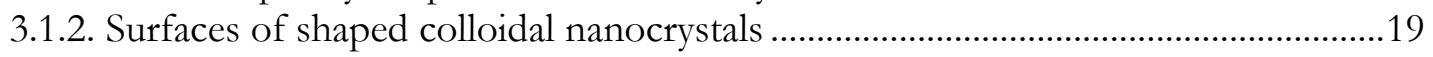

3.2. BULK AND NANOROD CADMIUM SELENIDE (CDSE): BASIC PROPERTIES .........................20

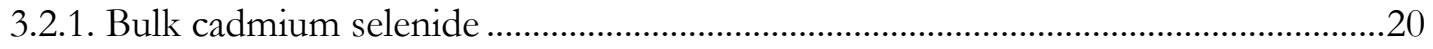

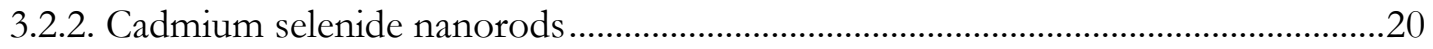

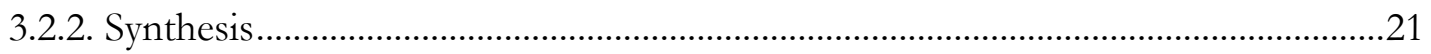

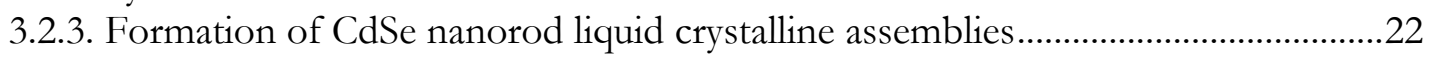

3.2. POLARIZED EXTENDED X-RAY ABSORPTION FINE STRUCTURE (EXAFS)

SPECTROSCOPY: BACKGROUND 


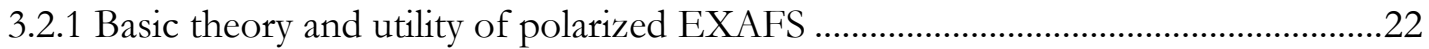

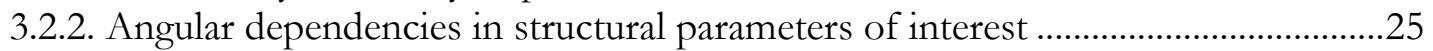

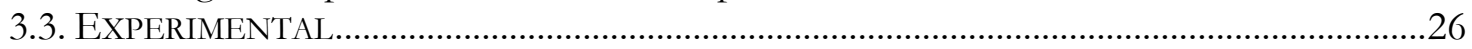

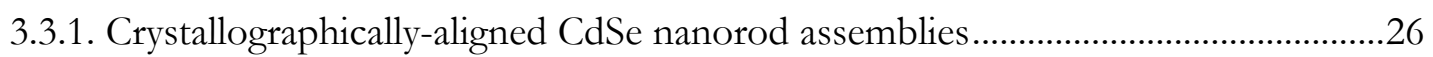

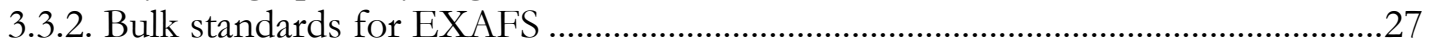

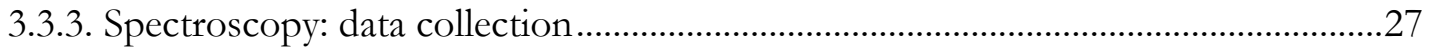

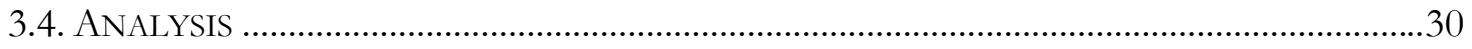

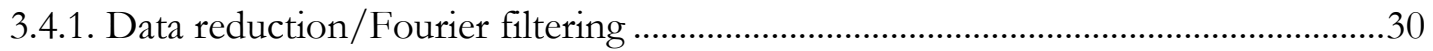

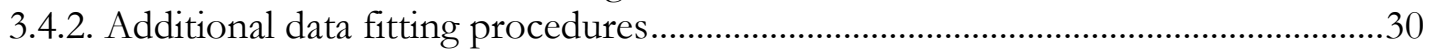

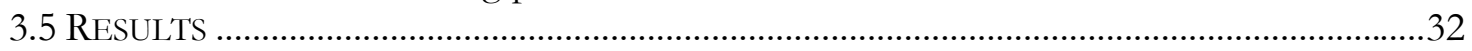

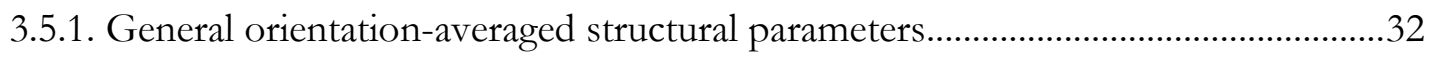

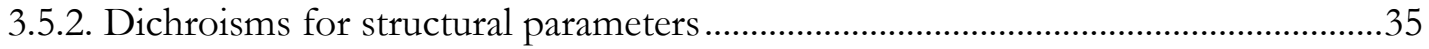

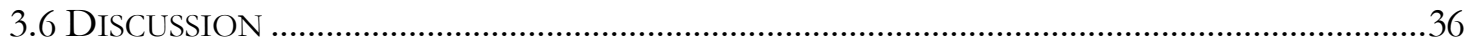

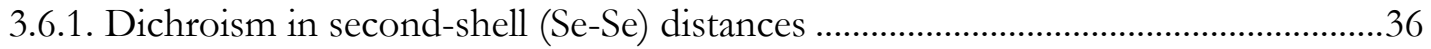

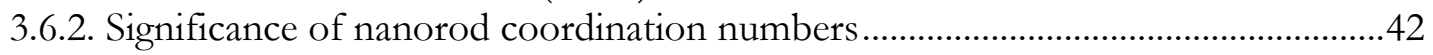

3.7. Potential CAVEAT: PRESENCE OF TWO FIRST-SHELL AND SECOND-SHELL

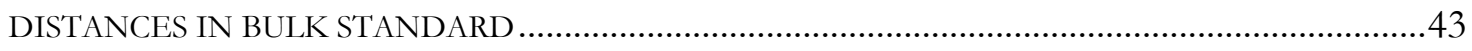

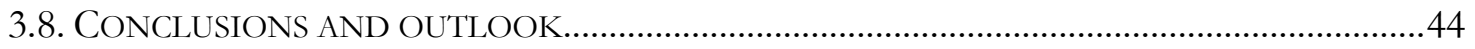

CHAPTER 4. X-RAY ABSORPTION SPECTROSCOPY OF CDSE/ZNS CORE-SHELL NANORODS

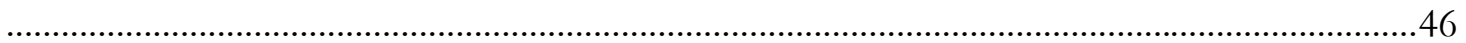

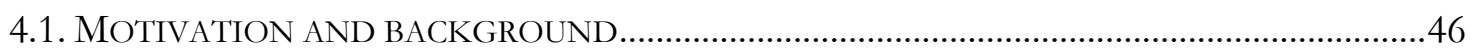

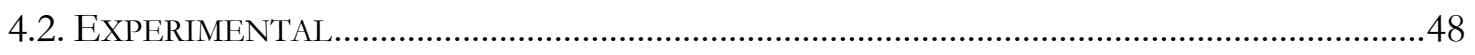

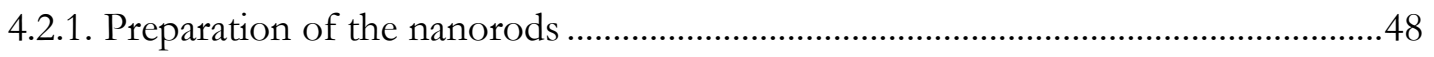

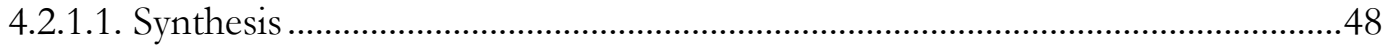

4.2.1.2. Photoannealing of core-shell nanorod samples..................................................51

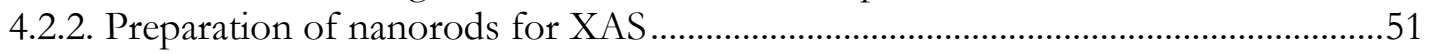

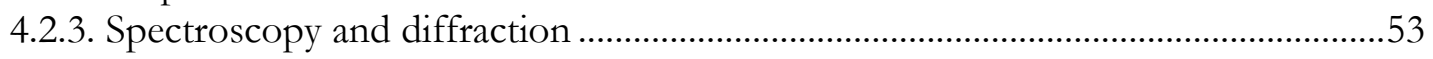

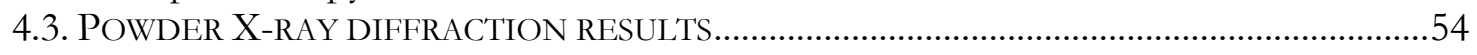

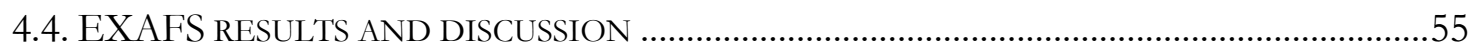

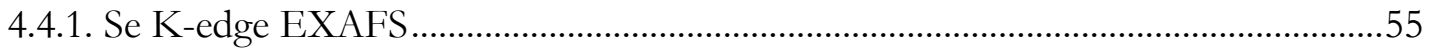

4.4.1.1. Se first shell distances (Cd-Se distances) ..........................................................56

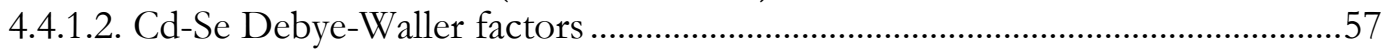

4.4.1.2.1. General contributions to disorder in materials ............................................5

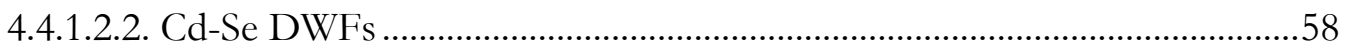

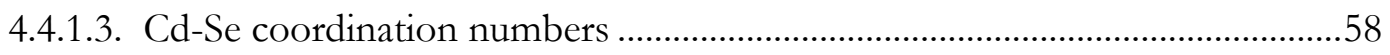

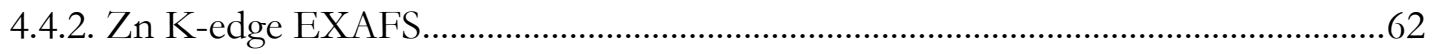

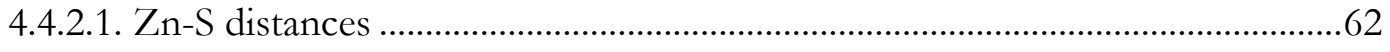

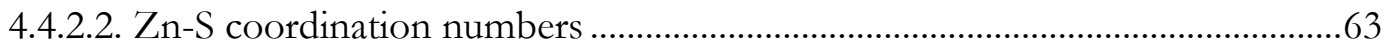

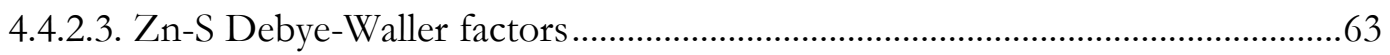

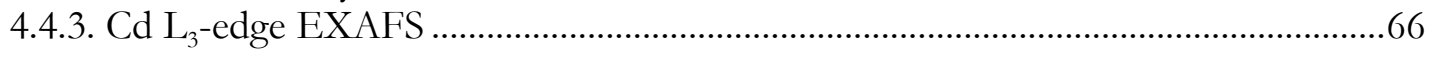

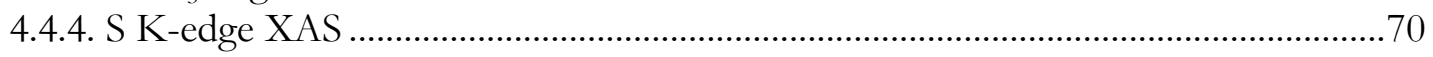

4.5. OUTLOOK AND CONCLUSIONS WITH RESPECT TO PREVIOUS WORK ON NANOSCALE

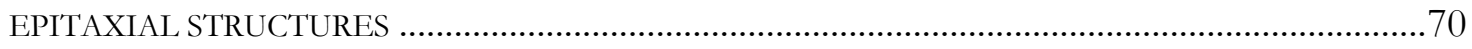


CHAPTER 5. INTRODUCTION TO ELECTRON DIFFRACTION ................................73

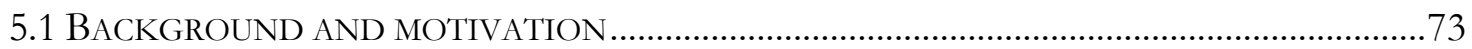

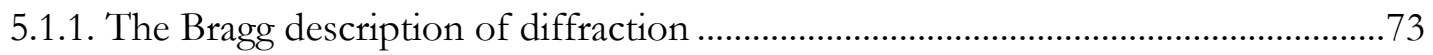

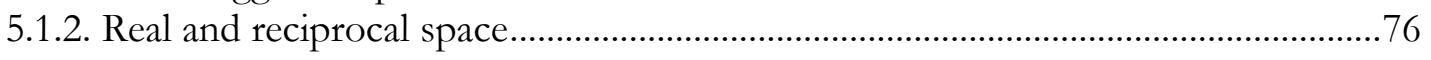

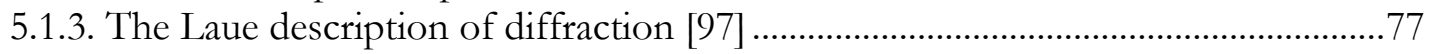

5.1.4. The Ewald sphere ...............................................................................................

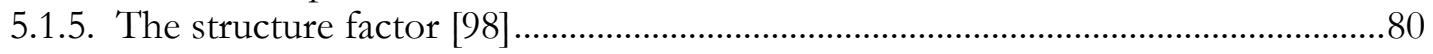

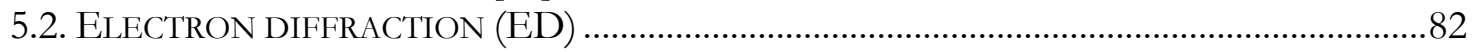

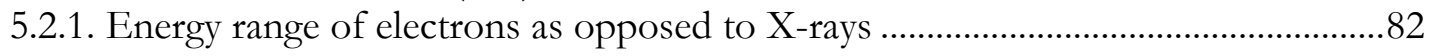

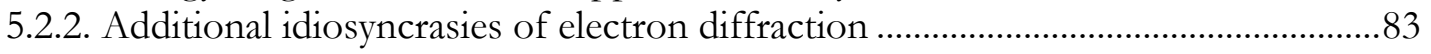

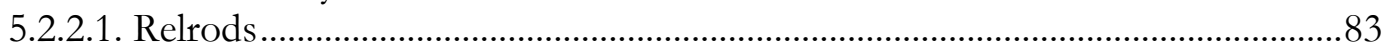

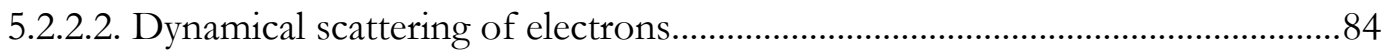

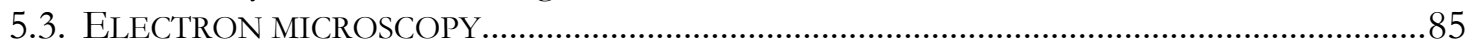

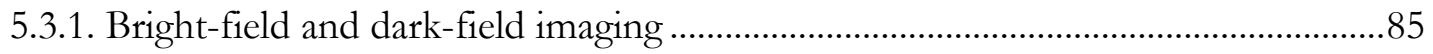

5.3.2. High resolution transmission electron microscopy (HRTEM)................................89

CHAPTER 6. CRYSTALLOGRAPHIC CHARACTERIZATION OF $\varepsilon$-COBALT

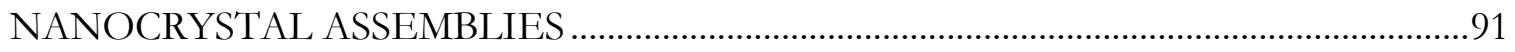

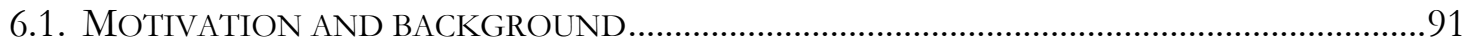

6.2. PREPARATION OF $\varepsilon$-COBALT NANOCRYSTALS AND THEIR ASSEMBLIES ...........................93

6.3. TRANSMISSION ELECTRON MICROSCOPY (TEM) AND SAED (SELECTED-AREA

ELECTRON DIFFRACTION) OF COBALT NANOCRYSTAL ASSEMBLIES ......................................95

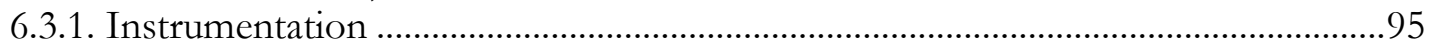

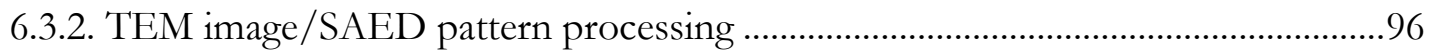

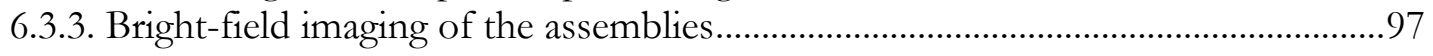

6.3.4. SAED from monolayer and multilayer assemblies ..................................................98

6.3.5. Dark-field imaging from multilayered assemblies.................................................... 104

6.3.6. High-resolution TEM (HRTEM) imaging of individual particles and their

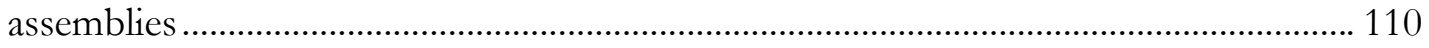

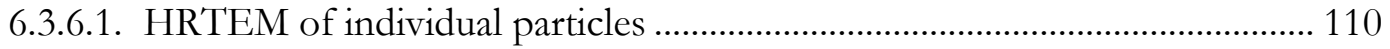

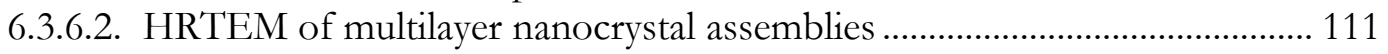

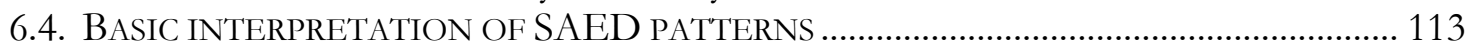

6.5. ANALYSIS OF TEXTURED PATTERNS FROM COBALT NANOCRYSTAL ASSEMBLIES ...... 116

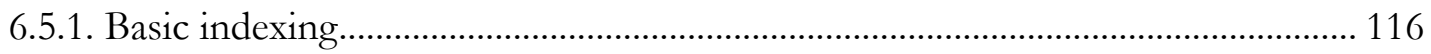

6.5.2. Hypotheses regarding crystallographic organization within the nanocrystal islands

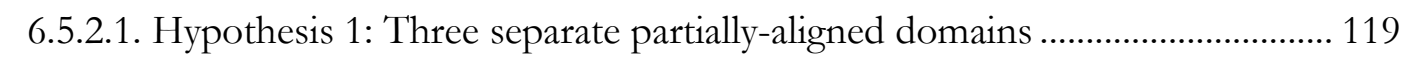

6.5.2.2. Hypothesis 2: Alignment along axis canted out of the sample plane........... 121

6.6. CAUSES OF CRYSTALLOGRAPHIC ORIENTATION IN COBALT NANOCRYSTAL

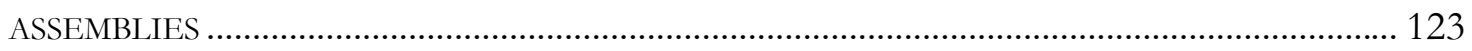

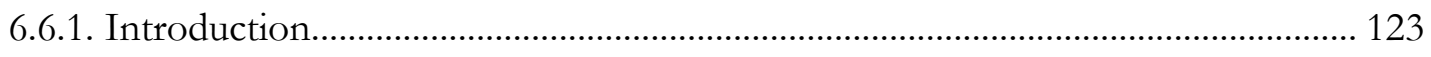

6.6.2. Magnetism and crystallographic alignment in nanocrystals................................. 123

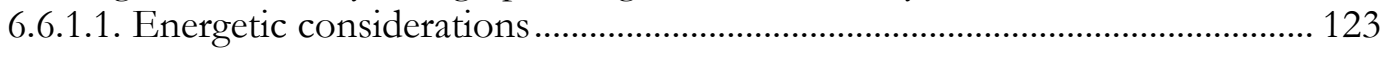




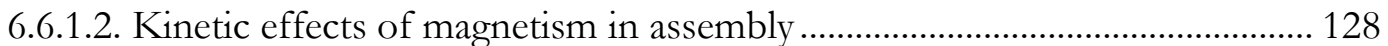

6.6.2. Van der Waals (or packing) forces: the role of faceting........................................ 130

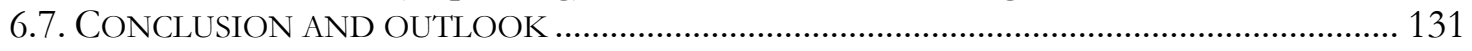

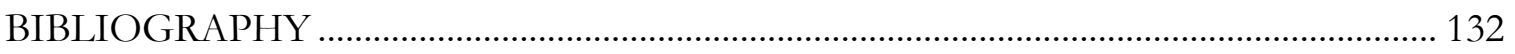

APPENDIX 1: IMAGE PROCESSING WITH ADOBE PHOTOSHOP 7.0 (PC) ......... 140

APPENDIX 2: INDEXING OF DIFFRACTION PAT'TERNS AND CROSS

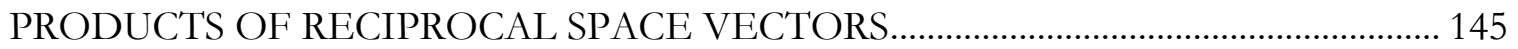

APPENDIX 3: INITIAL ATTEMPTS TO VERIFY A THREE-DOMAIN

HYPOTHESIS FOR THE NANOCRYSTAL ASSEMBLIES ……….................................. 155 


\section{LIST OF FIGURES}

2.1. Energy-level diagram of photoelectric effect for a single atom..........................................

2.2. Relaxation processes for photoexcited atoms. ..................................................................

2.3. Illustration of the different energy regimes in $\mathrm{X}$-ray absorption spectroscopy................11

2.4. Schematic of basic procedure for data processing...............................................................13

2.5. Schematic of EXAFS experimental setup ......................................................................17

3.1. Basic wurtzite structure, and a schematic image of a CdSe nanorod ..................................21

3.2. Diagram depicting preferential probing along different directions in a nanorod by

polarized EXAFS.

3.3. Schematic drawing of the experimental setup, from which both XRD and EXAFS data could be collected from a single domain of oriented nanorods .28

3.4. Sample holder for capillary.

3.5. Experimental pair distribution functions of bulk and nanorod CdSe, as well as likely surface oxides and selenium-tributylphosphine

3.6. Top views of unrelaxed and relaxed $2.4 \mathrm{~nm}$ diameter CdSe nanowire from DFT

calculations

3.7. Measured $1^{\text {st }}$ and $2^{\text {nd }}$-shell distances versus $<\cos ^{2} \theta>$, and calculated, $\cos ^{2} \theta$-weighted $1^{\text {st }}$ and $2^{\text {nd }}$-shell distances versus $\cos ^{2} \theta$

4.1. Schematic of the growth of a graded CdS/ZnS shell on a CdSe nanorod........................49

4.2. UV-visible absorption spectra from core and core-shell nanorods....................................50

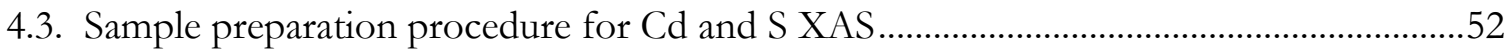

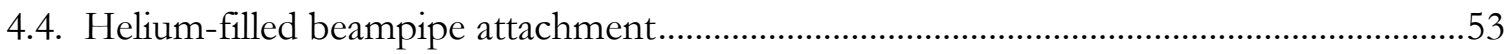


4.5. X-ray diffractograms from core and core-shell nanorods ................................................59

4.6. Fourier transform magnitudes from raw Se K-edge EXAFS data ....................................60

4.7. Structural parameters derived from Se K-edge EXAFS on core and core-shell nanorods .61

4.8. Fourier-transform magnitudes of Zn K-edge raw data.......................................................64

4.9. Physical parameters for first-shell $\mathrm{Zn}-\mathrm{S}$ bonds derived from $\mathrm{Zn}$ K-edge EXAFS..........65

4.10. Fourier-transform magnitudes of $\mathrm{Cd} \mathrm{L}_{3}$-edge raw data ...................................................68

4.11. First-shell physical parameters for Cd-S pairs derived from $\mathrm{Cd} \mathrm{L}_{3}$-edge EXAFS ..........69

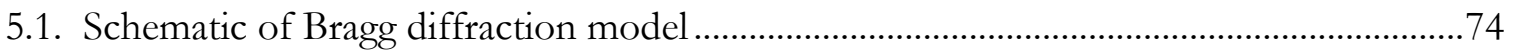

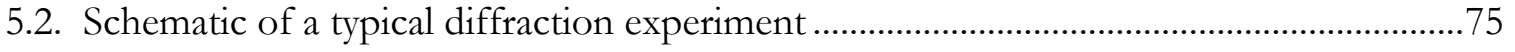

5.3. Schematic for discussion of Laue description of diffraction.............................................78

5.4. Schematic of a typical 2D cross-section of an Ewald sphere for X-rays...........................80

5.5. Schematic cross section of Ewald sphere for high-energy electrons ……..........................83

5.6. Materials with dimensions $<100 \mathrm{~nm}$ can result in rods (or other frequency

distributions) rather than discrete points in reciprocal space ....................................................84

5.7. Basic beam alignment for bright-field imaging versus dark-field imaging ........................87

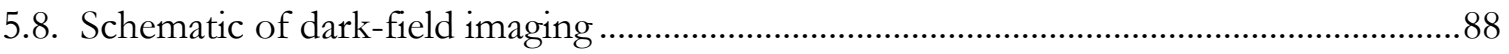

5.9. Typical HRTEM images of cobalt nanocrystals.................................................................89

6.1. Assemblies formed by drying cobalt nanocrystals ( $\varepsilon$-phase) in solution on a TEM grid93

6.2. Preparation of cobalt nanocrystal assemblies on TEM grids .............................................95

6.3. Examples of structures formed after deposition of cobalt nanocrystals ..........................97

6.4. TEM image of a monolayer of cobalt nanocrystals, and a typical SAED pattern from a monolayer 


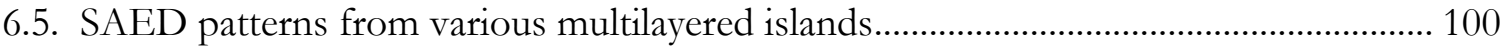

6.6. Typical SAED pattern from the center of a multilayer island, with indexing................. 101

6.7. Alternate SAED pattern from multilayer island, with indexing...................................... 101

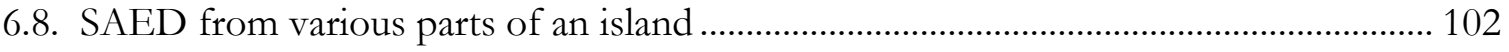

6.9. Small-angle diffraction from the lattice-based arrays of nanocrystals present in the

islands.

6.10. Alternate SAED pattern and corresponding dark field images .................................... 106

6.11. Schematic of possible crystallographic orientations of the nanocrystals within an island

6.12. Top row, left to right: Bright field image of an island approximately .......................... 108

6.13. Another example of dark-field data from a multilayered island .................................... 109

6.14. HRTEM images of three separate $\varepsilon$-cobalt nanocrystals ................................................. 110

6.15. Example of partial orientation on edge of island............................................................ 112

6.16. Another HRTEM image from an island, and the corresponding Fourier transform. 112

6.17. Two depictions of the zone axis for a crystal................................................................. 114

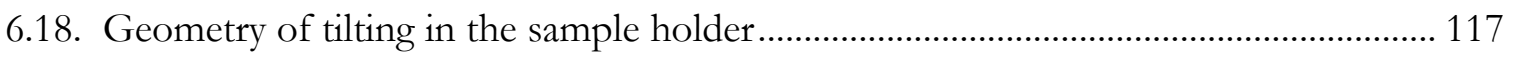

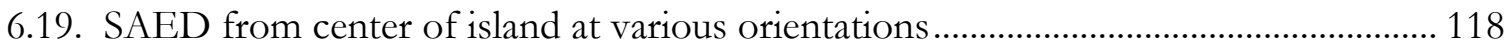

6.20. Images of different types of orientation in reciprocal space ....................................... 120

6.21. Hypothesized three-domain crystallographic arrangement of the cobalt nanocrystals in multilayered assemblies

6.22. Alternative hypothesized crystallographic arrangement, in which the nanocrystals all have a single axis (imperfectly fixed) which is oriented out of the sample plane

6.23. Results of energy calculations on lattices of classical dipoles. 


\section{LIST OF TABLES}

3.1 General structural parameters for bulk and nano CdSe (orientation-averaged) .........33

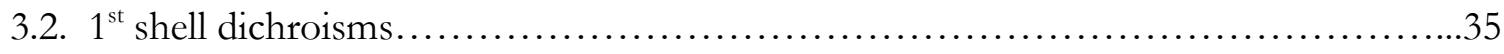

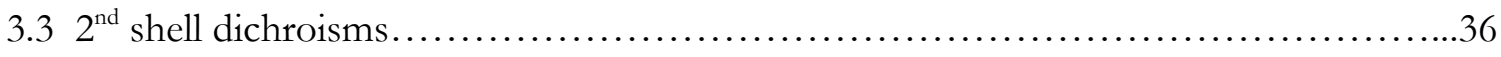

4.1 XRD peak position changes as a function of shell thickness......................54 


\section{ACKNOWLEDGEMENTS}

Graduate school has been a turbulent and bumpy journey for me, and I could not have survived it alone. It is impossible for me to express the depth of my gratitude to those who have helped me.

I would first like to thank my advisor, Paul Alivisatos, who "welcomed me in from the cold", as it were, when my first advisor lost tenure. By allowing me a great deal of freedom, he helped me develop into the independent scientist I am today. He had the patience to let me pursue projects which, while unexpectedly difficult, expanded my intellectual limits. Having confronted these challenges, I feel that I am prepared for what lies ahead.

Many of the best memories I will take with me from graduate school are from my fellow graduate students and colleagues at Berkeley, especially those from the Alivisatos group. Among these was Benjamin Boussert, one of the truest friends I've had out here in Berkeley. He helped me remain strong in the face of many trevails. His untimely death tore him out of our lives, but I will never forget him. Steve Shipman (nee Robertson), Christine Micheel, Stephen Prilliman all helped welcome me into the physical chemistry clique when I was making the transition from being a synthetic chemist in my early years at Berkeley. Delia Milliron, Emory Chan, Daniele Gerion, Alex Mastroianni, Josh Wittenberg, Stevo Hughes, Cody Folden, C.J. Lee, Keren Jacobs, Paul Trudeau, and Masahiro Ishigami are among the many people who have been great to know, both professionally and personally.

My collaborations with other students have been a great deal of fun. I am very happy to have worked with Liang-shi Li. In addition to having initiated the X-ray absorption 
collaboration that forms a great part of my dissertation, he was a wonderful mentor and friend. I have also worked closely with Can Erdönmez, who, with his patient teaching style, his brilliance, and his aberrant sense of humor enriched my life both scientifically and personally. Ben Boussert collaborated with me in my X-ray absorption studies of core-shell nanorods. Chapter 4, which details these studies, is dedicated to his memory.

Much of my work has involved collaborations with seasoned scientists outside of my research group, who have taught me a great deal of what I know. First, I must thank Dr. Matthew Marcus of the Advanced Light Source, Lawrence Berkeley National Laboratory (LBNL) for teaching me all about X-ray absorption spectroscopy, and for being a very hardworking collaborator. His tolerance of my never-ending stream of questions (and e-mails) was nothing short of amazing. Dr. Andrew Williamson (Lawrence Livermore National Laboratory) has also been a helpful collaborator, who took the time to explain his calculations to an experimentalist.

All work at the LBNL National Center for Electron Microscopy (NCEM), especially in its initial stages, was performed in collaboration with Dr. Tamara Radetic, an NCEM staff scientist. Dr. Radetic was responsible in large part for training me and my colleague, Dr. Can Erdönmez, in the use of the microscopes. I especially appreciated her rigorous discussions of our crystallographic data. Dr. Velimir Radmilovic, Dr. Ulrich Dahmen, and Dr. Mark Williamson of NCEM also provided useful discussions. Reena Zalpuri and Dr. Gordon Vrdoljak, scientific staff at the University of California Berkeley Electron Microscope Lab, trained us in the use of the FEI Tecnai 12.

My family has been a great font of strength for me during my time at Berkeley, offering unconditional love, as well as a non-academic perspective (and strange care 
packages). During my time at Berkeley, I have come to more fully appreciate how lucky I am to have such a fantastic family.

I am grateful to Keith Ball, my partner. Having gone through the doctoral process himself, he was extremely sympathetic and supportive, and incredibly patient. Other members of the Ball family, including Alpert Ball and Briar Ball, were also very helpful. I would like to acknowledge the help of two people in reviewing this dissertation: Can Erdönmez and Professor Ronald Gronsky (MSE). Both of them took the time to review it very carefully and helped to make it a better piece of work.

Last, but not least, I am thankful for all of the impediments I have faced, even if they often seemed to be unwarranted or insurmountable. Having overcome such difficulties makes the completion of my doctoral work an even sweeter accomplishment. 


\section{CHAPTER 1. INTRODUCTION TO COLLOIDAL NANOCRYSTALS AND THEIR ASSEMBLIES}

\subsection{Nanoscience and colloidal nanocrystals}

Nanoscience loosely refers to the study of materials with dimensions between 1 and $100 \mathrm{~nm}$. In this size regime many materials display novel size-dependent properties somewhere between that of bulk solids and molecules [1]. One type of nanometer-scale material under study is the colloidal nanocrystal. Colloidal nanocrystals are soluble nanometer-scale crystals which can be synthesized in a highly-controlled fashion, allowing for the precise tuning and systematic study of desired shape- or size-dependent properties [2]. In addition to being of interest for fundamental study, their solution processability and the ease of chemical surface modification makes nanocrystals attractive candidates for applications such as solar cells [3, 4], magnetic resonance imaging contrast agents [5], fluorescent biolabels [6], and gas sensors [7]. The studies described in this dissertation concern the physical properties of nanocrystals, in particular II-VI semiconductor nanocrystals and magnetic metallic nanocrystals.

\subsection{Higher-dimensional colloidal nanocrystals and their surfaces}

One of the major differences between nanocrystals and their bulk counterparts is that they often are largely composed of surface atoms. For example, in a $3 \mathrm{~nm}$ diameter spherical nanocrystal, close to half of the atoms are on the surface. The surface area-to-

volume ratio of a nanocrystal scales with size, approximately as $1 / r$, where $r$ describes a 
nanocrystal dimension, e.g. the radius of a spherical nanocrystal. Many properties of nanocrystals have been shown to depend upon the presence of the surface, or to vary with chemical modifications to their surfaces; these include optical properties [8], phase transitions [9], and catalytic reactivity [10].

A deeper understanding of nanocrystal physical properties necessitates good characterizations of their surface structures. For colloidal nanocrystals, surface characterizations have been limited mostly to studies of spherical nanocrystals [11-13]. Nevertheless, advances in synthetic chemistry have delivered many new higher-dimensional shapes of colloidal nanocrystals displaying interesting, potentially useful shape-dependent properties [14-16]. Very little is known about the basic chemical bonding or structure of the surfaces of these higher-dimensional nanocrystals [17].

Structural characterizations of both the surfaces and interiors of higher-dimensional nanocrystals, specifically colloidal II-VI semiconductor nanorods, are reported in chapters 3 and 4 of this dissertation. These studies were initial attempts to address how atomic bonding in nanocrystals might vary with shape. Extended X-ray absorption fine structure (EXAFS) spectroscopy was used to characterize the local environment around the atoms in the nanorods; the presence of the nanorods' surfactant coating makes the use of other surface probes difficult. A brief general introduction to X-ray absorption spectroscopy (XAS) and EXAFS is presented in chapter 2.

It is expected that the presence of specific surfaces in a nanorod would influence the geometry of the surface bonding, possibly causing bond relaxations specific to different crystallographic directions in the rod. In order to probe structure along different directions 
in the nanorods, polarized EXAFS was measured from samples of crystallographicallyaligned assemblies of CdSe nanorods; these experiments are detailed in chapter 3.

In chapter 4 , studies of the surface, interface, and interior structures of $\mathrm{ZnS} / \mathrm{CdS}$ CdSe core-shell nanorods are discussed. It is hypothesized that there are two major factors influencing the core and shell structures of the nanorods. First is the presence of the surface; as the surface area-to-volume ratios of the nanorods are large, bond relaxations minimizing surface energy might dominate the structure of the rods. Second is the epitaxial growth; the lattice mismatch between the CdSe core and the $\mathrm{ZnS} / \mathrm{CdS}$ shell could cause bond relaxations that would reduce epitaxial strain. These scenarios were explored with EXAFS measurements on the nanorods, which are presented. It is also expected that the shape might play a role in any relaxations, possibly causing phenomena specific to crystallographic directions in the rods. The possible effect of shape is discussed in light of both EXAFS and XRD measurements upon the core-shell nanorods.

Understanding the surface structures/compositions of these higher-dimensional nanocrystals offers two major benefits. First, just as with spherical nanocrystals, one can gain better insight regarding the origins of their physical properties. Second, surfaces are believed to be key to shape control in nanosynthesis, because they vary in their chemical reactivity and stability $[18,19]$; by inhibiting the growth of certain faces, different crystal morphologies can be produced. While currently many theories abound regarding nanocrystal growth, there is minimal direct physical evidence to substantiate any of them. Thus, careful characterization of higher-dimensional colloidal nanocrystal surfaces may improve the understanding and subsequent control of nanocrystal synthesis, as well as epitaxial growth in colloidal nanocrystal systems. 


\subsection{Colloidal nanocrystals and their assemblies}

Solely examining the individual properties of nanocrystals does not suffice when considering their future applications; their integration into practical devices must also be contemplated. One potential use for nanocrystals is as storage or signaling elements in computer circuitry, which may help to meet the demand for increasing miniaturization in computer devices [20-22]. Of particular interest for such systems is the so-called "bottomup" approach $[23,24]$, in which device components are directly constructed of molecules or nanometer-scale parts (as opposed to the "top-down" approach, in which lithography is used to etch a circuit pattern).

Given the possibility of such an approach, it is important to study how nanomaterials such as colloidal nanocrystals may be assembled into ordered architectures. Earlier studies have shown that nanocrystals with good size and shape distributions in solution can spontaneously assemble into ordered structures under drying [25-27]. This drying-mediated assembly is controlled according to particle-particle forces, particlesubstrate forces, and particle-solvent-forces [28-30]. As many are interested in the use of nanocrystals for data storage [22], there has been a particular focus upon the assembly of magnetic nanocrystals.

Chapter 6 features studies of assemblies formed by magnetic cobalt nanocrystals. Particle-particle forces may be particularly interesting for nanocrystals in this size regime (9 nm diameter); both magnetic forces and Van der Waals forces are believed to play an important role in influencing assembly. Transmission-electron microscopy (TEM) and selected area electron diffraction (SAED) were used to characterize the structures formed by 
the cobalt nanocrystals. Both TEM and SAED are reviewed in chapter 5 of this dissertation. SAED showed that for multilayered assemblies, the nanocrystals had preferred crystallographic orientations.

It is hypothesized that the cobalt nanocrystals are arranged in a vortex or loopclosing configuration. SAED patterns and dark field images are used to characterize the crystallographic arrangement of the nanocrystals in the assemblies. This data is presented, as well as the current state of data analysis, which is still ongoing. The potential effects of magnetism and nanocrystal faceting upon the nanocrystal assemblies are discussed. 


\section{CHAPTER 2. INTRODUCTION TO X-RAY ABSORPTION SPECTROSCOPY (XAS)}

\subsection{Motivation}

The structural characterization of materials is a fundamental component of materials science. Structure is key to the identification of many materials. It is also critical for understanding the origins of a material's physical behavior.

Colloidal nanocrystals, due to their often poorly understood novel physical properties, necessitate detailed structural investigations. Generally, colloidal nanocrystals have a very high surface area-to-volume ratio. Thus it behooves us to not only study their interior structures, but their surfaces. Indeed, for colloidal nanocrystals, there are multiple examples in which chemical surface modifications have influenced their electronic behavior.

Unfortunately, a detailed characterization of nanocrystal surfaces, especially with respect to their atomic geometry, is far from simple. The standard "workhorse" methods of X-ray/electron diffraction and high resolution electron microscopy primarily offer information about the periodic part of the nanocrystals, namely, their interiors. Surface science techniques such as low energy electron diffraction or scanning tunneling microscopy are foiled by the presence of capping surfactants, as well as the fact that nanocrystals are not atomically flat (for a large enough area).

Luckily, there is a structural probe that has allowed us to examine colloidal nanocrystal surfaces: X-ray absorption spectroscopy (XAS). XAS can determine structural features such as the extent of atomic coordination on surfaces, bond distances, and 
structural disorder [31]. This chapter has a basic background of X-ray absorption spectroscopy, as well as a review of the extensive data analysis involved.

\subsection{The interaction of $\mathrm{X}$-rays with atoms}

\subsubsection{Absorption of an $\mathrm{X}$-ray photon by a single atom}

Before discussing actual X-ray absorption (and fluorescence) spectroscopy, a basic understanding of X-ray-atom interactions is necessary. Consider a single isolated atom absorbing an X-ray, which is simply light of an energy ranging from 1-500 keV . What happens is the well-known photoelectric effect [32], depicted in figure 2.1. The energy from the X-ray is transferred to a core-level electron. Thus, the energetically excited state, or final state of this system consists of an atom with a core hole, or an empty electronic state, and an electron that has been excited out of the atom into the continuum [33].

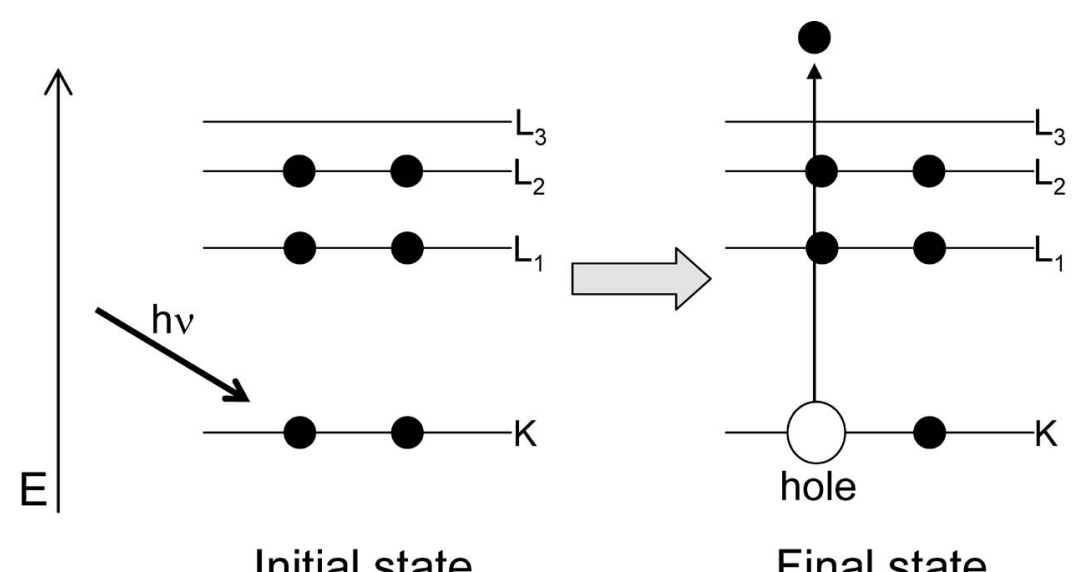

Figure 2.1. Energy-level diagram of photoelectric effect for a single atom, showing the initial state of the atom before excitation and the final state of the atom. 
Traditionally when using X-ray techniques, the core levels are not referred to as $1 \mathrm{~s}$, $2 \mathrm{p}, 3 \mathrm{~d}$, etc. but rather as $\mathrm{K}, \mathrm{L}$, and $\mathrm{M}$. K corresponds to the $1 \mathrm{~s}$ level, $\mathrm{L}$ corresponds to the $2 \mathrm{~s}$ and $2 \mathrm{p}$ levels, and $\mathrm{M}$ the $3 \mathrm{~s}, 3 \mathrm{p}$, and $3 \mathrm{~d}$ levels.

\subsubsection{X-ray fluorescence and the Auger effect}

A consideration of the matter-X-ray interaction does not end with the absorption event. The energetically excited atom cannot remain in that state indefinitely and has to energetically relax. There are two means by which this can occur [33], illustrated in figure 2.2. First, an electron from a higher energy state can fill the core hole, and release an X-ray photon with the excess energy. This process is known as X-ray fluorescence. Second, the atom can relax via a non-radiative process known as the Auger process. In this process, a higher-energy electron fills the core hole, and the remaining energy from this electron excites another electron to the continuum.
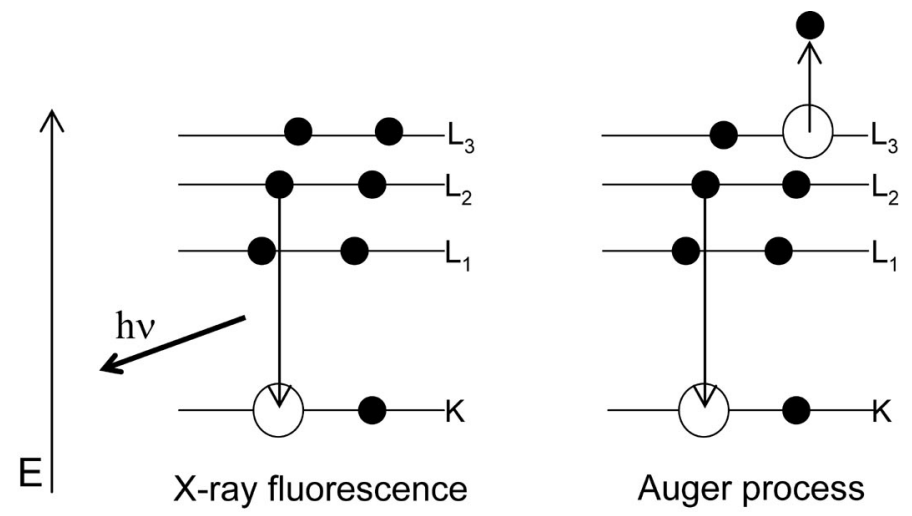

Figure 2.2. Energy-level diagram depicting relaxation processes for photoexcited atoms. 


\subsubsection{X-ray absorption in solids or molecules}

X-ray absorption by a compound can be described according to the Beer-Lambert Law $[34,35]$, which is as follows:

$$
I=I_{0} e^{-\mu(E) t}
$$

where $I_{0}$ is the incident X-ray intensity upon a sample, $I$ is the transmitted X-ray intensity, $t$ is the thickness of the sample, and $\mu$ is the linear absorption coefficient. X-ray absorption is simply the modulation in $\mu$ with respect to X-ray energy.

Modulations in $\mu$ occur when an atom in a compound, and not in a vacuum, interacts with an X-ray. The absorption coefficient $\mu$ is proportional to the probability of an electron being excited from a core-level state to a continuum state. This probability can be expressed according to Fermi's Golden Rule [36, 37], in which

$$
\mu \propto P=\frac{2 \pi}{\hbar}\left|\left\langle\psi_{i}|H| \psi_{f}\right\rangle\right|^{2} \rho\left(E_{f}\right),
$$

where $\psi_{i}$ and $\psi_{f}$ are the initial and final states of the photoelectron, respectively, $H$ is the Hamiltonian describing the interaction of the X-ray with the atom, and $\rho\left(E_{f}\right)$ is the density of states at the final energy of the photoelectron.

Let us consider what the final state of the emitted photoelectron will be when the emitting atom is surrounded by neighboring atoms. Given particle-wave duality, we can envision (as well as mathematically express) the photoelectron as an outgoing spherical wave. This spherical wave encounters the potentials of the surrounding atoms. A scattering process is established, leading to a general pattern of constructive and destructive interference. The final state of this photoelectron wave depends upon the geometric 
arrangement and composition (atoms of different elements have different sizes and masses) of its surroundings. Per equation 2.2., a change in $\psi_{f}$ will change $\mu(E)$. Herein lies the use of X-ray absorption as a structural probe.

\subsection{Extended X-ray absorption fine structure}

$\mathrm{X}$-ray absorption is measured by measuring the $\mathrm{X}$-rays transmitted through the sample. What we ultimately measure is $\mu(E)$; a typical example of a selenium XAS spectrum is shown in figure 2.3. This spectrum has several features to be noted. First, we note the sharp jump in absorbance, $\Delta \mu_{0}$, also known as an "edge", which corresponds to the core shell electron binding energy (the minimum energy necessary to excite an electron to the continuum). This spectrum is broken down into two regimes [31]. X-ray absorption near edge spectroscopy (XANES) concerns the spectrum $\sim 50-100 \mathrm{eV}$ above the edge jump (hence, "near edge"). Extended X-ray absorption fine structure (EXAFS) spectroscopy concerns the energy range above that of XANES. 


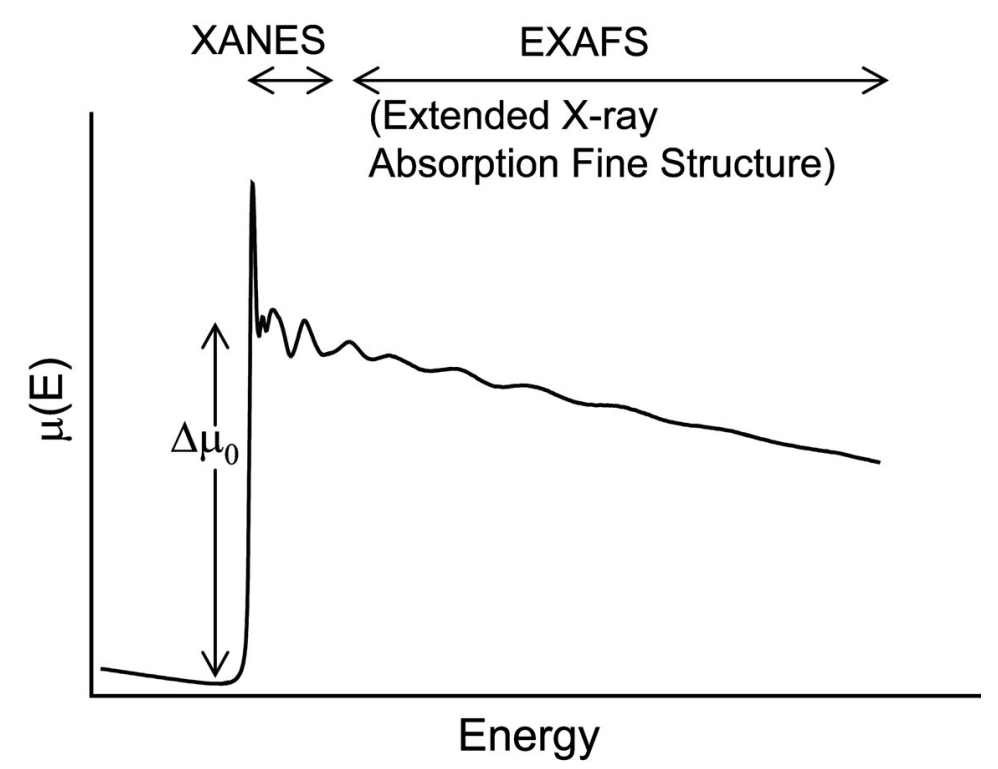

Figure 2.3. Illustration of the different energy regimes in X-ray absorption spectroscopy.

The EXAFS energy regime was primarily used for the XAS studies described in this dissertation. Photoelectrons in the EXAFS energy regime are mostly subjected to single scattering events. The information that can be derived from EXAFS is thus primarily geometrical and easily extracted from spectra [31]. EXAFS has been extensively used to obtain detailed information regarding local atomic structure. The EXAFS signal is specifically the oscillatory part of the spectrum (remember, for an isolated atom there are no surrounding atoms, which would result in a featureless spectrum), and is expressed as

$$
\chi(E)=\frac{\mu(E)-\mu_{0}(E)}{\Delta \mu_{0}}
$$

in which $\mu_{0}(E)$ is the featureless atomic absorption background, approximated by a spline function, and $\Delta \mu_{0}$ is the edge jump (see Fig 2.3). As we are dealing with an interference- 
based phenomenon, it is convenient to express the EXAFS signal in terms of the photoelectron momentum $k$, which is

$$
k=\sqrt{\frac{2 m\left(E-E_{0}\right)}{\hbar}},
$$

where $E_{0}$ is the energy at which the edge jump occurs and $m$ is the mass of the electron.

The full EXAFS equation, which is a summation of contributions from all atoms surrounding the central emitting atom is

$$
\chi(k)=\sum_{j} \frac{N_{j} f_{j}(k) e^{-2 k^{2} \sigma_{j}^{2}} e^{\frac{-2 R_{j}}{\lambda(k)}}}{k R_{j}^{2}} \sin \left[2 k R_{j}+\delta_{j}(k)\right]
$$

where $R_{j}$ is the distance to a neighboring atom $j, N_{j}$ is the coordination number of the neighboring atom $j, \sigma_{j}^{2}$ is the mean-square disorder of neighbor distance, and $\lambda$ is the mean free path of the photoelectron. $f_{j}(k)$ and $\delta_{j}(k)$ are photoelectron scattering properties of a given atom $j$, generally referred to as the amplitude and phase terms, respectively. The terms in this equation that limit EXAFS to being a local probe are the $1 / R_{j}^{2}$ term and the wavedampening term $e^{-\frac{2 R_{j}}{\lambda(k)}}(\lambda(k)<25 \AA$ for the EXAFS range of energies).

The expression for the EXAFS signal illustrates one of the primary caveats of the EXAFS technique - namely, that the signal is an averaged signal. Thus, while EXAFS is arguably the best tool for examining bonding distances and atomic coordination on colloidal nanocrystal surfaces (as described in section 2.1 of this chapter), it is not perfect, because the surface signal is convolved with that from atoms in the nanocrystal interior. Even electron yield detectors cannot circumvent this problem, because the nanocrystals being probed are on the length scale of the electron escape depths. The only way to confront this problem of 
the signal convolution is to compare the EXAFS results with those from a model. The use of models is discussed further in chapter 3.
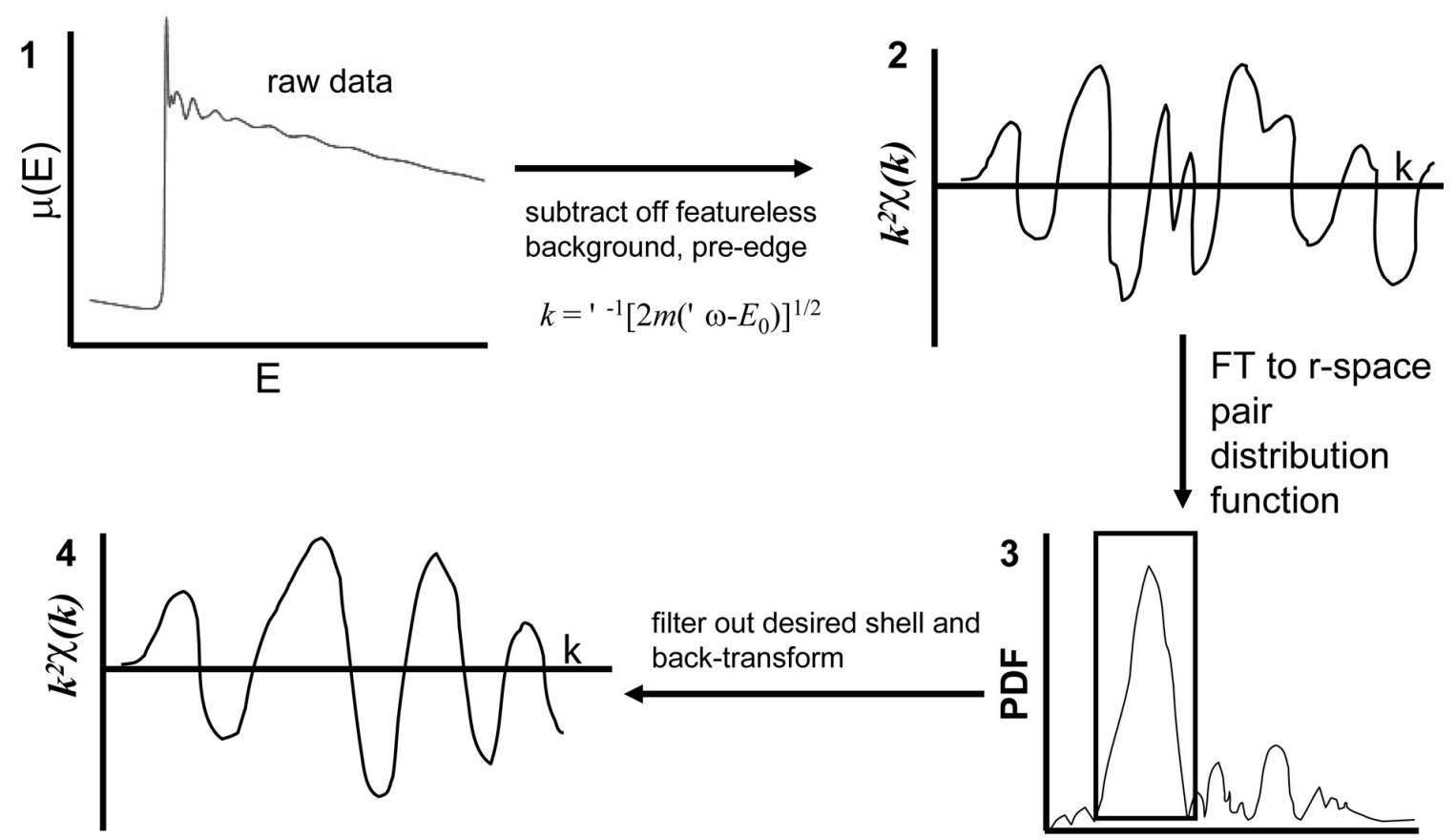

Fit filtered data to derive parameters

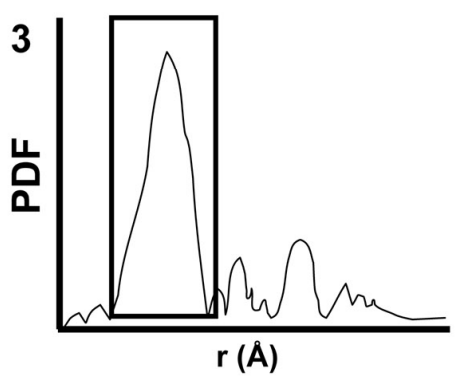

Figure 2.4. Schematic of basic procedure for data processing. 


\subsection{EXAFS data processing}

\subsubsection{Fundamentals of filtering and fitting}

Obtaining the function $\chi(k)$ requires processing the raw data to a substantial degree, as illustrated in figure 2.4. First, the featureless atomic absorption background is subtracted from the raw energy-space data (shown in figure 2.4, part 1) to isolate the oscillatory component, and the data is converted to $k$-space (fig. 2.4, part 2). This raw $\chi(k)$ function is multiplied by $k^{n}$ (for the analyses in this dissertation, $n=2$ was used) to help emphasize the high- $k$ parts of the signal.

As is clear from equation 2.4, this raw EXAFS signal contains contributions from first, second, and even third nearest neighbors (a.k.a. shells). To avoid dealing with all neighboring atoms simultaneously, $\chi(k)$ is filtered to isolate components of the oscillation corresponding to a particular set of neighbors. The EXAFS data is Fourier transformed from $k$-space to a real space function with both imaginary and real components. The real component (amplitude) for a sample of CdSe is displayed in part 3 of figure 2.4, with peaks corresponding to significant frequency components of the signal, namely, interatomic distances. By multiplying the EXAFS signal by a window function, these components can be selected out of the EXAFS signal. For example, in part 3 of figure 2.4, the first shell (first nearest-neighbor scattering) signal is being isolated. This real-space filtered function is then back-transformed to a filtered k-space function (figure 2.4, part 4). Thus, when equation 2.4 is fit to this filtered data, we need only to account for the first shell contributions to the 
signal. Another part of the data processing can also include truncating $\chi(k)$ at high $k$-values to remove completely obscured by noise.

In order to extract the physical parameters of interest (interatomic distances, coordination environments, degree of disorder) from $\chi(k)$ data, the specific photoelectron scattering functions $f_{j}\left(k_{e}\right)$ (amplitude) and $\delta_{j}(k)$ (phase) corresponding to the surrounding scattering atoms are required. They can be obtained either via calculations, most commonly performed using the software package FEFF [38], or by measuring the EXAFS on known standards. For most of the work in this dissertation, scattering functions were derived from empirical data on well-characterized standards.

All data processing and fitting were performed with Labview-based software written by Dr. Matthew Marcus of beamline 10.3.2. (See http://xraysweb.lbl.gov/uxas/Index.htm for further information about the software.)

\subsubsection{Some peculiarities of EXAFS fitting}

\subsubsection{The perils of non-linear multivariable fitting}

One of the primary problems with non-linear multivariable data fitting is that there is not one unique solution, or minimum in error space. When fitting, it is possible to fall into a false solution, rather than finding the solution that universally has the lowest possible fitting error. There are two major ways to avoid this, which were used for the data in this dissertation. First, one can start by fitting using reference data $\left(f_{j}(k)\right.$ and $\left.\delta_{j}(k)\right)$ that are very similar to the sample. Second, one can try to fit by inputting different starting values for parameters and checking to see if the program still converges onto the same solution. In 
essence, one should only use EXAFS when one already has a fairly good idea of what sorts of structures will be present.

\subsubsection{Selection of $E_{0}$}

$E_{0}$ is the energy at which the edge jump occurs. The determination of its value has been a matter of discussion [34], as the actual value is ill-defined due to the finite lifetime of the photoelectron and core hole. In other words, when one selects $E_{0}$, one is choosing the location of the Fermi level for a system, which may not be well-defined.

The values of $E_{0}$ selected for various analyses were based upon those selected for the known standards against which the data was fit. The Manceau method was utilized for selecting $E_{0}[39]$. Care was taken to be consistent in the selection of a value for $E_{0}$.

\subsection{Experimental data collection}

As EXAFS requires a source of X-rays with a continuous and tunable spectrum, generally all of it is measured at synchrotron sources. Synchrotron sources also have the high intensity and energy resolution necessary for such experiments. All XAS discussed in this dissertation was conducted at the Advanced Light Source, Lawrence Berkeley National Laboratory, Berkeley, CA, at beamline 10.3.2 in close collaboration with Dr. Matthew Marcus, chief beamline scientist [40]. Absorption spectra were measured using ion chamber detectors for both the transmitted intensity $I$ and the incident intensity $I_{0}$. Fluorescence spectra were measured using a solid-state germanium detector which detects energy for a specified emission energy range, depending on the edge being probed. The basic beamline 
set-up is illustrated in figure 2.5. Further details will be addressed in the following chapters detailing the experiments performed.

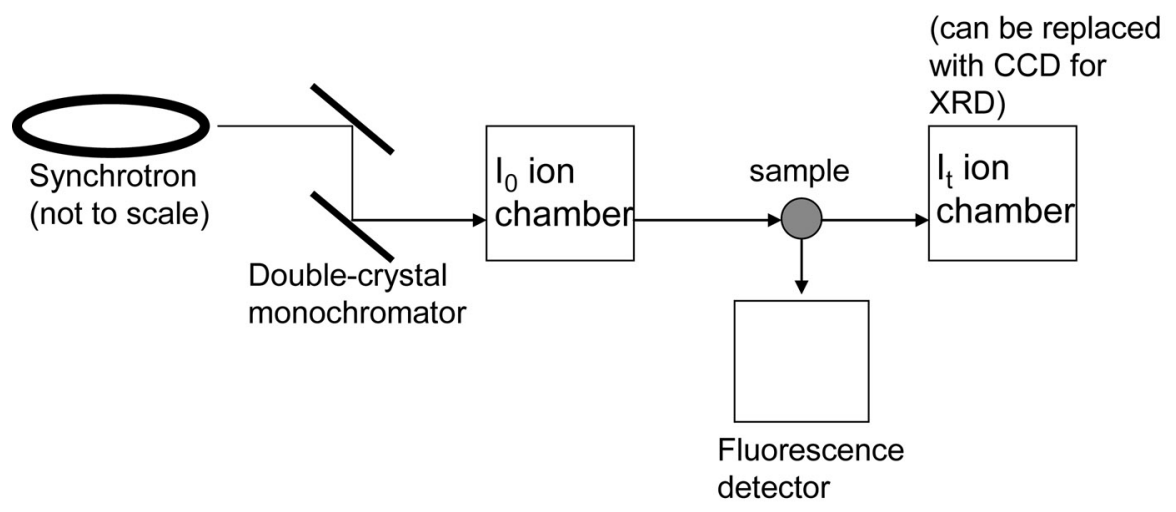

Figure 2.5. Schematic of EXAFS experimental setup. 


\section{Chapter 3. Polarized Extended X-RAy Absorption Fine STRUCTURE (EXAFS) SPECTROSCOPY OF ORIENTED CDSE NANORODS}

\subsection{Background and motivation}

\subsubsection{Anisotropically-shaped colloidal nanocrystals}

Many studies of colloidal nanocrystals have focused upon spherical nanocrystals, often (especially for semiconductors) referred to as quantum dots. Recently, synthetic chemistry has advanced far beyond the simple sphere; colloidal nanocrystals are available as rods [18, 41], disks [42], triangles [43], tetrapods [15], hollow spheres [44], and stars [45], to name a few shapes. Shaped nanocrystals often have novel physical properties different from those of their spherical counterparts which are of interest for both fundamental study and practical applications. For example, Au and CdSe nanorods, which respectively scatter and emit linearly polarized light, can be used as biolabels that provide information regarding the orientation of biomolecules [46][47]. CdSe nanorods have been used in a light-emitting diode that produces linearly polarized light [48]. CdTe tetrapods have been tested for use in solar power cells [3]; their geometry is considered advantageous for the better conduction of electrons and holes in the cells. Shape can also influence the behavior of magnetic nanomaterials, which may be used for data storage and signaling applications; indeed, different shapes can help to enhance or alter the magnetic anisotropy [49, 50]. 


\subsubsection{Surfaces of shaped colloidal nanocrystals}

Despite the surge in synthetic and physical research on shaped nanocrystals, their surfaces have not been well-examined. Nevertheless, surfaces are key to answering two major questions about shaped nanocrystals. First, what is the origin of their physical properties? As a large fraction of atoms for a shaped nanocrystal can be on the surface, a better understanding of the surface structure can benefit our physical knowledge. Second, why do shaped nanocrystals grow they way they do? It is generally agreed that surfaces play a large role in the control of nanocrystal morphology. Different crystal faces are believed to vary with regards to chemical reactivity and energetic stability; therefore, certain faces grow more quickly, resulting in anisotropic shapes[18,51,52]. The literature on nanocrystal synthesis is vast, but there exist few physical studies to confirm various theories regarding growth mechanisms. While organic chemistry has a framework for synthetic mechanisms (namely the so-called "electron-pushing" mechanisms), much of nanochemistry is still highly empirical.

This chapter describes efforts to learn about the surface structure and composition of a model anisotropic nanocrystal system-CdSe nanorods. It was expected that in nanorods, due to the presence of certain crystal faces, there might be bonding changes specific to crystallographic direction. Therefore, polarized extended X-ray absorption fine structure (EXAFS) spectroscopy was used to probe structure along different directions in the nanorods. 


\subsection{Bulk and nanorod cadmium selenide (CdSe): basic properties}

\subsubsection{Bulk cadmium selenide}

Bulk cadmium selenide is a semiconductor with a $1.74 \mathrm{eV}$ bandgap (direct) [53]. It can assume the wurtzite crystal structure, shown in figure 3.1. Its bonding is almost perfectly tetrahedral; the bonds along the $c$-axis are elongated by $0.005 \AA$ compared to the bonds along the $a$-/ $b$-axes [54]. The average bond distance (Cd-Se) in cadmium selenide is $2.63 \AA$; the Se-Se distance is $4.3 \AA[54]$.

\subsubsection{Cadmium selenide nanorods}

The cadmium selenide nanorods have been confirmed via XRD to have the hexagonally-symmetric wurtzite crystal structure [18]. They are grown such that the long axis is coincident with the [001] crystallographic direction, or the c-axis (figure 3.1). Highresolution transmission electron microscopy (HRTEM) has shown that the nanorods have only a few stacking faults per rod and are highly crystalline [18]. The optical absorption and emission of the CdSe nanorods have been shown to be highly dependent upon their width and length $[14,55]$. As with quantum dots, due to the spatial confinement of electrons and holes, their bandgaps are greater than $1.74 \mathrm{eV}$. Furthermore, the emission from the nanorods is polarized along the long axis [14]. 

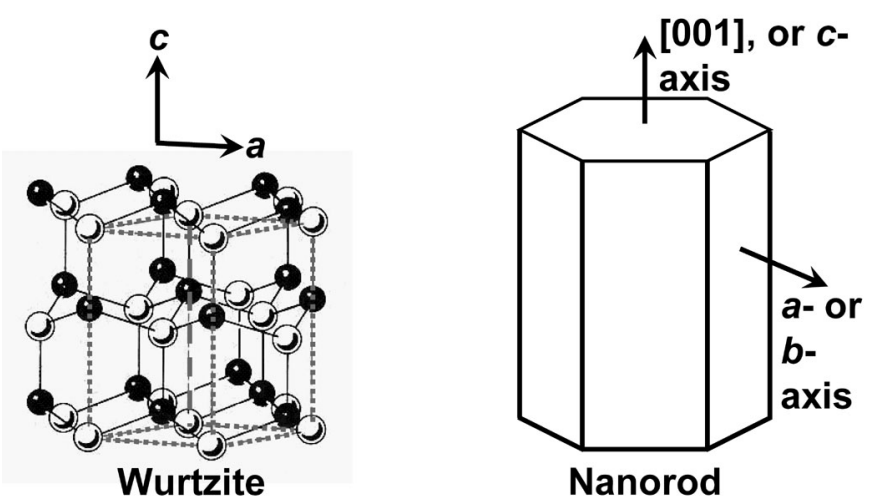

Figure 3.1. Left: Basic wurtzite structure, with unit cell outlined with gray dotted lines. Right: Schematic image of a CdSe nanorod. Note the alignment of the various crystallographic axes with the rod axes. Wurtzite image from http://www.metafysica.nl/ontology/wurtzite_1.jpg.

\subsection{Synthesis and assembly of CdSe nanorods}

\subsubsection{Nanorod synthesis}

The particular CdSe nanorod synthesis used for this study has been described in a previous paper [41] and Alivisatos group dissertation [56], and will only be briefly discussed here. The nanorods used for these studies were synthesized by Dr. Liang-shi Li, a former graduate student in the Alivisatos group. The basic synthesis involves the thermal decomposition of organometallic precursors (dimethyl cadmium, seleniumtributylphosphine) in hot, liquefied surfactants (trioctylphosphine oxide, alkylphosphonic acids) under air-free and water-free conditions. The precursors, upon decomposition, saturate the solution with molecular monomers, which leads to the nucleation and growth of nanocrystals. The surfactants in the reaction control the growth rates and morphology, as 
well as serving as capping or passivating agents for the finished nanocrystals. The size of the nanocrystals was measured using both TEM and UV-visible spectroscopy.

\subsubsection{Formation of CdSe nanorod liquid crystalline assemblies}

A fascinating property of CdSe nanorods is their ability to form crystallographicallyaligned assemblies [41, 57]; indeed, it is this property that has enabled the structural characterizations detailed in this chapter. At high concentrations, solutions of monodisperse, high aspect ratio nanorods spontaneously form liquid crystalline assemblies in which the rods are aligned such that their $c$-axes (long axes) are parallel to each other. The liquid crystalline phases are nematic, meaning that while the $c$-axes of the rods are aligned parallel to each other, the rods do not form rows (their centers of gravity are not aligned with each other). The alignment of the nanorods has been confirmed via optical microscopy, X-ray diffraction, and small angle $\mathrm{X}$-ray scattering.

\subsection{Polarized extended X-ray absorption fine structure (EXAFS) spectroscopy: background}

\subsubsection{Basic theory and utility of polarized EXAFS}

While the utility of EXAFS for nanocrystal surfaces was established earlier, the specifics of measuring EXAFS from anisotropic nanocrystals, especially nanorods, were not discussed. With an anisotropic system such as a nanorod, there are many questions concerning the effect of the overall shape upon the atomic-level structure. For example, how does confinement in a single dimension influence nanorod surface reconstructions? 
Are they different along the confined direction (short axis of the rods) as opposed to the unconfined direction (long axis of the rods)? What do the ends of the rods look like versus the sides? From the surface composition or coordination, can we infer anything regarding the growth mechanism?

Luckily EXAFS, when applied to a crystallographically-oriented sample, can offer orientation-specific information. This is because the X-rays produced by synchrotron sources are linearly polarized in the plane of the ring. If we recall equation 2.2 , substituting in an expression for the Hamiltonian operator, we obtain the expression:

$$
\mu \propto P=\frac{2 \pi}{\hbar}\left|\left\langle\psi_{i}|\hat{\varepsilon} \cdot \vec{r}| \psi_{f}\right\rangle\right|^{2} \rho\left(E_{f}\right)
$$

where $\hat{\varepsilon}$ is the polarization vector for the electric field and $\vec{r}$ corresponds to the vector from the emitting atom to the scattering atom. For a sample with oriented bonds, such as crystallographically-aligned nanorods, the signal should depend upon the angle between the polarization and the interatomic vector $\vec{r}$. Indeed, re-formulated, the angle-dependent expression of the EXAFS signal is $[58,59]$

$$
\chi(k, \theta)=\sum_{j} 3\left\langle\cos ^{2} \theta\right\rangle \chi_{i s o}^{j}(k)=\sum_{j} \sum_{i=1}^{N_{j}} 3 \cos ^{2}\left(\theta_{i}^{j}\right) \chi_{i s o}^{j}(k)
$$


where $j$ is the number of the coordination shell (e.g. $1^{\text {st }}, 2^{\text {nd }}$, etc.), $i$ is the index for all $N_{j}$ atoms of the $j$ th shell, $\theta_{i}^{j}$ is the angle between the X-ray polarization and the interatomic vector $\vec{r}$ (pointing from the emitting atom to the $i$ th atom of the $j$ th shell), and $\chi_{i s o}^{j}(k)$ which is the isotropic contribution for an atom of the $j$ th shell (this is the signal produced from a randomly-oriented powder).

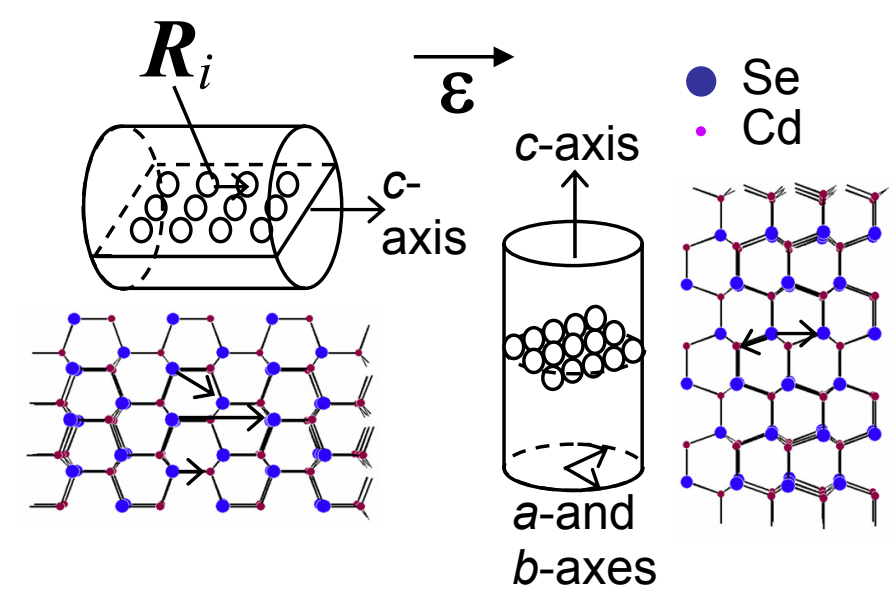

Figure 3.2. Diagram depicting preferential probing along different directions in a nanorod by polarized EXAFS. Left: When the rod $c$-axis is parallel to the beam polarization, denoted by the vector $\vec{\varepsilon}$, interatomic pairs aligned more along the $c$-axis will be probed. Right: When the rod $c$ axis is perpendicular to $\vec{\varepsilon}$, signal from the interatomic pairs perfectly parallel to the c-axis (e.g., Cd-Se pairs along the $c$-axis) is omitted.

Hence, following equation 3.2, given a crystallographically-aligned sample, different bonds in the crystal should be probed depending upon the angle $\theta_{i}^{j}$. An example of this orientation-dependent measurement is shown schematically in figure 3.2 for a CdSe nanorod. When the nanorod is oriented with its $c$-axis perpendicular to the beam polarization, the 
bonds (or interatomic pairs) aligned along the $c$-axis are not a part of the signal-only the other bonds are detected. On the other hand, when the $c$-axis is parallel to the polarization, the signal is dominated by bonds (or interatomic pairs) aligned along the $c$-axis.

\subsubsection{Angular dependencies in structural parameters of interest}

The change in the EXAFS spectra with respect to angle is known as dichroism. As is evident from equation 3.2, $\chi(k)$ is linearly related to $\left\langle\cos ^{2} \theta\right\rangle$. This angular relationship can be used to derive the angular dependence for physical parameters of interest, such as the interatomic distance $r$. The average EXAFS distance measured for a given shell and angle $\theta$ can be expressed as

$$
\bar{r}=\frac{\sum_{i}^{N} w_{i}(c) r_{i}}{\sum_{i}^{N} w_{i}(c)}
$$

where $N$ is the number of atoms in the shell, $i$ is the index atoms in the shell, $c$ is $\cos ^{2} \theta, w_{i}(c)$ is the signal weighting (a linear function of $\cos ^{2} \theta$, per equation 3.2), and $r_{i}$ is the interatomic distance between the atom being probed and its neighbor. For the CdSe wurtzite structure, which has nearly ideal tetrahedral bonding, the denominator of eqn. 3.3 remains nearly constant, no matter what the value of $\theta$ is. This means that, approximately, the measured value $\bar{r}$ is linearly proportional to $\left\langle\cos ^{2} \theta\right\rangle$. Note that this is not necessarily the case for other geometries (for example, an atom in a square planar complex with one bond perpendicular to the plane). 
For an oriented CdSe nanorod, the angular dependence of the measured value of $\bar{r}$ is expressed as

$$
\bar{r}(\theta) \cong\left(r_{\|}-r_{\perp}\right)\left\langle\cos ^{2} \theta\right\rangle+r_{\|} .
$$

Parallel and perpendicular orientations are defined with respect to the X-ray polarization and the crystallographic $c$-axis. Note that $r_{\|}$and $r_{\perp}$ refer to the averaged signal measured when the $c$-axis of the rod is parallel and perpendicular to the beam polarization. This expression is defined as an approximation because the bonding in wurtzite CdSe varies (very slightly) from being ideally tetrahedral. The quantity $r_{\|}-r_{\perp}$, which is the difference in the measured value of $r$ when the nanorod is parallel to the beam polarization and perpendicular, is defined as the dichroism. This dichroism is the actual physical quantity of interest gained from polarized EXAFS. While 3.4 is an expression for $\bar{r}$, this linear relationship applies as well to coordination numbers and Debye-Waller factors (mean squared interatomic distance distributions—a measure of disorder).

\subsection{Experimental}

\subsubsection{Crystallographically-aligned CdSe nanorod assemblies}

The liquid crystal samples used for these studies were prepared by Dr. Liang-shi Li, a former graduate student in the Alivisatos group. All samples used were very highly concentrated solutions of $3 \times 60 \mathrm{~nm}$ CdSe nanorods in cyclohexane. The nanorods had been previously washed three times to remove excess surfactant. These solutions, due to their high viscosity, were centrifuged under argon into $0.3 \mathrm{~mm}$ ID glass X-ray capillaries, 
which were flame-sealed. Initial attempts to measure EXAFS from nanorods smeared onto glass and mylar were unsuccessful, as the nanorods experienced chemical changes under the X-ray beam, evidenced in the EXAFS spectra. (Probably these changes were due to oxidation; it was difficult to effectively seal out air from these samples.) The capillary samples were very homogeneous and provided a strong signal without overabsorption. (For a review of overabsorption and other EXAFS artifacts, please see reference [60].)

\subsubsection{Bulk standards for EXAFS}

EXAFS from bulk standards of CdSe, as well as other species most likely to be present $[61,62]\left(\mathrm{CdSeO}_{3}, \mathrm{SeO}_{2}\right.$, and Se-tributylphosphine (Se-TBP)) was measured. The oxides, which are possible surface oxidation species [61, 62], were simply very finely ground powders on Kapton tape. The CdSe standard used was a nanorod solution that had been sintered at $700^{\circ} \mathrm{C}$ on graphite under argon. XRD did not show any signs of oxidation or nanoscale structures after sintering. (Other bulk CdSe standards had proved problematic due to overabsorption.) The Se-TBP, which is the Se precursor used in nanorod synthesis, was centrifuged into a glass X-ray capillary under argon and flame-sealed.

\subsubsection{Spectroscopy: data collection}

A schematic of the experiment, performed at ALS beamline 10.3.2, is shown in figure 3.3, and the sample holder for the capillary is shown in figure 3.4. This setup has special features that make it ideal for studying individual domains in the nanorod liquid crystals. First, the beamline has a microbeam, with a maximum spot size of $16 \mu \mathrm{m} \times 7 \mu \mathrm{m}$, which allows for measurements on single aligned domains of the liquid crystal. 


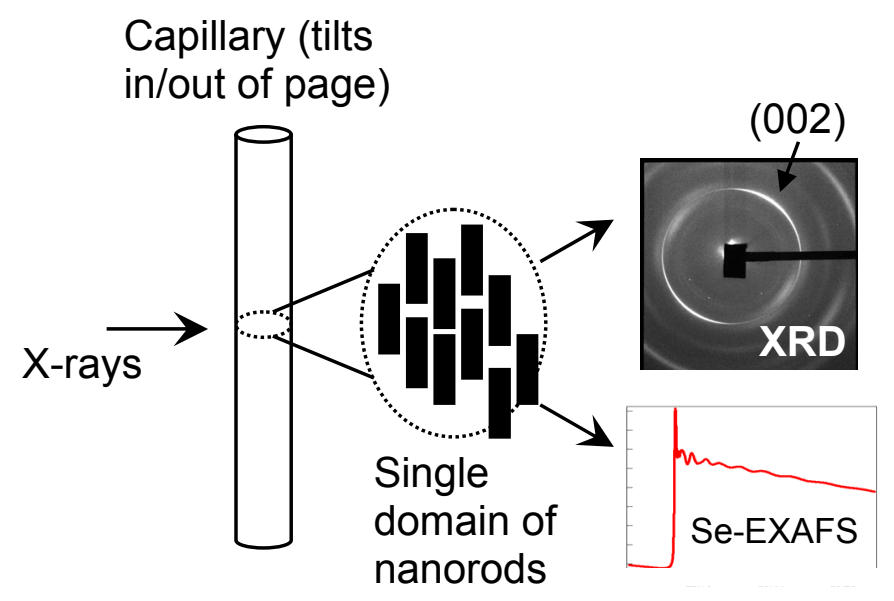

Figure 3.3. Schematic drawing of the experimental setup, from which both XRD and EXAFS data were collected from a single domain of oriented nanorods. To alter the angular alignment of the rods, the capillary was tilted into or out of the plane of this page.

Furthermore, the beamline is equipped with a CCD for powder XRD, which was used to measure the orientation of the nanorods before and after each set of scans. Finally, the beamline has a cryo-stream cooler, which was necessary to keep the liquid crystal from flowing and hence disrupting the orientation. (Originally, the experiments were performed at room temperature, but XRD measurements showed that the nanorods changed orientation during data acquisition.) The liquid crystals were cooled to $-50^{\circ} \mathrm{C}$. Due to the setup geometry, fluorescence could not be measured (the sample holder geometry obstructed the path from the sample to the fluorescence detector, which was fixed in place). Nevertheless, it is unlikely that there were measurement artifacts such as overabsorption, which would have been easily detected in the transmission data or "hole effects" (absorption 
amplitude artifacts from a sample of uneven thickness), due to the uniformity of the sample. (More information about experimental artifacts can be found in [60].)

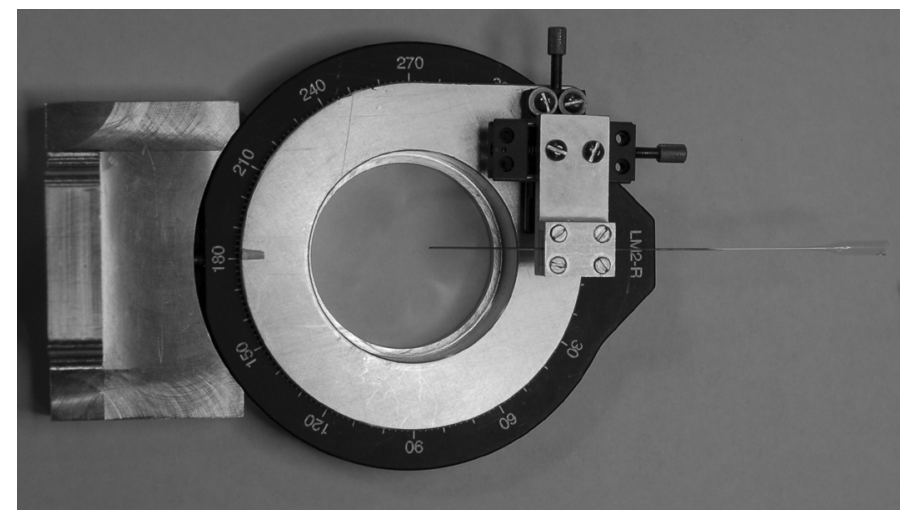

Figure 3.4. Sample holder for capillary (shown with capillary). The inner metal ring could be rotated to change the angular orientation of the capillary. The X-ray beam was approximately centered in the hole of the inner metal ring. Photograph courtesy of Dr. Matthew Marcus.

The number of orientations measured (15) was limited due to time. Each scan took $\sim 40$ minutes, and to ensure excellent signal/noise, $\sim 7$ scans per position were collected. As a control experiment, EXAFS was measured at multiple angles (11) from isotropic samples of $3 \times 60 \mathrm{~nm}$ CdSe rods, prepared in the same fashion as the liquid crystalline rods. This control was necessary to rule out any angle-dependent artifacts.

Due to the energy range available at the ALS, Cd K-edge EXAFS was not possible. Cd L-edge EXAFS measurements proved to have extremely poor signal. 


\subsection{Analysis}

\subsubsection{Data reduction/Fourier filtering}

Spectra for each angular orientation were averaged, and background subtraction and Fourier filtering performed as described in chapter 2. To obtain second and third shell data, the residuals from the first shell filtering (that is, what was left of the signal after the first shell components were subtracted) were filtered together in one window. Note that for each angle, special care was taken to reproduce the same data processing procedure (e.g., background subtraction, $E_{0}$ positioning, etc.).

\subsubsection{Additional data fitting procedures}

First shell $f_{j}\left(k_{e}\right)$ and $\delta_{j}(k)$ were obtained using experimental data from bulk CdSe. The second and third shell $f_{j}(k)$ and $\delta_{j}(k)$ functions were obtained using a mixture of bulk elemental Se data and FEFF 8 [38]. The second and third shell data was convolved (the shells could not be filtered out separately). Thus, this convolved data was fit simultaneously with second and third-shell phases and amplitudes obtained both experimentally and via FEFF calculations. (Dr. Matthew Marcus measured the elemental Se EXAFS and performed the necessary FEFF calculations).

Fitting was used to derive two major pieces of information: first, the physical parameters (interatomic distances, coordination) of the nanorods, and second, the dichroism (angular dependence) of any of these parameters. Two fitting methods were used. First, spectra taken at each angle were individually fit to equation 2.5 to extract structural 
parameters. Then these parameters were entered into a linear fit as functions of $\left\langle\cos ^{2} \theta\right\rangle$, per equation 3.4. The slope obtained from each fit is the dichroism for a given parameter.

The second type of fitting used was slightly different from that described in chapter 2. The main difference was that instead of only fitting one averaged spectrum at a time, spectra from all different angles were fit simultaneously. This offered the major benefit of increasing the degrees of freedom relative to the parameters being fit. For the fit, the residuals being minimized were

$$
\sum_{i, k}\left\{\chi_{i}(k)-\left[\left(1-\left\langle\cos ^{2} \theta\right\rangle\right) \chi_{f i t}\left(r_{\perp}, b_{\perp}, \sigma_{\perp}^{2}, \Delta E_{0, \perp}, k\right)+\left\langle\cos ^{2} \theta\right\rangle \chi_{f i t}\left(r_{\|}, b_{\|}, \sigma_{\|}^{2}, \Delta E_{0, \|}, k\right)\right]\right\}^{2},
$$

where $\chi_{i}(k)$ is the EXAFS spectrum taken at a given angle $\theta, \sigma^{2}$ is a Debye-Waller factor (the mean squared distance distributions, a measure of disorder), $b$ is the ratio of the sample coordination number to that of the bulk, and $\Delta E_{0}$ is a shift in the value for $E_{0}$. The code used to do this fitting was written in Labview 6.0 by Dr. Matthew Marcus. Assuming Gaussian distributions for the derived parameters, $1 \sigma$ uncertainties were calculated according to previously reported methods [63].

In addition to assuming a Gaussian distribution of interatomic distances for the data fitting, an asymmetric distribution of bond distances (Gaussian-broadened exponential tail function) was also used for fitting. Including this asymmetry did not yield a consistent improvement of the fit. This may stem from the use of room temperature reference data for bulk CdSe, which has already been shown to contain anharmonicity effects (with respect to 18 K data) [54]. 


\subsection{Results}

\subsubsection{General orientation-averaged structural parameters}

General structural parameters for the nanorods listed in table 3.1 were derived using the consensus fit on the aforementioned isotropic nanorod samples. While the orientationaveraged nanorod interatomic distances are not significantly different from those in the bulk, the coordination numbers are reduced. Bulk distances listed were adjusted for the experimental temperature [64].

Absolute values for $\sigma^{2}$ are not included due to the fact that the bulk CdSe standard and the nanorods were at different temperatures. In the absence of an extremely good model for the heat capacity of CdSe nanorods, it is impossible to deduce anything regarding the relative bond disorder (or vibrational properties) in the nanorods with respect to bulk.

Cd-Se $(1 \mathrm{NN})$ and Se-Se $(2 \mathrm{NN})$ were the only atomic pairs observed; no signal was found for other likely chemical species (see Fig. 3.5). Cd L-edge XANES on unaligned nanorods (prepared in a different fashion outlined in chapter 4, figure 4.3) indicated the presence of a $\mathrm{Cd}-\mathrm{O}$ species from species either like $\mathrm{CdSeO}_{3}$ or $\mathrm{Cd}_{3}\left(\mathrm{PO}_{4}\right)_{2}$ (a surrogate standard for Cd-phosphonic acid complexes). 
Table 3.1. General structural parameters for bulk and nano CdSe (orientationaveraged)

\begin{tabular}{lllll}
\hline & $1^{\text {st }}$ shell bulk & $\begin{array}{l}2^{\text {nd }} \text { shell } \\
\text { bulk }\end{array}$ & $\begin{array}{l}1^{\text {st }} \text { shell } \\
\text { nanorod }\end{array}$ & $\begin{array}{l}2^{\text {nd }} \text { shell } \\
\text { nanorod }\end{array}$ \\
\hline $\mathrm{R}(\AA)$ & 2.630 & 4.295 & $\begin{array}{l}2.625(+0.002, \\
-0.003)\end{array}$ & $\begin{array}{l}4.297 \\
( \pm 0.005)\end{array}$ \\
& & & & \\
& & 12 & $3.60( \pm 0.05)$ & 9.98 \\
$\begin{array}{l}\text { Coordination } \\
\text { number }\end{array}$ & 4 & & & $(+0.96,-$ \\
& & & & $0.72)$ \\
\hline
\end{tabular}




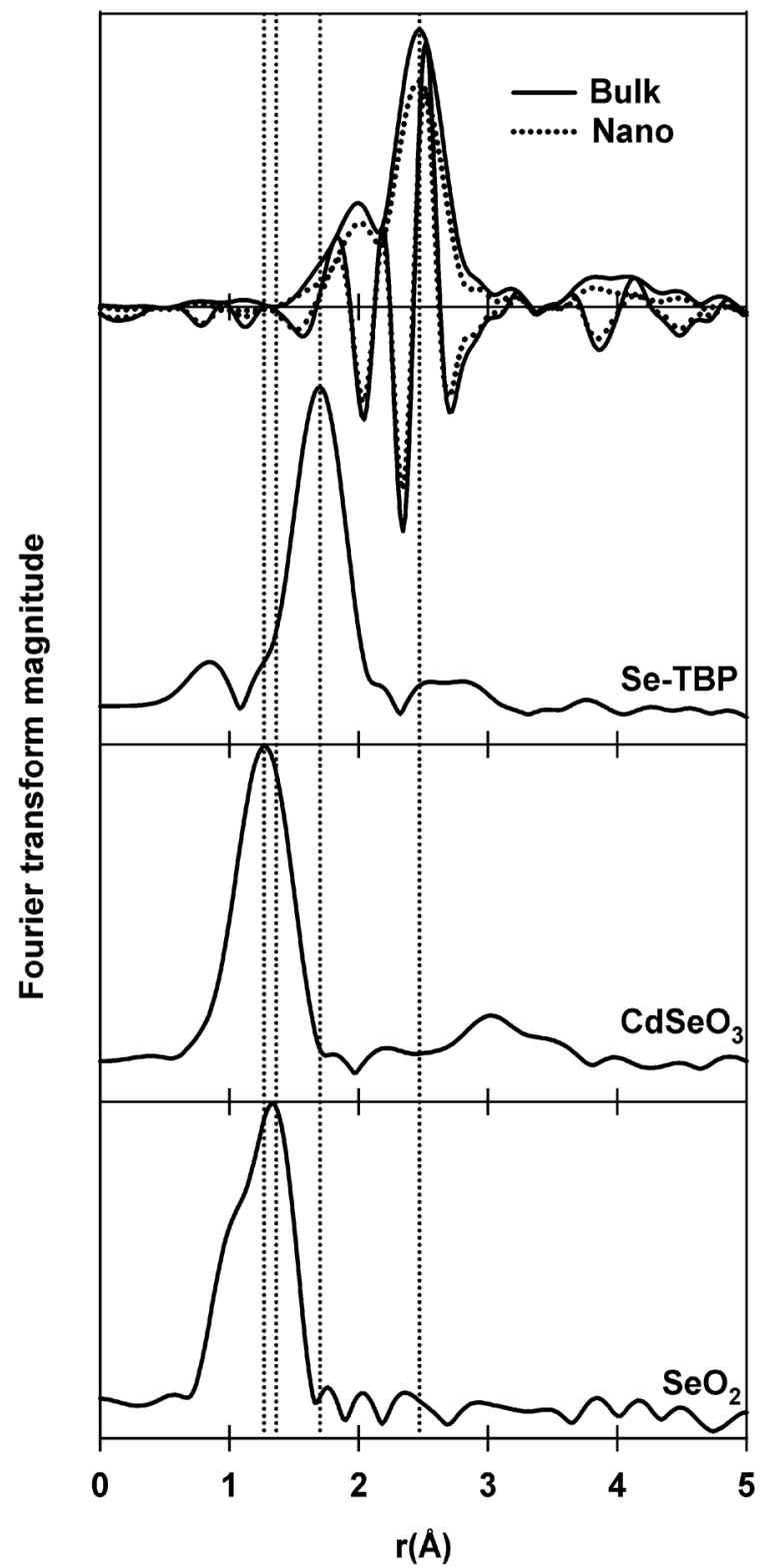

Figure 3.5. Experimental pair distribution functions of bulk and nanorod CdSe, as well as likely surface oxides and selenium-tributylphosphine (Se-TBP), a synthetic precursor. Imaginary components of the Fourier transformed data are displayed along with the magnitudes for bulk and nanorod CdSe. 


\subsubsection{Dichroisms for structural parameters}

The dichroism values are listed in tables 3.2 and 3.3. Note that for the bulk values, the term "aligned sample" refers to the averaged signal that EXAFS would measure (or "see") from an oriented crystal; it does not refer to the individual values of the axial or equatorial interatomic distances.

The only parameter displaying a significant dichroism was that of the $2^{\text {nd }}$ shell distances; the distances along the $c$-axis (axial) were smaller than those along the $a$ - $/ b$-axes (equatorial). The $2 \mathrm{NN}$ dichroism is larger than that of the bulk. As for the $1 \mathrm{NN}$, the aligned sample does not display the same dichroism present in the bulk. Results from the individual fit method were consistent with those from the consensus fit. No significant dichroism was observed for the coordination number or the Debye-Waller factors.

Table 3.2. $1^{\text {st }}$ shell dichroisms

\begin{tabular}{lllll}
\hline & $\begin{array}{c}r_{\perp}-r_{\|}(\AA), \\
b u l k\end{array}$ & $r_{\perp}-r_{\|}(\AA)$, nano & $C N_{\perp}-C N_{\|}$ & $\begin{array}{l}\sigma_{\perp}^{2}-\sigma_{\|}^{2}\left(10^{-4}\right. \\
\left.\AA^{2}\right)\end{array}$ \\
& & & & $0.55(+1.45,-$ \\
Aligned & 0.004 & $0.000(+0.001,-$ & $0.044(+0.076,-$ & $1.55)$ \\
sample & & $0.002)$ & $0.080)$ & $-0.41(-6.41,-$ \\
Isotropic & 0 & $0.000(+0.001,-$ & $-0.332(+0.368,-$ & $6.59)$ \\
& & $0.001)$ & $0.4322)$ & \\
\hline
\end{tabular}


Table 3.3. 2nd shell dichroisms

\begin{tabular}{lllll}
\hline & $\begin{array}{l}r_{\perp}-r_{\|}(\AA), \\
\text { bulk }\end{array}$ & $r_{\perp}-r_{\|}(\AA)$ & $C N_{\perp}-C N_{\|}$ & $\begin{array}{l}\sigma_{\perp}^{2}-\sigma_{\|}^{2}\left(10^{-4}\right. \\
\left.\AA^{2}\right)\end{array}$ \\
\hline $\begin{array}{llll}\text { Aligned } \\
\text { sample }\end{array}$ & -0.002 & $-0.014(+0.008,-$ & $0.044(+0.076,-$ & $0.55(+1.45,-$ \\
Isotropic & 0 & $0.006)$ & $0.080)$ & $1.55)$ \\
& & $-0.001(+0.006,-$ & $-0.332(+0.368$, & $-0.41(-6.41,-$ \\
& & $0.008)$ & $-0.432)$ & $6.59)$ \\
\hline
\end{tabular}

\subsection{Discussion}

\subsubsection{Dichroism in second-shell (Se-Se) distances}

To understand the structural origin of the measured differences between the $1 \mathrm{NN}$ and 2NN dichroisms of bulk and nanorod CdSe a collaboration was initiated with Professor Giulia Galli (University of California, Davis), Professor François Gygi (UC Davis), and Dr. Andrew Williamson (staff scientist, Lawrence Livermore National Laboratory, Livermore, CA), who performed a series of first principles, density functional theory (DFT) electronic structure calculations on approximate models of CdSe nanorods. A complete $3 \times 60 \mathrm{~nm}$ CdSe nanorod contains approximately 16,000 atoms, which was a prohibitively expensive number of atoms to simulate from first principles. Instead, the nanorods were modeled as infinitely long nanowires which are periodically repeated along the [0001] direction (long axis). The structural model was a $2.4 \mathrm{~nm}$ diameter nanowire, with (1000) and (1-100) side facets; the top view is shown in figure $3.6 \mathrm{~A}$. (A $3 \mathrm{~nm}$ diameter nanowire had too many atoms for such a calculation.) The electronic structure of the nanowire and a reference bulk structure were calculated using the Qbox [65] and ABINIT [66] ab-initio molecular dynamics 
and pseudopotential DFT codes, respectively. The wavefunctions were expanded in a plane wave basis, with a 35 Ry energy cutoff with $4 k$-points along the [0001] direction in the nanowire. Norm conserving, Troullier-Martins pseudopotentials, with $4 \mathrm{~d}^{10} 5 \mathrm{~s}^{2}$ and $4 \mathrm{~s}^{2} 4 \mathrm{p}^{4}$ valence electrons were used to represent the $\mathrm{Cd}$ and Se cores, respectively. For both the bulk and nanowire systems the atomic coordinates were relaxed to their closest, lowest energy atomic positions. The dimensions of the simulation supercells were also relaxed to their lowest energy configurations to allow for changes in the structural parameters $c, u$ and $a$.
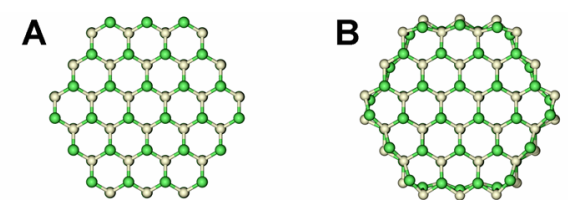

FIG. 3.6. Top views of unrelaxed (A) and relaxed (B) $2.4 \mathrm{~nm}$ diameter CdSe nanowire from DFT calculations. Green atoms are $\mathrm{Cd}$, white atoms are Se. The core atomic positions are mostly unchanged, while the outer atoms shift.

The ideal and relaxed nanowire structures are shown in Fig. $3.6 \mathrm{~A}$ and B. The core of the nanowire showed relatively minor structural relaxations from the ideal bulk wurtzite structure, while the surface of the nanowire spontaneously reconstructed to lower its energy. In particular, surface atoms with a coordination number of 2 experienced the most dramatic structural relaxations, wherein Se-Cd U-shaped structures rotate into the surface of the wire to increase their coordination. Similar surface reconstructions have also been predicted for CdSe quantum dots [67]. After reconstruction, the nanowire exhibited a broad distribution 
of bond lengths. The Cd-Se bonds in the core of the nanowire deviated from their bulk values by $<1 \%$, while those on the surface were altered by up to $5 \%$.

To compare the DFT-predicted relaxed structures with the structures measured by EXAFS, in Fig. 3.7 D and E the 1NN and 2NN dichroism of the DFT relaxed structures is plotted. The qualitative changes in the dichroism of both the $1 \mathrm{NN}$ and $2 \mathrm{NN}$ bonds are similar to those measured by EXAFS (Fig. $3.7 \mathrm{~A}$ and B). For the $1 \mathrm{NN}$, the relaxed bulk structure (dotted line) exhibits a positive dichroism (elongation) along the $c$-axis, while the relaxed nanowire (solid black line) exhibits almost no dichroism, as was observed with EXAFS. For the $2 \mathrm{NN}$ the bulk structure exhibits a small elongation along the $c$-axis, while the nanowire exhibits a strong negative dichroism, with smaller 2NN bond lengths along the c-axis, again in agreement with EXAFS measurements.

In addition to predicting the total change in dichroism when moving from a bulk to nanowire geometry, the atomistic simulations can investigate the structural origin of the dichroism. In figures $3.7 \mathrm{D}$ and $\mathrm{E}$, the contributions to $1 \mathrm{NN}$ and $2 \mathrm{NN}$ dichroism from atoms in the core (dashed line) and on the surface (dotted and dashed line) of the nanowires are separated. This analysis shows that the core $1 \mathrm{NN}$ bonds expand by $\sim 1 \%$ compared to the bulk and show a small positive dichroism, while the surface bonds contract by $\sim 0.5 \%$ and do not show any dichroism. In contrast, both the core and surface $2 \mathrm{NN}$ Se-Se bonds show a strong negative dichroism, arising from an expansion of $2 \mathrm{NN}$ distances perpendicular to the $c$-axis.

While the calculated and measured trends in bond lengths show good agreement, the DFT predicted bond lengths are approximately $1 \%$ shorter than the measured ones. This 
underestimate of the CdSe bulk bond length has been observed in previous calculations [68] and results from known "over-binding" in semiconductors and insulators when described within the local density approximation of DFT. It is notable that the measured and predicted structural dichroisms qualitatively agree, even though no surfactant molecules were included in the DFT simulations. This result supports previous theoretical predictions [67], indicating that the interaction between surfaces of small CdSe dots and organic surfactants is weak enough not to significantly perturb surface reconstructions obtained for bare dots. Previous calculations on bulk CdSe surfaces indicated that surfactants will reduce surface reconstructions [19], which may explain why the DFT calculations presented here predict a larger 2NN dichroism than measured.

The observed compression of CdSe nanorods along their $c$-axes appears to depend not only on their size, but their shape. II-VI quantum dots of comparable size have displayed decreased bond distances with respect to the bulk [69]. A study on CdSe nanodots showed that both the $c$ - and $a$-axes were compressed [70], rather than just the $c$-axis. Bulk CdSe mechanical properties do not explain the anisotropic compression in the nanorods, as the elastic modulus along the $c$-axis $(87.1 \mathrm{GPa})$ is larger than that along the $a$-axis $(74.6 \mathrm{GPa})$ [71]. As neither size nor bulk properties explain the preferential $c$-axis compression, the origin of this compression must lie in the rod shape. This is consistent with structural studies on other anisotropic nanomaterials, such as colloidal core-shell CdSe-ZnS nanorods. As the ZnS shell thickness was increased, XRD revealed an overall compression which was maximal along the $c$-axis [72]. Wurtzite $\mathrm{GaN}$ and $\mathrm{ZnO}$ nanowires [73, 74] also displayed 

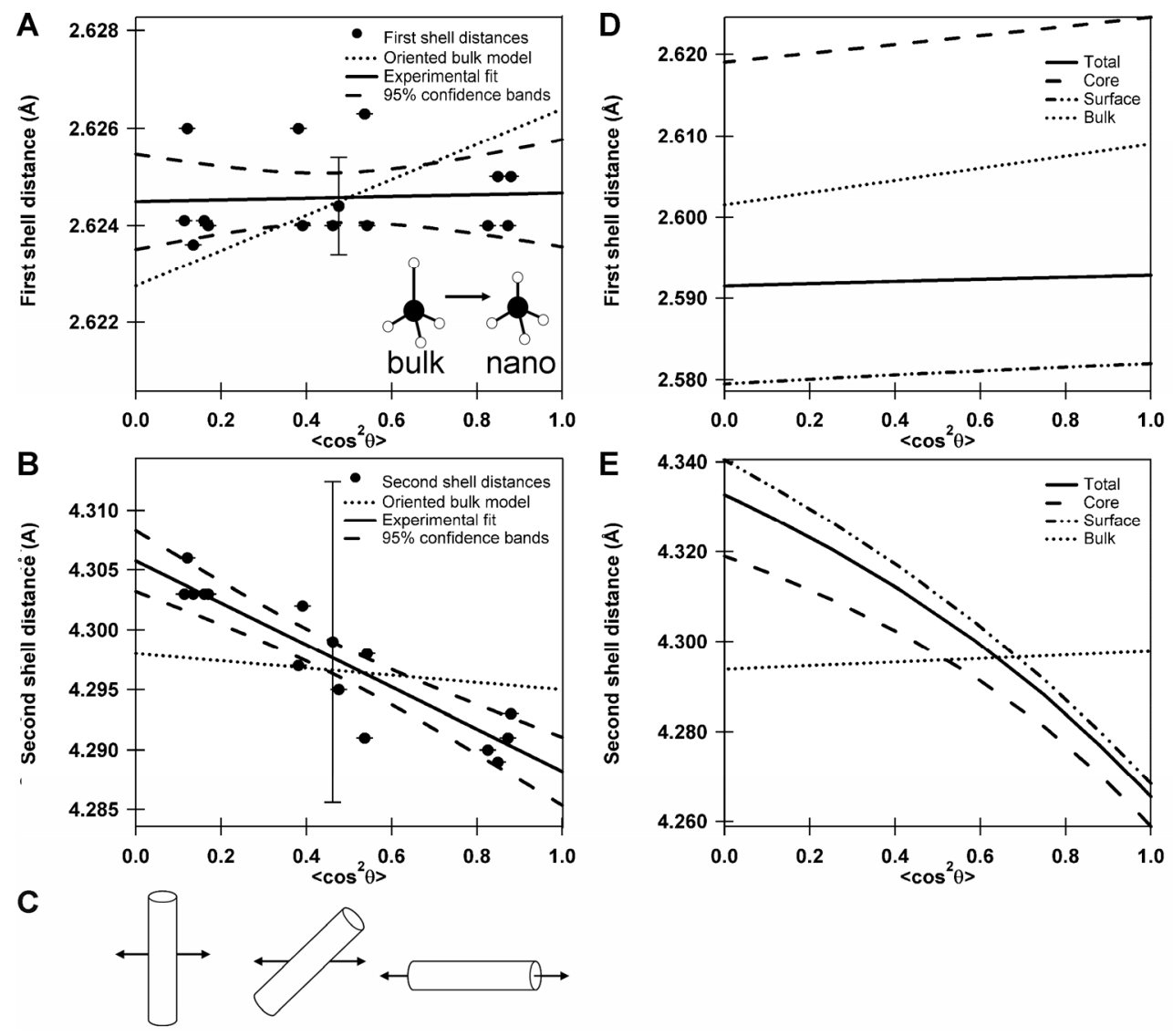

FIG. 3.7. Cd-Se distances (first shell) versus $\left\langle\cos ^{2} \theta\right\rangle$. Inset: schematic drawing of $\mathrm{SeCd}_{4}$ tetrahedron in nanorods and bulk showing loss of distortion in nanorods. B. Se-Se distances (second shell) versus $\left\langle\cos ^{2} \theta\right\rangle$. For both $\mathrm{A}$ and $\mathrm{B}$, the dashed curves denote the $95 \%$ confidence bands, while the dotted lines are calculated for an oriented ideal bulk crystal of CdSe. The error bars displayed in each of these plots are representative of the systematic error in each distance measurement. C. Schematic of nanorod orientation with respect to the X-ray polarization, denoted by the double-headed arrow. D. Plot of $\cos ^{2} \theta$-weighted Cd-Se distances from DFT calculation of a relaxed nanorod versus $\cos ^{2} \theta$. E. Plot of $\cos ^{2} \theta$-weighted Se-Se 
distances from DFT calculation of a relaxed nanorod versus $\cos ^{2} \theta$. The dots-and-dash line denotes the surface nanorod distances, the dashed lines are the core nanorod distances, and the solid line the average of all distances. The dotted lines are bulk CdSe distances derived from the DFT calculation. In A and E the oriented bulk model data are shifted downwards by $0.007 \AA$ and upwards by $0.04 \AA$, respectively, for a better visual comparison. 
asymmetric changes in atomic distances, although their dichroism was of the opposite sign of the one observed here (their long axes stretched, while their short axes shrank). Bulk $\mathrm{ZnO}$ deviates more from perfect tetrahedral bonding than nanowire $\mathrm{ZnO}$ [74], similar to what occurs in $\mathrm{CdSe}$. When comparing the behavior of $\mathrm{ZnO}$ and $\mathrm{GaN}$ nanowires with that of CdSe rods, it is important to note that both of these nanowire materials were grown differently than the CdSe nanorods with no surfactants.

\subsubsection{Significance of nanorod coordination numbers}

The $\mathrm{CN}$ of the Se atoms in the nanorods was also investigated to probe the $\mathrm{Cd}$ content on the nanorod surfaces. The $1 \mathrm{NN}$ and $2 \mathrm{NN}$ CNs measured for the nanorods are $3.6( \pm 0.05)$ and $9.98(+0.96,-0.72)$, respectively. These $\mathrm{CNs}$ fall in between those expected for a completely Cd-terminated wire $(4,9.94)$ and those expected for a stoichiometric wire (3.4, 10.1), indicating, as expected, a slightly Cd-rich surface. These results are consistent with Rutherford backscattering spectroscopy performed on CdSe dots synthesized in a similar manner [75]. Additionally, no angular dependence of the $1 \mathrm{NN}$ and $2 \mathrm{NN} C \mathrm{CN}$ in the nanorods is observed. This is consistent with nanorod models based on the ideal wurtzite lattice structure, which predict that the angular dependence averages to zero when the contributions from all surface facets of a cylindrical rod are included.

As the surface is not entirely Cd-coated, the possibility of having other atoms bound to Se was explored. Data fits did not improve when including the shells from reference compounds of likely surface species, indicating that the only atom bound to Se is Cd [6]. Thus, there is probably unpassivated Se on the nanorod surfaces, which agrees with previous 
theoretical and experimental work on quantum dots $[19,76]$. The only other way to produce a reduced $\mathrm{Se} \mathrm{CN}$ would be the presence of $\mathrm{Cd}$ vacancies. This seems unlikely as $2.5 \%$ of the $\mathrm{Cd}$ in the particles would have to be removed, a rather high percentage.

\subsection{Potential caveat: Presence of two first-shell and second-shell distances in bulk standard}

The analysis relied upon fitting to amplitude and phase functions derived from empirical measurements of bulk CdSe. A potential problem with this analysis is that for bulk CdSe, a single average distance was assumed, rather than the two different distances that are actually present. The bonds along the $c$-axis are $0.005 \AA$ longer than the rest of the bonds. There are two different Se-Se distances as well; those along the $c$-axis are shorter. Here, it is shown that the effect of having two different distances present (for first and second shell distances) is insignificant and hence the assumption of a single distance is valid.

There are two distances present for both the first shell and second shell in CdSe. The distribution of these distances can be described as the following:

$$
P(r)=x \delta\left(r-r_{1}\right)+(1-x) \delta\left(r-r_{2}\right)
$$

For $1 \mathrm{NN}$ distances, $x=0.75$, and for $2 \mathrm{NN}$ distances, $x=0.5$.

With a standard cumulant expansion $[77,78]$, the EXAFS signal can be expressed as

$$
\chi(k)=\operatorname{Im}\left[A(k) e^{2 i k\langle r\rangle-2 k^{2} \sigma^{2}-\frac{4}{3} i k^{3} C_{3}+\ldots}\right] .
$$


Hence, a distribution of bonds in the standard will affect the anharmonicity term $C_{3}$. As $C_{3}=\left\langle\left(r-\langle r\rangle^{3}\right)\right\rangle$, using the distribution $P(r)$ given in equation 3.6, we find

$$
C_{3}=x(1-x)(1-2 x)\left(r_{1}-r_{2}\right)^{3},
$$

which, for $x=3 / 4$ and a bond distance difference of $0.005 \AA$, is $-1.17 \times 10^{-8} \AA$. The contribution of the bond distance distribution to anharmonicity is much smaller than previously measured values for bulk cadmium selenide; $C_{3}$ was measured at $250 \mathrm{~K}$ (with 18 $\mathrm{K}$ data as a reference) to be $\sim 8 \times 10^{-5} \AA^{3}[54]$. For the $2 \mathrm{NN}$ distances, $x=1 / 2$, which makes $C_{3}=0$. It can therefore be concluded that the assumption of an averaged bond value for the CdSe standard is valid.

\subsection{Conclusions and outlook}

In conclusion, a combination of EXAFS measurements and DFT calculations was used to characterize the structure and surfaces of CdSe nanorods. In the rods, $1 \mathrm{NN}$ and 2NN distances are reduced along the $c$-axis with respect to bulk CdSe, while distances along the $a / b$-axes are mostly unchanged. Density functional theory predictions for the relaxed structures qualitatively agree with these measurements. In addition, the calculations indicate that the most significant structural changes accounting for the measured dichroism occur on the surface of the nanowires. Further comparisons between nanowire models and the measurements show that the nanorod surfaces are Cd-rich, with some unpassivated Se atoms. 
This investigation shows that EXAFS measurements, combined with ab-initio calculations, represent a powerful tool to investigate surface reconstructions at the nanoscale.

Future investigations of semiconductor nanocrystal surfaces, including CdSe nanoparticles, could hopefully include Cd K-edge EXAFS, as well as some soft X-ray spectroscopy on phosphorous or oxygen to better probe the attachment geometry of the surfactants. Ultimately, if shorter nanorods could also be aligned, with a higher fraction of rod tips versus sides, perhaps more information could be derived regarding the structure and composition of the tips versus the sides, in conjunction with other methods such as X-ray photoelectron spectroscopy. Hopefully, this basic process of using crystallographicallyaligned assemblies for orientation-specific measurements could be applied to other shapes of nanocrystals. 


\section{CHAPTER 4. X-RAY ABSORPTION SPECTROSCOPY OF CDSE/ZNS CORE-SHELL NANORODS}

\subsection{Motivation and background}

As previously mentioned, the size- and shape-tunable optical properties of cadmium selenide nanorods have spurred interest in their use for various applications such as lightemitting diodes or orientation-specific fluorescence bioprobes [48][79]. Unfortunately, CdSe nanorods are at a disadvantage due to their weak fluorescence, with a quantum yield $<1 \%$ [72]. When a CdSe nanorod absorbs a photon of adequate energy, an electron is excited up to the conduction band, and a hole is created in the valence band. This electron-hole pair is called an exciton. Fluorescence will only occur if the electron relaxes back down into the valence band; in other words, the electron and hole must recombine. If intraband energy states, commonly formed due to the presence of surface defects or unpassivated bonds, are present, electrons or holes may be "trapped" in these states, thus preventing exciton recombination [11].

Given the detrimental effect of the surface upon nanorod fluorescence yield, previous members of the Alivisatos group developed a protocol for surface modification [72]. Specficially, they epitaxially grew shells of higher band-gap materials ( $\mathrm{ZnS} / \mathrm{CdS}$ ) onto the surfaces of CdSe nanorods. This type of surface modification had already been demonstrated to work for zero-dimensional CdSe nanocrystals (spherical "quantum dots") [80]. Growing epitaxial shells increases fluorescence yield in two ways. First, as the shell is a 
higher band-gap material than the core, the excitons are energetically more confined to the core, thus increasing their probability of recombination (and hence the probability of fluorescence). Second, by passivating unbonded atoms on the core, surface states are eliminated that would otherwise "trap" charge carriers.

In developing the synthesis protocol for epitaxial shell growth on nanorods, two primary discoveries were made. First, it was found that epitaxy in the higher-dimensional (pseudo 1D) nanorods was not the same as it was for the $0 \mathrm{D}$ quantum dots. In the dot synthesis, a $\mathrm{ZnS}$ shell is directly grown onto the CdSe dot. For nanorods, this was not possible; the shell stock solution not only had $\mathrm{Zn}$ and $\mathrm{S}$ precursors, but a Cd precursor as well. It is thought that the epitaxial strain of growing $\mathrm{ZnS}$ directly onto $\mathrm{CdSe}$ is too energetically unfavorable for the nanorod geometry. Second, it was found that exposing core-shell nanorods to intense visible light effected a significant and irreversible increase in quantum yield. It was posited that this phenomenon arose from structural rearrangements at the core-shell interface or on the surface; hence the process was dubbed "photoannealing". Such observations naturally inspire curiosity regarding the atomic structure of the surfaces and interfaces present in core-shell nanorods, both to elucidate the mechanics of epitaxy in higher-dimensional nanostructures, as well as the origins of interesting optical behavior. This chapter concerns initial studies upon surface and interface structure in higher-dimensional core-shell nanocrystals, specifically CdSe-ZnS core-shell nanorods. It is hypothesized that there are two major factors influencing the core and shell structures of the nanorods: the large surface area-to-volume ratio, and epitaxial strain. The presence of the surface may induce bond rearrangements or relaxations to minimize surface energy; epitaxial 
strain might cause the core and shell lattices to contract or expand to minimize strain energy. Shape-induced effects, such as anisotropic bond contractions, are anticipated as well.

Core and core-shell nanorod structures were examined with X-ray diffraction (XRD) and extended X-ray absorption fine structure (EXAFS) spectroscopy. EXAFS was particularly beneficial for this experiment due to its elemental specificity, which allowed the core to be probed separately from the shell. As with the plain CdSe nanorods, EXAFS was also useful due to being a local probe (and hence sensitive to surface or interfacial bonding).

\subsection{Experimental}

\subsubsection{Preparation of the nanorods}

\subsubsection{Synthesis}

Synthesis of the CdSe/ZnS core-shell nanorods has been described previously [72] and is only briefly discussed here. Nanorods were synthesized by the late Dr. Benjamin Boussert. They were characterized with TEM, XRD, and UV-visible absorption/emission spectroscopy by both Dr. Boussert and D. Aruguete. CdSe nanorods were synthesized as described in chapter 3, except that the prolonged growth steps via slow injection were eliminated, resulting in shorter CdSe nanorods $(3.5( \pm 0.3) \mathrm{nm} \times 16.8( \pm 2.0) \mathrm{nm})$. After washing with methanol to remove excess surfactant, the nanorods were dissolved in chloroform, which was mixed with heated, de-gassed TOPO. The chloroform was removed with pumping. A mixed stock solution of dimethyl cadmium, diethyl zinc, and 
hexamethyldisilathiane was added in slowly to epitaxially grow a graded shell of CdS/ZnS on the CdSe rods (figure 4.1).

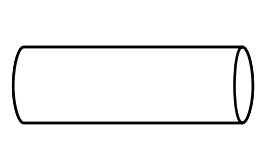

1. CdSe

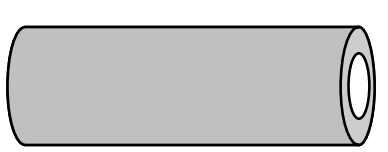

2. CdS on $\mathrm{CdSe}$

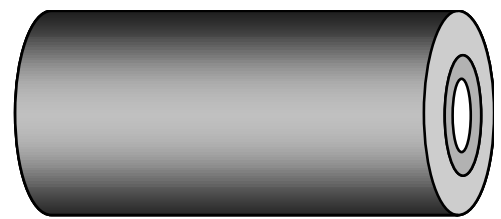

3. ZnS on CdS on CdSe

Figure 4.1. Schematic of the growth of a graded CdS/ZnS shell on a CdSe nanorod.

Previous attempts to grow a pure $\mathrm{ZnS}$ shell, rather than a graded shell, onto nanorods proved unsuccessful. An intermediate layer of CdS between the CdSe and $\mathrm{ZnS}$ is believed to relieve epitaxial strain, as the lattice parameters of $\mathrm{CdS}$ are between those of $\mathrm{CdSe}$ and $\mathrm{ZnS}$. The presence of this intermediate layer has been indirectly substantiated by energy dispersive X-ray (EDX) analyses, as well as UV-visible absorption spectroscopy. (Cation or anion exchange along the core-shell interface had not been ruled out previous to the work presented in this chapter.) As per previous results, XRD showed that the core-shell nanorods retain the wurtzite crystal structure of the cores.

Nanorods with shells of three different thicknesses were prepared, referred to in this chapter as thin-, medium-, and thick-shell nanorods. Different shell thicknesses were achieved by varying the amount of shell stock solution for growth. Thin-shell rods had dimensions of $3.9( \pm 0.5) \mathrm{nm} \times 16.4( \pm 2.4) \mathrm{nm}$, medium-shell rods had dimensions of 4.1 $( \pm 0.4) \mathrm{nm} \times 17.0( \pm 2.0) \mathrm{nm}$, and thick-shell rods had dimensions of $5.1( \pm 0.5) \mathrm{nm} \times 18.8( \pm$ 
2.3) $\mathrm{nm}$. Size distributions were measured from TEM images using samples of at least 100 nanorods. While the sample sizes may appear to have significant overlap, the UV-visible absorption spectra (figure 4.2) confirmed that their shells were definitely of different thicknesses. A notable red-shifting of the first exciton absorption feature can be observed, caused by the increase in the size of the nanocrystals [72].

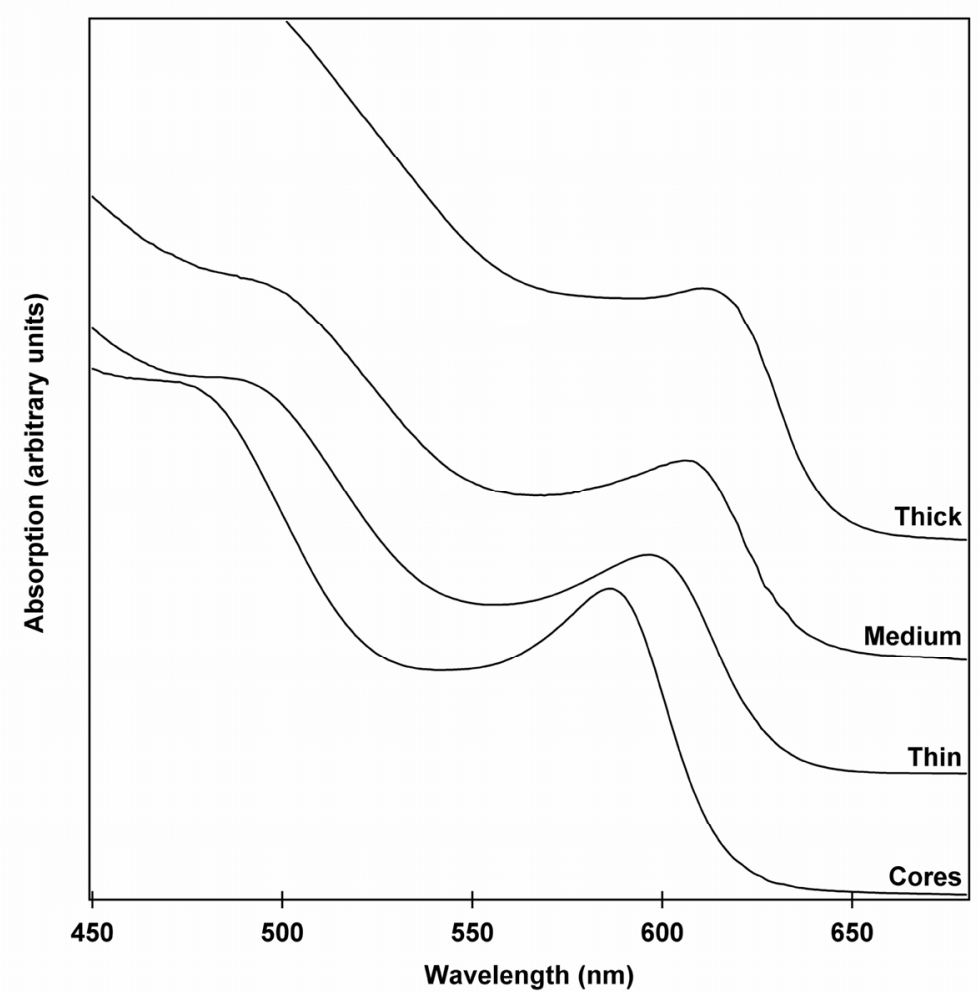

Figure 4.2. UV-visible absorption spectra from core and core-shell nanorods. The first exciton absorption feature, positioned at $\sim 590 \mathrm{~nm}$ for the cores, progressively red-shifts with increasing shell thickness. 


\subsubsection{Photoannealing of core-shell nanorod samples}

Core-shell nanorods, upon exposure to visible or UV light (under air-free conditions) experience a dramatic irreversible increase in their visible photoluminescence intensity [72]. It has been posited that this increase is due to a process known as "photoannealing", in which light induces structural rearrangements of the crystal lattice, eliminating surface or interfacial defects. Such defects can create energy states which trap charge carriers, preventing exciton recombination and hence fluorescence. Therefore, removing such defects should allow more recombination events to occur, increasing fluorescence intensity.

While both photoannealed and unannealed samples (these were kept in the dark until measurement) were both examined with X-ray absorption spectroscopy, the data presented here are only for photoannealed samples. This is because initial experiments performed by Dr. Matthew Marcus could not clearly establish whether X-ray beam was annealing the unannealed samples; considerations of the power input by the X-ray beam could not rule out this possibility.

\subsubsection{Preparation of nanorods for XAS}

All preparation, including cleaning, was conducted in an argon drybox. Cleaned nanorods were highly concentrated in toluene. The solutions were pipetted, by capillary action, into open-ended $0.3 \mathrm{~mm}$ ID X-ray capillaries of glass, borosilicate, or quartz

(produced by breaking the ends of commercially-available capillaries). The ends were sealed with Torrseal epoxy and allowed to set for three days.

While capillary samples could be used for Se and Zn K-edge EXAFS, the capillary walls were too thick for $\mathrm{Cd} \mathrm{L}_{3}$-edge or $\mathrm{S} \mathrm{K}$-edge XAS. Not only are these edges at lower X- 
ray energies $(\sim 3540 \mathrm{eV}$ and $\sim 2470 \mathrm{eV}$, respectively), but the $\mathrm{X}$-ray flux at the beamline used (ALS, 10.3.2) is reduced at low X-ray energies. Therefore, the samples were prepared on thin $\mathrm{Si}_{3} \mathrm{~N}_{4}$ X-ray substrates manufactured by Silson Ltd. (UK) per the procedure in figure 4.3.
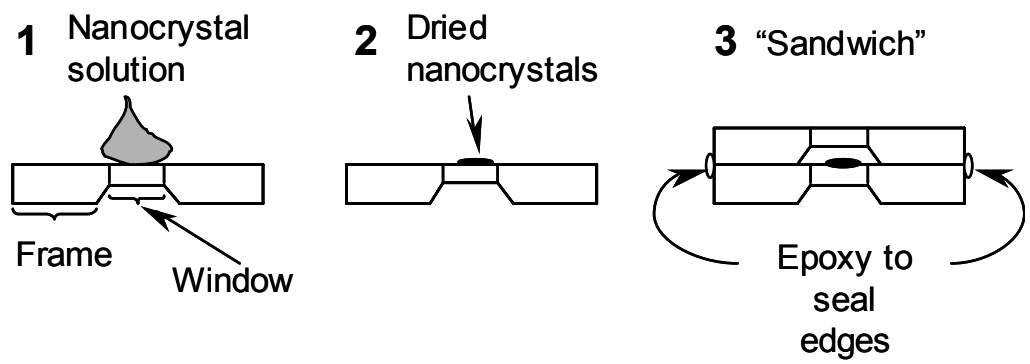

Figure 4.3. Sample preparation procedure for Cd and S XAS.

$2 \mu \mathrm{L}$ of nanocrystal solution were deposited with a pipettor onto the flat side of a 100-200 nm thick $\mathrm{Si}_{3} \mathrm{~N}_{4}$ square $\mathrm{X}$-ray window (1-5 mm edge length) and allowed to dry. The X-ray window was held with a pair of anticapillary electron microscopy (EM) tweezers and tilted during drying to ensure that the droplet remained upon the window, rather than drying onto the window frame. Additional drops of solution were added as necessary to form an opaque mass of dried nanocrystals readily visible to the naked eye.

Once a satisfactory amount of nanocrystals had been deposited, the concave side of another $\mathrm{Si}_{3} \mathrm{~N}_{4}$ window was placed on top. One side of this "sandwich" was secured with a pair of EM tweezers, and Torrseal epoxy was carefully applied to the open edges of the sandwich. After 24 hours, this procedure was repeated for the other side. The epoxy on both sides set for the maximum setting time (3 days). During epoxy setting in the drybox, 
samples were shielded from light to prevent photooxidation from any residual oxygen or water. Samples were transferred to the ALS under argon in sealed vials.

\subsubsection{Spectroscopy and diffraction}

The setup was the same as described in chapter 3, except for two main features.

First, all data was measured on room temperature samples. Second, Cd and S XAS measurements were conducted in a slightly different fashion to improve signal. Only X-ray fluorescence was collected for Cd and S XAS, as there was not enough sample for useful X-ray transmission data. The X-ray beam path was encased in helium by attaching a modified piece of pipe to the X-ray fluorescence detector, as shown in figure 4.4. This socalled "beampipe" had X-ray transparent mylar windows and was constantly purged with an He gas supply. The beampipe was necessary for reducing air

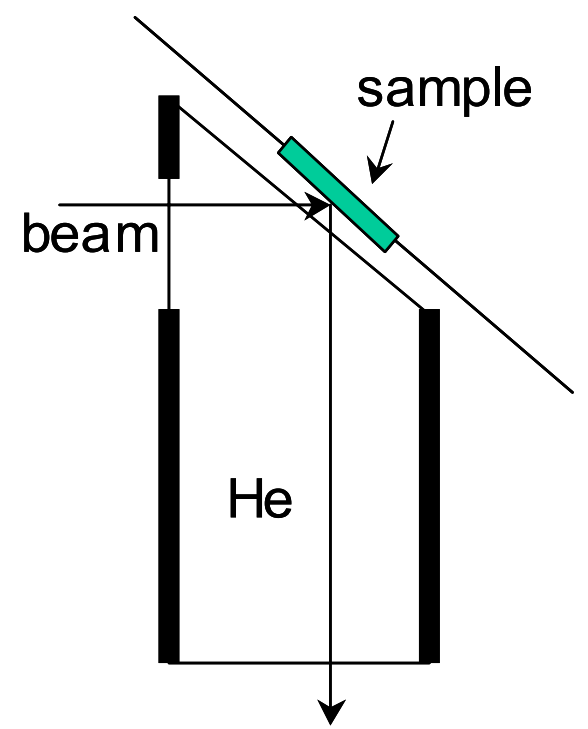

Fluorescence detector absorption of the lower energy X-rays, increasing the signal substantially. Powder XRD at $16 \mathrm{keV}$ was collected as part of the standard characterization for the nanorods, to confirm that they had undergone the same structural changes as previously documented [72]. Data was collected on a CCD and integrated using the Fit2D software package.
Figure 4.4. Helium-filled beampipe attachment. The opening on the side and the angled end of the beampipe are covered with X-ray transparent mylar. Alumina (National Institute of Standards and Technology) was used as a calibrant. 
EXAFS and XRD bulk standards used were commercially purchased, except for the bulk CdSe standard (see chapter 3) and the bulk wurtzite ZnS, provided by Dr. Benjamin Gilbert, LBNL.

\subsection{Powder X-ray diffraction results}

The powder XRD diffraction results are shown in figure 4.5 for three reflections: 100, 002, and 101. The (002) planes are perpendicular to the $c$-axis of the nanorod, while the (100) planes are parallel. The 002 reflection is considerably sharper and narrower due to the shape of the nanorod; there are many more (002) planes than there are (100) or (101) planes, hence size-broadening effects are not so pronounced.

The $d$-spacings of these planes decrease as the shell thickness increases. In particular, relative to the cores, the (002) interplanar spacings show the most compression. These results qualitatively agree with previously recorded XRD for core-shell nanorods.

Table 4.1. XRD Peak Position Changes as a Function of Shell Thickness

\begin{tabular}{lccclll}
\hline Sample & $\mathrm{q}_{(100)}$ & $q_{(002)}$ & $q_{(101)}$ & $\begin{array}{l}(100) d- \\
\text { spacing } \\
\text { change }\end{array}$ & $\begin{array}{l}(002) d- \\
\text { spacing } \\
\text { change }\end{array}$ & $\begin{array}{l}(101) d- \\
\text { spacing } \\
\text { change }\end{array}$ \\
\hline Cores & 17.07 & 18.01 & 19.08 & $0 \%$ & $0 \%$ & $0 \%$ \\
Thin shell & 17.10 & 18.16 & 19.18 & $0.20 \%$ & $0.80 \%$ & $0.54 \%$ \\
Medium shell & 17.10 & 18.22 & 19.27 & $0.20 \%$ & $1.15 \%$ & $1.00 \%$ \\
Thick shell & 17.25 & 18.34 & 19.42 & $1.10 \%$ & $1.80 \%$ & $1.75 \%$ \\
\hline
\end{tabular}




\subsection{EXAFS results and discussion}

Analysis and fitting methods used for the data were the same as those described in chapter 3. As the core-shell nanorods were not crystallographically aligned, no further analysis regarding polarization effects was necessary. All data was $k^{2}$-weighted for analysis.

In chapter 3, discussion of the EXAFS Debye-Waller factors (DWFs), otherwise known as the statistical variances in the interatomic distance distributions (assuming Gaussian bond distributions) was highly limited. This was due to the fact that the data from the EXAFS empirical standard was measured at a different temperature than the nanocrystals. The nanorod data was fit using the phase and amplitude functions derived from the empirical bulk data. Thus, in the absence of good heat capacity models for nanorods, it was not possible to draw any conclusions about bond disorder in the samples. For the core-shell studies, all samples were measured at room temperature, allowing for comparisons between the nanorod samples and the bulk standards.

By definition, the DWFs for bulk standards are zero. Thus, positive DWFs for a sample mean that the sample is more disordered than the bulk standard, whereas negative DWFs mean that the sample is more ordered.

\subsubsection{Se K-edge EXAFS}

Fourier transform magnitudes for the raw Se data of the CdSe core nanorods, the $\mathrm{CdSe} / \mathrm{ZnS}$ core-shell nanorods, and various standards are shown in figure 4.6. No peak corresponding to the $\mathrm{Zn}$-Se first shell signal is present in the nanorods, confirming that there 
is no significant diffusion of Se or $\mathrm{Zn}$ through the $\mathrm{CdSe} / \mathrm{CdS} / \mathrm{ZnS}$ interface. Of all of the Se species considered in the fitting, only CdSe was detected.

\subsubsection{Se first shell distances (Cd-Se distances)}

The Cd-Se distances are displayed in figure 4.7 A. Contrary to the trends displayed in the XRD data of section 4.3, core-shell nanorod Cd-Se distances are not significantly reduced with respect to those in the cores, except for the distances in the thick-shell nanorods. While these EXAFS results may appear to contradict with the XRD data, the differences between these two structural probes must first be considered.

One of the primary differences between EXAFS and XRD is that the EXAFS is an orientation-averaged technique (unless the sample is crystallographically aligned; see the studies in chapter 3). For the first shell, $1 / 4$ of the EXAFS signal arises from the Cd-Se bonds parallel to the $c$-axis; the other $3 / 4$ of the signal comes from those that are more aligned with the $a$-and $b$-axes. Thus, even if the Cd-Se bonds parallel to the $c$-axis of the rod are compressed, if the other Cd-Se bonds have expanded accordingly, then EXAFS will register no change in the overall Cd-Se distance. This is a possible scenario for the core-shell nanorods.

Such a hypothesis involving the expansion of the bonds along the $a$ - and $b$-axes may seem to be invalidated by XRD data concerning the (100) interplanar spacings, which are reduced with increasing shell thickness. Nevertheless, this seeming contradiction illustrates another primary difference between XRD and EXAFS. While the XRD is a convolution of diffraction from both the CdSe and the CdS/ZnS shell, EXAFS specifically probes one set 
of atoms at a time. Thus, if the core expands, but the shell contracts, it might still be possible to obtain an XRD signal showing an overall contraction.

Parsing out such hypotheses may require simulation of XRD patterns from coreshell nanorods, as well as a deeper analysis of the EXAFS Debye-Waller factors. The latter shall be discussed in the next section.

\subsubsection{Cd-Se Debye-Waller factors}

\subsection{General contributions to disorder in materials}

Disorder in EXAFS is measured as the Debye Waller factor, or the variance in the distribution of interatomic distances (assuming a Gaussian distribution). Contributions to disorder in a structure can be divided into two categories: static, and temperature-dependent [31]. Static contributions refer to distributions of interatomic distances that do not change dramatically with time or temperature. One example of this has already been alluded to in the previous section. For example, the presence of both compressive and tensile strain in a nanorod might result in two different overall bond lengths for most temperature ranges.

The presence of two bond lengths, rather than one, by definition is a broader distribution in bond lengths; this would be a static contribution to the DWF.

Temperature-dependent contributions refer to the vibrational properties of a material. For a crystal, as temperatures are raised, more vibrational modes can be sampled, which increases the overall distribution in interatomic distances sampled by EXAFS. If the vibrational modes available to a crystal are reduced, either by reducing temperature or via mechanical means, then the DWF measured from that crystal should be reduced. 


\subsection{Cd-Se DWFs}

In figure $4.7 \mathrm{~B}$, it is apparent that the DWFs decrease as the shell thickness increases. This trend can be easily explained. First, capping a plain CdSe nanorod should significantly decrease Cd-Se DWFs, because underbonded surface $\mathrm{Cd}$ and Se are eliminated. As the shell is thickened, additional strain may exert more compressive pressure upon the nanocrystal interiors, reducing the vibrational degrees of freedom, which would also cause a measurable decrease in the Cd-Se DWFs.

\subsubsection{Cd-Se coordination numbers}

The Cd-Se coordination numbers for the core-shell nanorods are not easily explained. Certainly, the value for the cores is not surprising; it is similar to that measured for the longer CdSe nanorods discussed in chapter 3. Naïvely, however, assuming that the shell growth mechanism is correct, one would expect that as more $\mathrm{Cd}$ is added to a plain CdSe core, all of the dangling Se bonds would be capped. This would result in a coordination number of 4 for all core-shell nanorods. While this result is observed for the thin-shell nanorods, the medium- and thick-shell nanorods again show underbonded Se. It is not clear why the coordination number would decrease for thicker-shelled nanorods, unless additional interfacial strain could result in Cd vacancies. One additional possibility not yet accounted for is Se-S bonding; previous EXAFS studies showed that Se-Se bonding occurred in CdSe quantum dots [13]. 


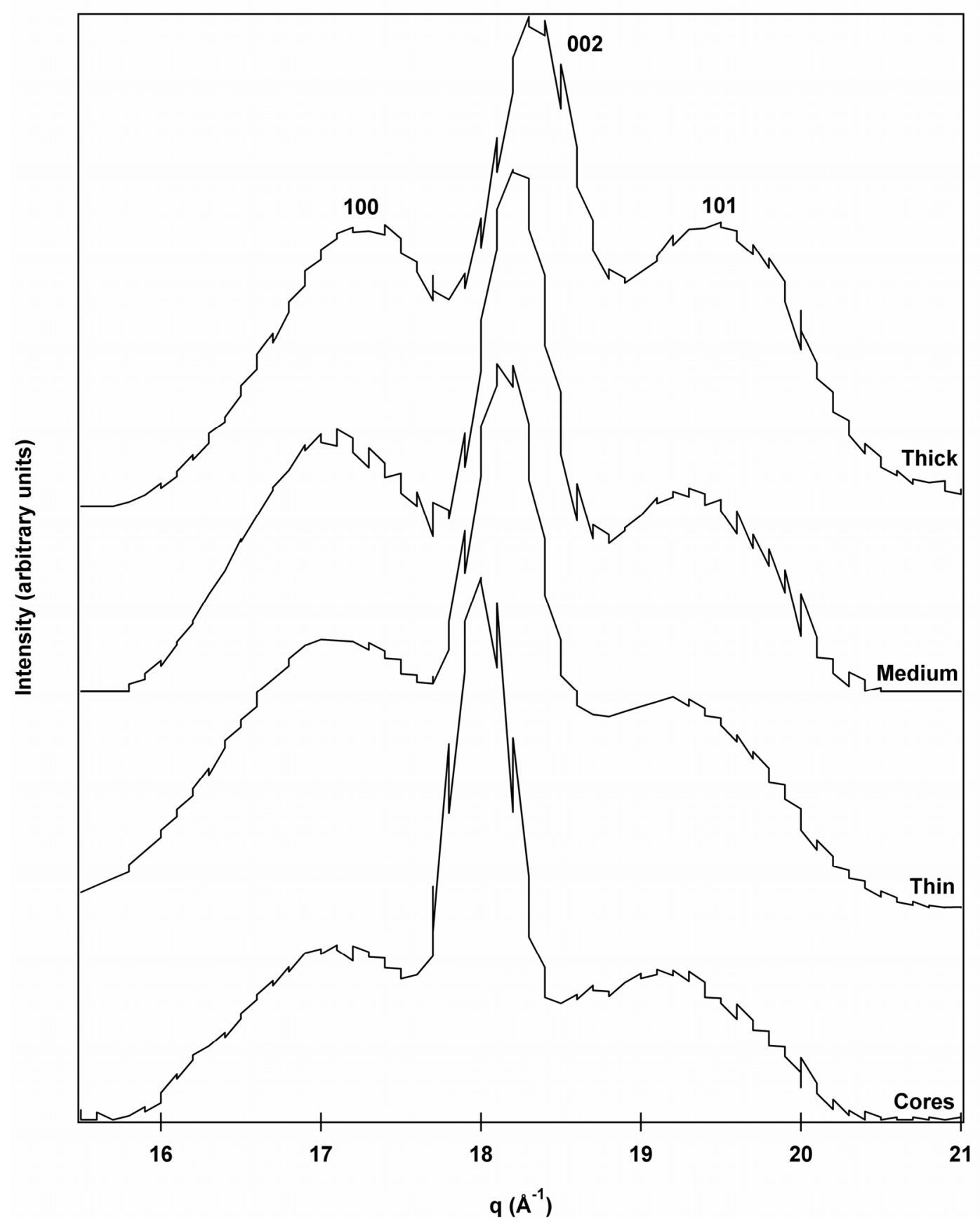

Figure 4.5. X-ray diffractograms from core and core-shell nanorods, featuring the first three strong reflections. As the shell thickness increases, these reflections shift to higher $q$ values, indicating a decrease in interplanar spacings. 


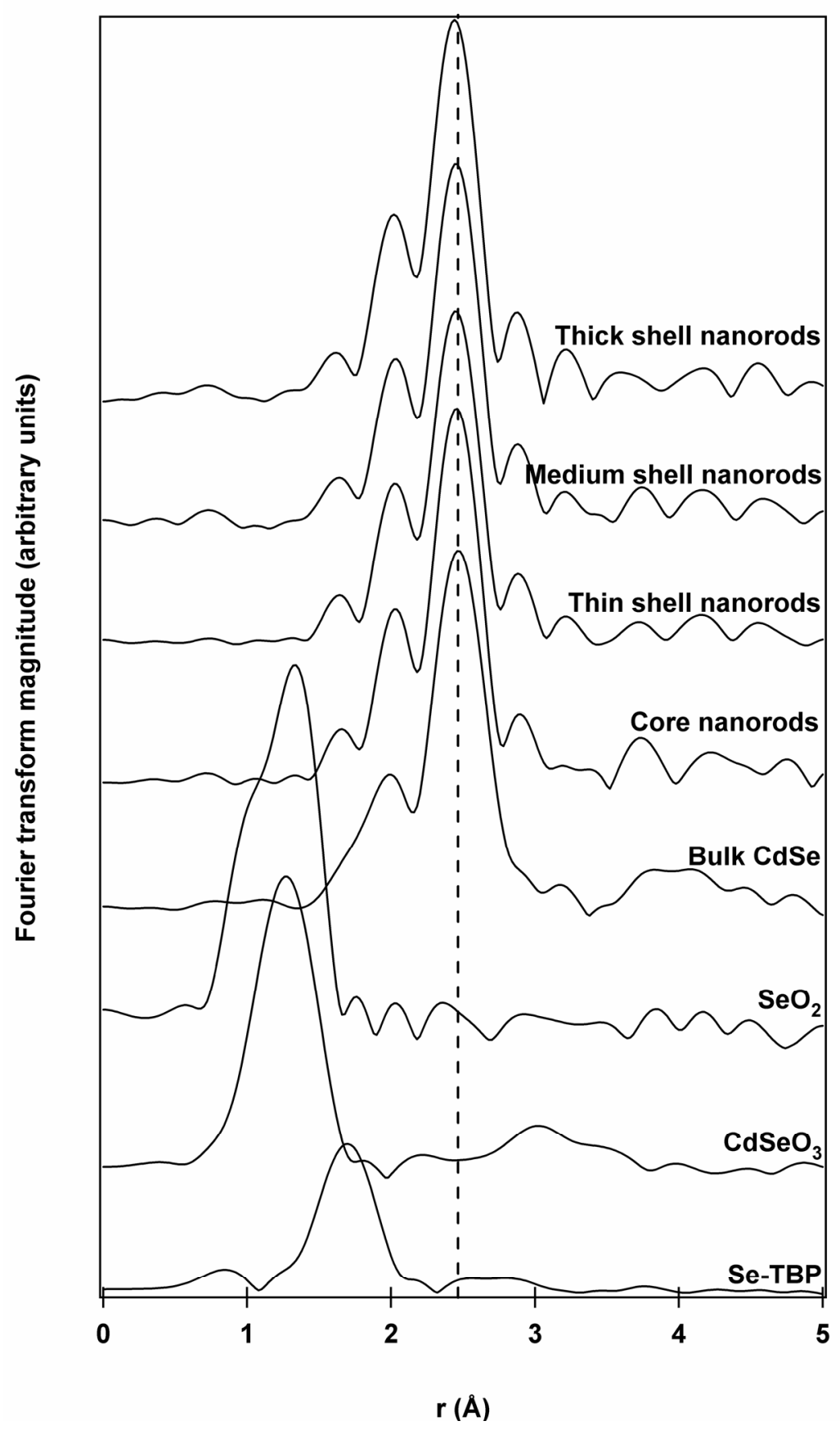

Figure 4.6. Fourier transform magnitudes from raw Se K-edge EXAFS data ( $k^{2}$-weighting).

Dotted line is centered on the first-shell peak for bulk CdSe. Se-TBP refers to Setributylphosphine, a synthesis precursor (see chapter 3); the oxide standards represent likely oxide surface species. 

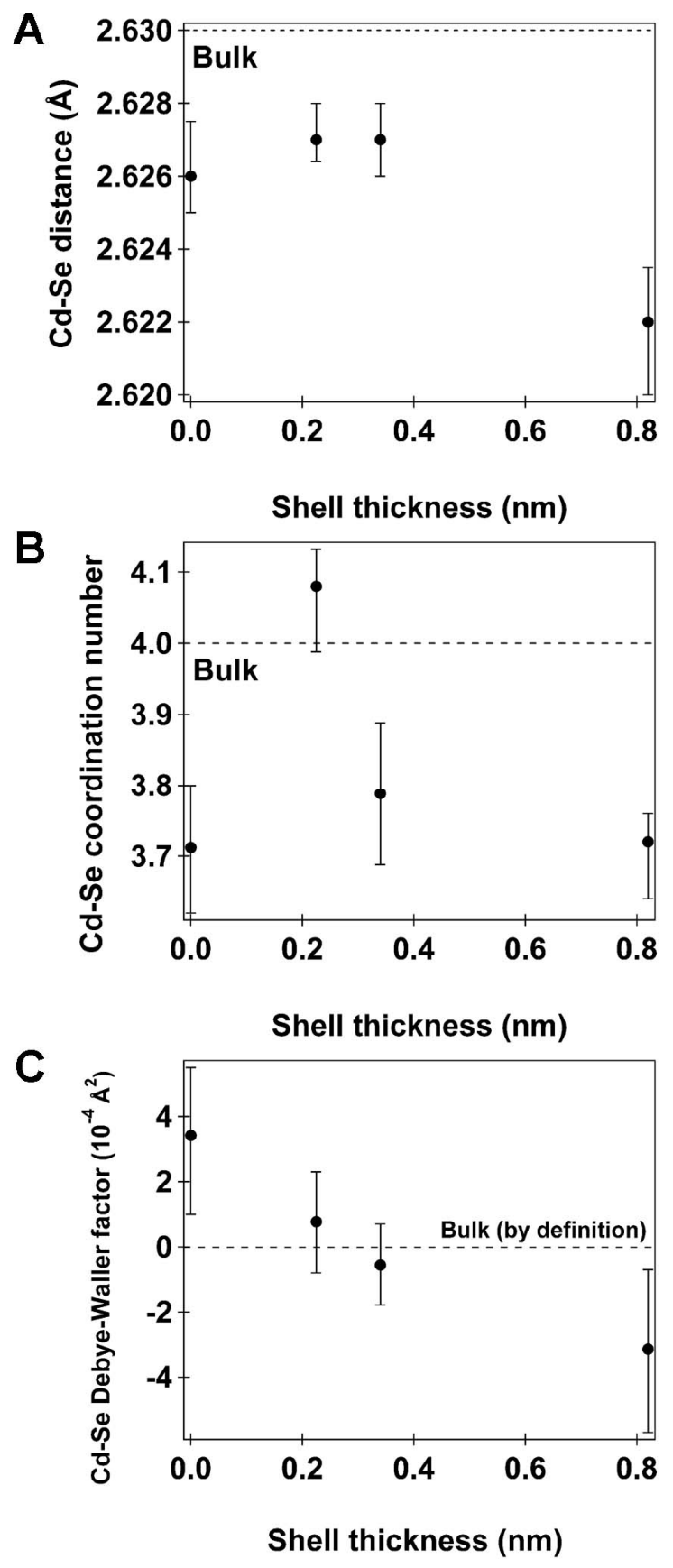

Figure 4.7. Structural parameters derived from Se K-edge EXAFS on core and core-shell nanorods. Error bars represent 1-sigma limits, and dashed lines denote bulk values. (A) Cd-Se distances. (B) Cd-Se coordination numbers. (C) Cd-Se Debye-Waller factors (relative to bulk). 


\subsubsection{Zn K-edge EXAFS}

Fourier transform magnitudes of raw $\mathrm{Zn}$ K-edge EXAFS are shown in figure 4.8. Principal component analysis was used to select standards for fitting. Final EXAFS fitting was done using phase and amplitude files derived from zinc sulfide and zinc phosphate $\left(\mathrm{Zn}_{3}\left(\mathrm{PO}_{4}\right)_{2}\right)$, a model compound for zinc-phosphonic acid surfactant complexes on the nanorod surfaces. Sphalerite $\mathrm{ZnS}$ data was used instead of wurtzite data as our bulk standard, as the first shells for both polymorphs are indistinguishable, and the sphalerite data was of higher quality.

\subsubsection{1. $\mathrm{Zn}-\mathrm{S}$ distances}

A plot of $\mathrm{Zn}-\mathrm{S}$ distances with respect to shell thickness is shown in figure $4.9 \mathrm{~A}$. What is most remarkable is the contraction of the $\mathrm{Zn}-\mathrm{S}$ distances despite being grown on top of two materials with lattice parameters larger than those of Zn-S. Normally, as has been demonstrated in other epitaxial systems, the $\mathrm{Zn}-\mathrm{S}$ lattice would be expected to expand in order to relieve some of the lattice mismatch strain.

It is possible that such bond contraction is a result of the $\mathrm{ZnS}$ being on the surface. For many crystalline surfaces, it is common for the first few surface monolayers to rearrange themselves to a new low-energy geometry, generally to maximize bonding interactions; such rearrangements may involve bond contractions [81]. The contraction lessens as the shell

thickens. This may be due to the fact that as the shell grows, a lower percentage of the total $\mathrm{ZnS}$ bonds are actually at the surface. The presence of a surface-induced contraction is consistent with the calculations discussed in chapter 3, although it is not clear why they would be so much more dramatic with a $\mathrm{ZnS}$ shell. 


\subsubsection{2. $\mathrm{Zn}-\mathrm{S}$ coordination numbers}

The Zn-S coordination numbers (figure 4.9 B) are not particularly surprising; as the shell thickens, the coordination number increases, much as would be expected. EXAFS detects the presence of zinc bonding to a surfactant species (the fitting error decreases when zinc phosphate is included as opposed to zinc oxide). Currently, the average number of surfactant molecules per zinc atom cannot be quantified, due to the nature of the empirical standard used for fitting, zinc phosphate, which has an ill-defined first shell (multiple $\mathrm{Zn}-\mathrm{O}$ distances). A more quantitative analysis necessitates the use of FEFF to directly simulate EXAFS from surfactant species.

\subsubsection{Zn-S Debye-Waller factors}

Despite the fact that $\mathrm{ZnS}$ is on the surface, the DWFs (figure $4.9 \mathrm{C}$ ) do not vary greatly from bulk (defined as zero). Normally, due to underbonding, surfaces, particularly of nanocrystals, are expected to be disordered with respect to the bulk. It is possible that the static disorder that would be expected for a surface is balanced out by decreased vibrational freedom due to surface-induced compression. 


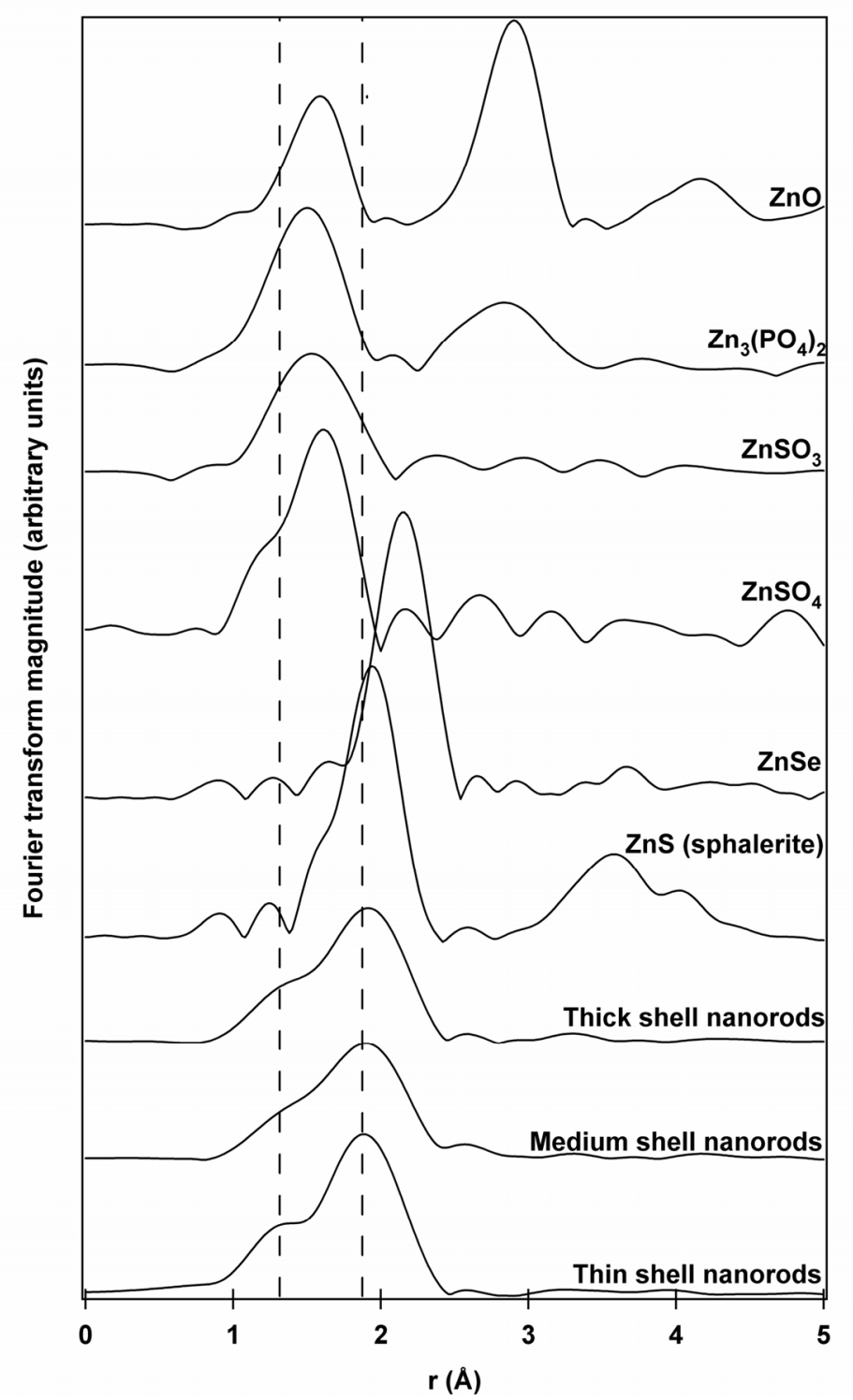

Figure 4.8. Fourier-transform magnitudes of Zn K-edge raw data. Dashed lines serve to guide the eye with respect to the peaks shown in the thin shell nanorod data. Sphalerite $\mathrm{ZnS}$ data is shown, as it is virtually identical to that for wurtzite and the data was of higher quality. 

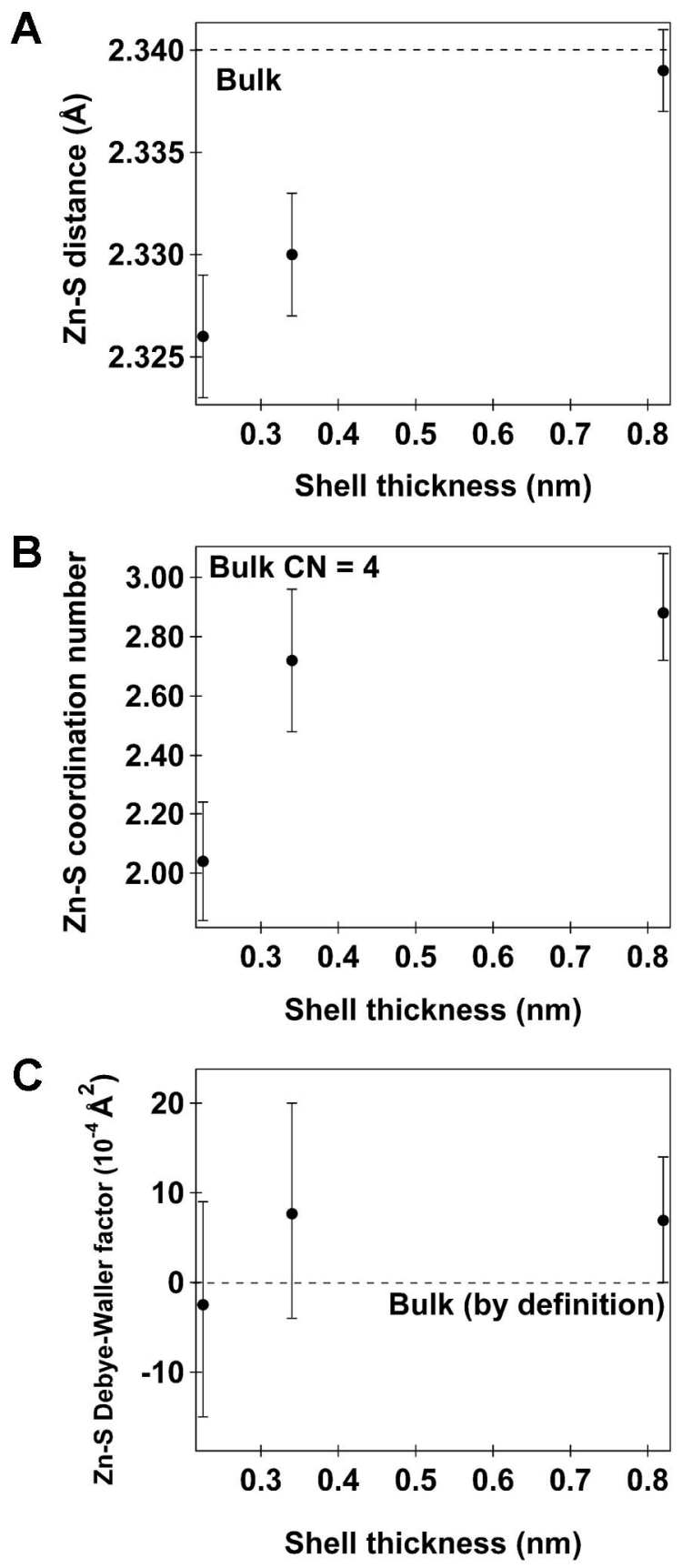

Figure 4.9. Physical parameters for first-shell Zn-S bonds derived from Zn K-edge EXAFS. Error bars represent 1-sigma limits, and dashed lines denote bulk values. (A) Zn-S distances. (B) ZnS coordination numbers. (C) Zn-S Debye-Waller factors (relative to bulk). 


\subsection{3. $\mathrm{Cd} \mathrm{L}_{3}$-edge EXAFS}

The Cd EXAFS signal-to-noise ratio was much poorer than that of the Se and Zn, primarily because of the lower absorption cross section, as well as the reduced flux for this energy range. Principal component analysis was performed upon the Cd XANES data to select for the major chemical species contributing to the $\mathrm{Cd}$ signal. The two main species were CdS and CdSe. The Cd EXAFS was fit with both CdS and CdSe shells. Due to the lower signal-to-noise ratio, the k-range of this data was shortened. In order to keep the number of parameters fit below the number of the degrees of freedom in the data, values of distance and the Debye-Waller factor were fixed for the Cd-Se shell to those derived from Se EXAFS.

The Fourier transform magnitudes of the raw Cd EXAFS data are displayed in figure 4.10. In figure 4.11, plots of the Cd-S distances, the coordination numbers, and the DebyeWaller factors with respect to shell thickness are shown. The Cd-S distances do not differ from bulk, even though the shell has been grown onto CdSe, which has a larger lattice spacing. It is possible that the $\mathrm{ZnS}$ shell causes a compressive strain which cancels out any effect of the underlying CdSe lattice.

The Cd-S coordination number shows the same trend observed for the Cd-Se coordination number; the thinnest shell has the highest coordination number, followed by a reduction for the thicker shells. Again, this may imply that upon continued growth of $\mathrm{ZnS}$, vacancy formation is induced at the interface. An alternative possibility is that of a vacancyridden mixed ZnS/CdS shell (previous energy-dispersive X-ray analyses have indicated that the shells contain small amounts of $\mathrm{Cd}$ beyond the interface [72]). The presence of Se-S 
bonding might also explain such anomalous coordination numbers [13]. The DWFs for the Cd-S are either positive or zero, indicating the presence of some disorder. 


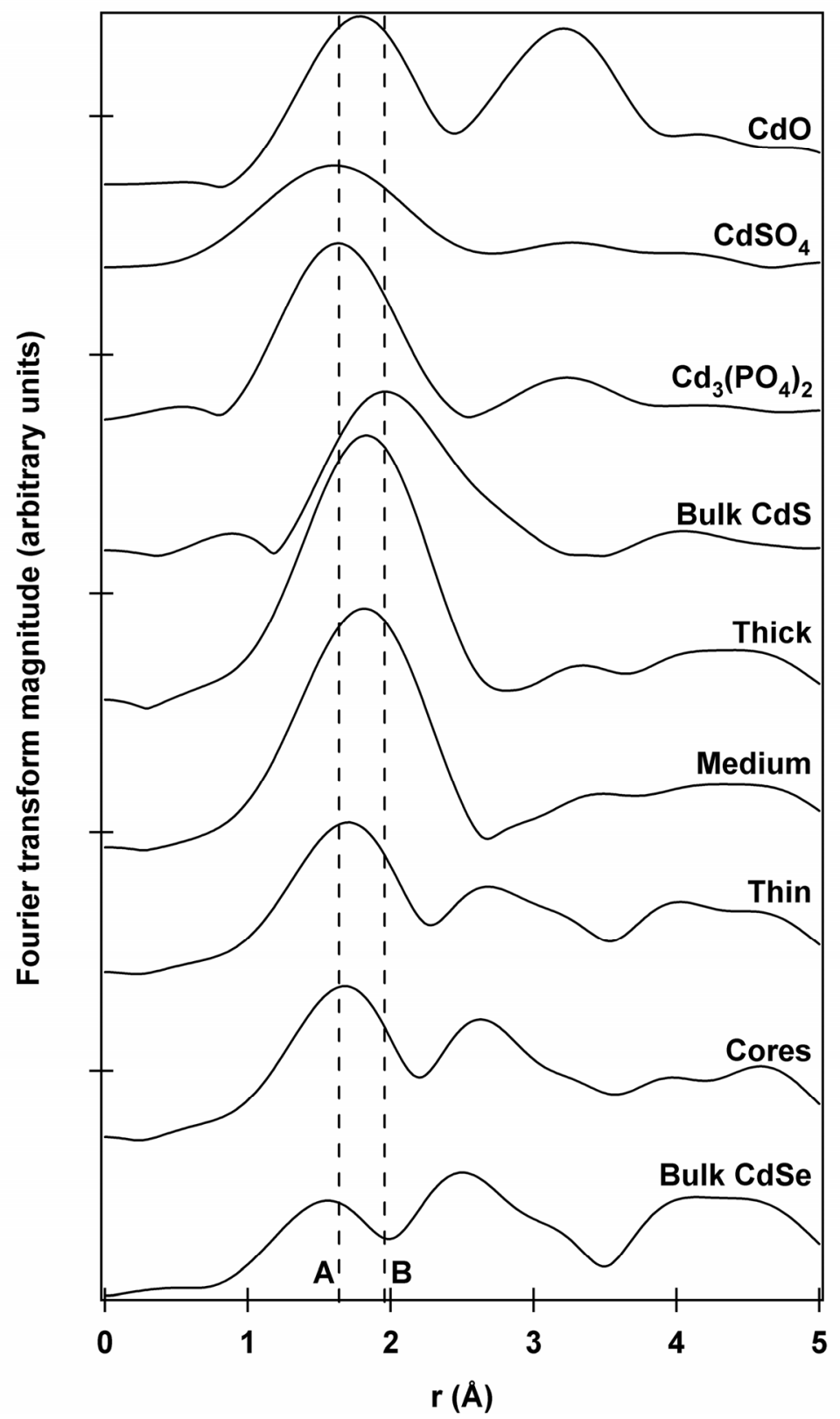

Figure 4.10. Fourier-transform magnitudes of $\mathrm{Cd} \mathrm{L}_{3}$-edge raw data. Dashed line $\mathrm{A}$ is centered on the $\mathrm{Cd}_{3}\left(\mathrm{PO}_{4}\right)_{2}$ peak, while line $\mathrm{B}$ is centered on the bulk $\mathrm{CdS}$ first shell peak. Bulk CdSe and the nanorod signals are multiplied by 3 for easier visual comparison. 

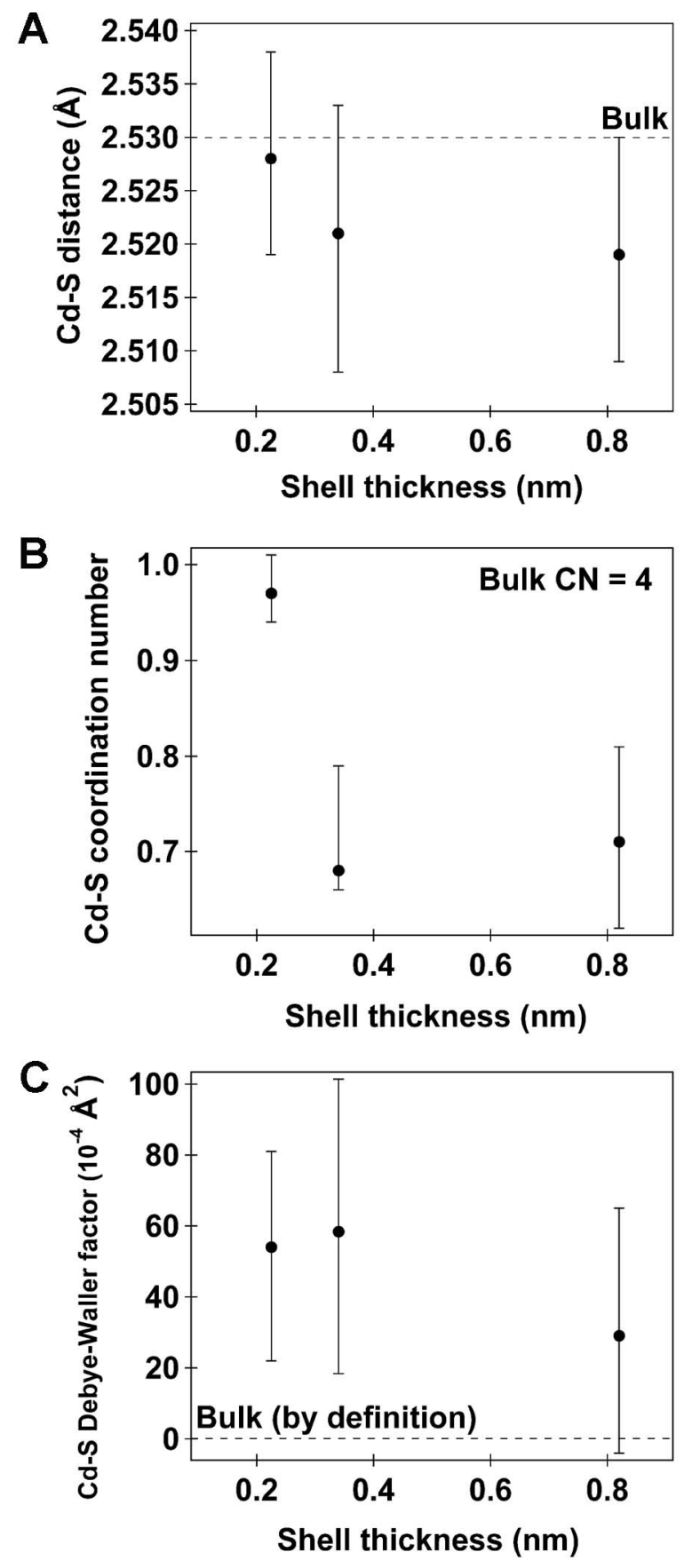

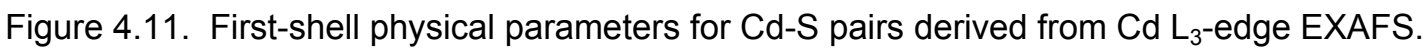

Error bars represent 1-sigma limits, and dotted lines denote bulk values. (A) Cd-S distances. (B) Cd-S coordination numbers. (C) Cd-S Debye-Waller factors (relative to bulk). 


\subsubsection{S K-edge XAS}

S K-edge XAS was collected from multiple nanorod samples, as well as standards. Unfortunately, despite the helium-filled beam path, the signal-to-noise ratio was still poor. Analyses of EXAFS has produced inconsistent results. Principal-component analysis and linear-combination fitting of XANES has also produced results incongruent with other spectra (e.g. showing that no $\mathrm{ZnS}$ is present when the $\mathrm{Zn}$ EXAFS has displayed a strong signal). Further analyses are underway to see if such incongruities in the fitting might be resolved.

\subsection{Outlook and conclusions with respect to previous work on nanoscale epitaxial structures}

Despite the great variety of core-shell colloidal semiconductor nanocrystal syntheses described in the literature, there is still an astonishing paucity of detailed structural studies concerning these materials. One EXAFS study concerning CdTe quantum dots [82] capped with thiols displayed trends both contrary to and in agreement with our observations. This study found that relative to bulk, Cd-S bonds were elongated, while some Cd-Te bonds (attributed to the surface) were constricted. In the core-shell nanorods, the $\mathrm{Zn}-\mathrm{S}$ bonds were constricted, and the core constriction, while present, was not nearly as dramatic. The study on the quantum dots also showed that the Debye temperature decreased for the CdTe core with respect to bulk, indicating increased stiffness (decreased temperature-dependent disorder), while the static disorder increased with respect to bulk. Debye-Waller factors for the core in core-shell nanorods decreased with respect to the bulk. Note that these DWFs 
are a measurement of overall disorder, inclusive of both temperature-dependent and static effects.

More regarding II-VI heteroepitaxial structures or mixed II-VI semiconductor structures may be found in bulk or thin-film literature, although there exists further room for investigations (especially EXAFS investigations sensitive to local structure or vacancies). It should be noted that the structures of these bulk or thin films may heavily depend upon their preparation conditions; thus, any comparisons between these structures and those of the nanocrystals must be considered carefully. For the most part, only broadly general comparisons can be made.

The observations of bond relaxations for mixed semiconductors and thin-film superlattices have varied in the literature. Generally, they either show bond relaxations, in which smaller lattices stretch to accommodate larger ones and vice versa [83-85]; at times, there are minimal to no changes in bond distances with respect to the bulk [86].

Nevertheless, despite the variability in the literature, it appears that the bond distance contraction observed for the $\mathrm{ZnS}$ nanorod shell cannot be explained according to what has been observed for bulk/thin-film systems. We have not been able to find any examples in which a material epitaxially grown onto or mixed with another material with a larger lattice parameter contracts. This indicates that such contractions must be surface-induced or otherwise the result of the unique $1 \mathrm{D}$ geometry of the system, in between a quantum dot and a thin film. A Raman study of core-shell CdSe-ZnS quantum dots indicated that thinner $\mathrm{ZnS}$ shells were amorphous and only grew more crystalline with increasing thickness [87]. If a similar phenomenon is occurring in the nanorods, this might explain anomalous bond distances. Regarding the possibility of either switched anions/cations or defects at the 
epitaxial interface, the bulk/thin-film literature contains examples of both [84, 88-90], indicating that both are possible for the nanorods. The results of the EXAFS studies in this chapter have ruled out the former phenomenon, but the possibility of vacancy defects [91] must be taken into account more quantitatively for future analyses.

Further analysis of the core-shell nanorod data is necessary for a more complete picture of their structures. First, as mentioned in section 4.4, the analysis of the sulfur XAS will need to be completed. If S-Se bonding appears to be present, XPS may be a useful complementary technique for independent detection of Se-S bonds. Second, simulations of the XRD patterns for nanorods could be very useful to see if simultaneous bond expansions and contractions would be consistent with the observed XRD. Such simulations might resolve the apparent discrepancies between the XRD and EXAFS data. 


\section{CHAPTER 5. INTRODUCTION TO ELECTRON DIFFRACTION}

\subsection{Background and motivation}

Diffraction is an immensely useful tool for the structural characterization of materials. This technique is an integral part of some of the studies in this dissertation, discussed in chapter 6 . Therefore, a brief introduction to the principles of diffraction is presented in this chapter. Diffraction is defined as the bending or scattering of traveling waves by an obstruction [32]. The intensity of the resulting waves, called a diffraction pattern, depends upon the structure of the obstacle. Diffraction patterns are particularly helpful for characterizing periodic arrangements of obstacles, such as the periodic array of atoms in a crystal.

Electron diffraction was specifically used for the studies described in chapter 6 . Thus, the discussion in this chapter focuses in particular on electron diffraction and how it is coupled with electron microscopy to characterize structure [92][93-96]. As the majority of readers are the most familiar with $\mathrm{X}$-ray diffraction (XRD), attempts are made to draw comparisons between XRD and ED.

\subsubsection{The Bragg description of diffraction}

W.L. Bragg provided an early description of X-ray scattering from crystalline materials [93]. He envisioned a crystal as being composed of parallel planes of atoms separated by a distance $d$. When X-rays strike these planes, they are specularly reflected. 
Consequent constructive or destructive interference between the rays gives rise to periodic scattering patterns.

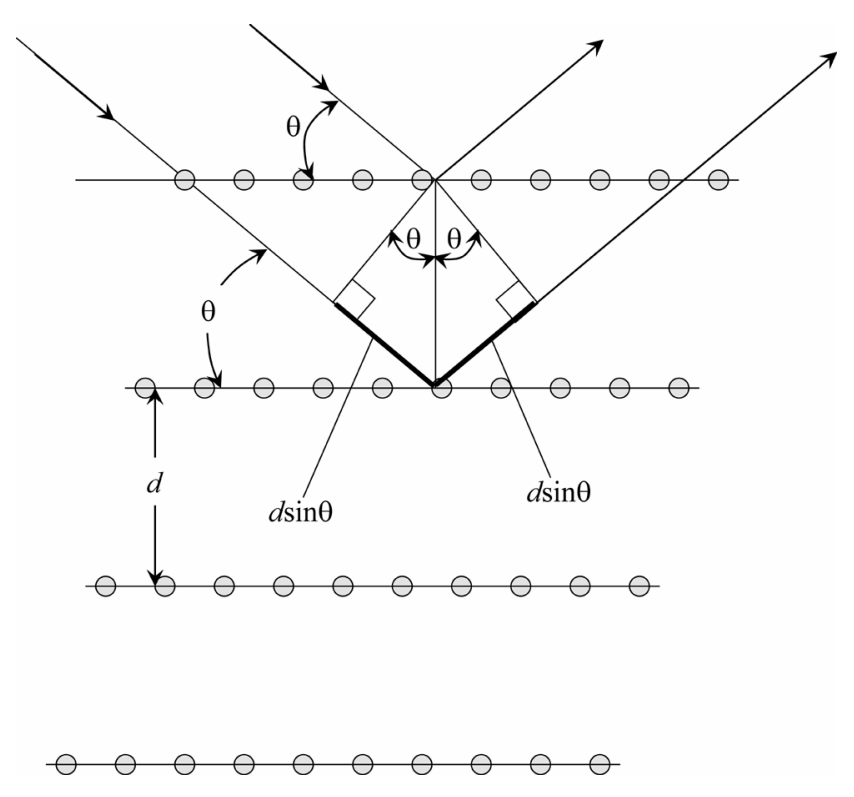

Figure 5.1. Schematic of Bragg diffraction model.

A schematic of X-ray scattering from a set of planes is depicted in figure 5.1. In order for the two X-rays depicted to interfere constructively, the difference in path length between them equaling $2 d \sin \theta$ (where $\theta$ is the angle of incidence) must equal an integral number of wavelengths. This requirement leads to the Bragg equation,

$$
n \lambda=2 d \sin \theta
$$

where $n$ must be an integer, and $\lambda$ is the wavelength of the incident radiation. This description illustrates the link between the positions of intense peaks in the diffraction pattern and spacings of crystallographic planes. Furthermore, given a single crystalline 
sample and a monochromatic source, $\theta$ is constrained to a number of values. Thus, it is also (correctly) predicted that a very restricted set of orientations (characterized by $\theta$ ) give rise to observation of one or few pairs of diffraction spots. Indeed, for simple analyses of diffraction data, the Bragg equation is still heavily used (see figure 5.2 for a schematic of an X-ray diffraction experiment).

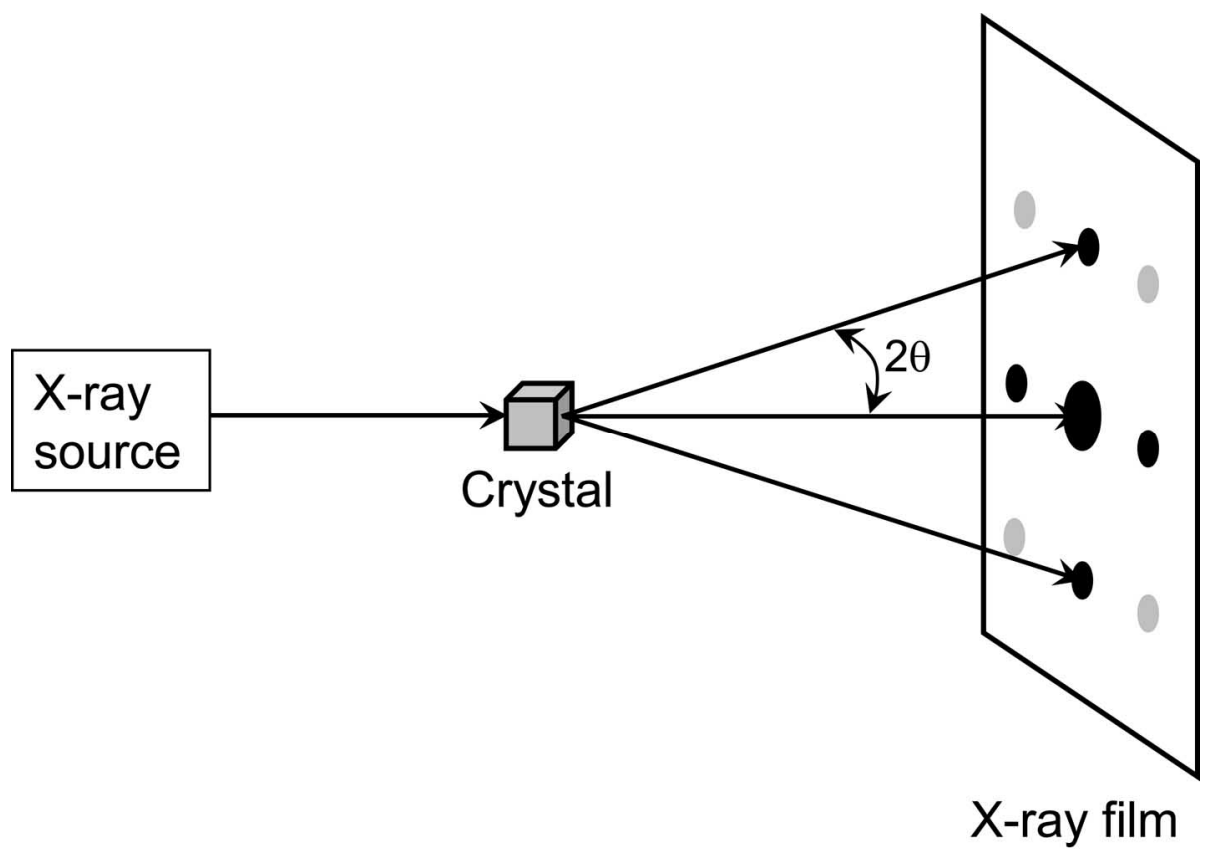

Figure 5.2. Schematic of a typical diffraction experiment. The experimenter already knows the wavelength of the incident radiation. By knowing the geometry of the setup, she can measure distances between reflections on the film, derive the angle of diffraction, and use Bragg's Law to quickly obtain $d$-spacings. 


\subsubsection{Real and reciprocal space}

A crystal consists of a periodic array, generally of atoms (although nanocrystals can form their own periodic arrays, which will be discussed in chapter 6). The Bravais lattice, defined as the periodic set of points situated in indistinguishable environments, describes the underlying periodicity of the lattice, rather than the locations of specific atoms [93]. (The geometries of specific atoms will be addressed in section 5.1.5.) A three-dimensional Bravais lattice can be described as the points with position vectors

$$
\vec{R}=n_{1} \vec{a}+n_{2} \vec{b}+n_{3} \vec{c} .
$$

The position vectors $\vec{a}, \vec{b}$, and $\vec{c}$ are the translations of the unit cell (the smallest repeatable unit in the structure), and $n_{1}, n_{2}$, and $n_{3}$ are integers.

Now we have a way to describe the crystal structure, but this is not sufficient for a discussion of diffraction. Note that DPs are caused by the presence of periodic structural motifs; in fact, when using Bragg's Law to interpret a scattering pattern, we derive the periodic lattice spacings rather than any real-space vectors. Intuitively, it behooves us to express diffraction phenomena directly in terms of these spacings.

The problem is much simplified in 'reciprocal space', in which each vector $\vec{R}^{*}$ is associated with a particular set of planes in a crystal, hence a particular periodicity. In other words, reciprocal space and real space are related by a Fourier transform. The reciprocal space vectors $\vec{R}^{*}$ satisfy the relation [93]

$$
e^{i \vec{R}^{*} \cdot \vec{R}}=1 .
$$

For a given reciprocal space vector $\vec{R}^{*}=h \vec{a}^{*}+k \vec{b}^{*}+l \vec{c}^{*}$, 


$$
\vec{a}^{*}=\frac{\vec{b} \times \vec{c}}{\vec{a} \cdot(\vec{b} \times \vec{c})}, \vec{b}^{*}=\frac{\vec{c} \times \vec{a}}{\vec{a} \cdot(\vec{b} \times \vec{c})}, \text { and } \vec{c}^{*}=\frac{\vec{a} \times \vec{b}}{\vec{a} \cdot(\vec{b} \times \vec{c})}
$$

$\vec{R}^{*}$, commonly denoted in the electron diffraction literature as $\vec{g}_{h k l}$, has the following important properties relative to real space [94]:

(1) $\vec{g}_{h k l}$ is normal to the real-space plane that cuts the $a, b$, and $c$ axes of the unit cell at $1 / h$, $1 / k$ and $1 / l$, respectively. Indices $h, k$, and $l$ are are commonly known as the Miller indices of this plane. The set of planes to which $\vec{g}_{h k l}$ is normal is referred to as the (bkl) planes.

(2) $d_{h k l}=\frac{2 \pi}{\left|\vec{g}_{h k l}\right|}$, that is, the length of $\vec{g}_{h k l}$ is inversely proportional to the spacings between the real-space planes $(b k)$.

\subsubsection{The Laue description of diffraction [97]}

Consider two identical sets of atoms placed at the positions $\vec{R}$ of a Bravais lattice. These atoms re-radiate incident radiation isotropically as spherical waves; the outgoing waves interfere constructively only along certain directions for a monochromatic source.

The two sets of atoms are separated by a displacement vector $\vec{d}$, as illustrated in figure 5.3 . (Note that $\hat{n}_{I}, \hat{n}_{F}$, and $\theta$ are defined in figure 5.3). 


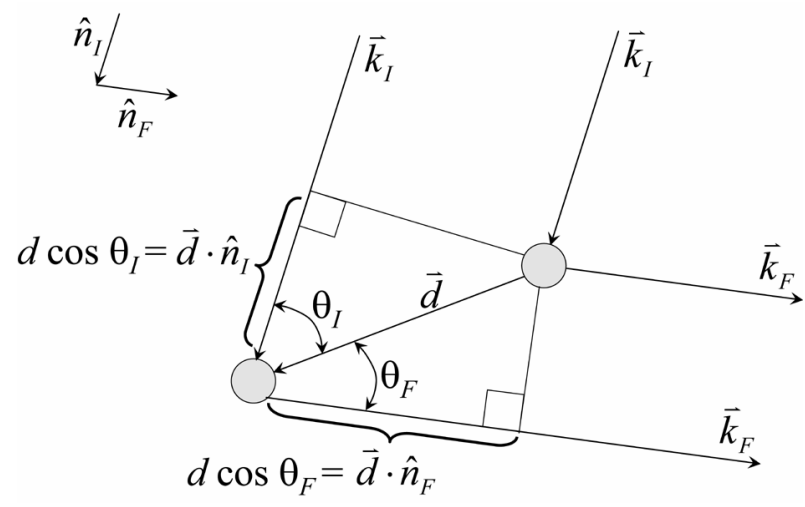

Figure 5.3. Schematic for discussion of Laue description of diffraction, showing the path difference when radiation or particles are scattered from two points separated by $\vec{d}$. The vectors $\vec{n}_{I}, \vec{n}_{F}$ correspond respectively to the direction of the incident beam and the direction of the vector connecting the sample and the detector measuring diffracted intensity. Image adapted from N. W. Ashcroft, and N. D. Merman, Solid State Physics (Brooks Cole, Pacific Grove, CA, 1976).

These atoms are struck by incident X-rays (or electrons, or neutrons) traveling in the direction $\hat{n}_{I}$ with a wavelength $\lambda$. The incident waves can be described according to their momentum wavevector $\vec{k}_{I}=2 \pi \hat{n}_{I} / \lambda$. In order for a maximum in the intensity of scattered rays to be seen for the momentum wavevector $\vec{k}_{F}=2 \pi \hat{n}_{F} / \lambda$, the path difference between the rays scattered towards this direction must be equal to an integral number of wavelengths for constructive interference. From figure 5.3 we can see that this relationship can be expressed simply as

$$
d \cos \theta_{I}+d \cos \theta_{F}=\vec{d} \cdot\left(\hat{n}_{I}-\hat{n}_{F}\right)=m \lambda
$$

Multiplying both sides of this equation by $2 \pi / \lambda$, we obtain

$$
\vec{d} \cdot\left(\vec{k}_{I}-\vec{k}_{F}\right)=2 \pi m .
$$


For an entire lattice of points, in which the points are separated from each other by the Bravais lattice vectors $\vec{R}$,

$$
\vec{R} \cdot\left(\vec{k}_{I}-\vec{k}_{F}\right)=2 \pi m
$$

which alternatively can be expressed as

$$
e^{i \vec{R} \cdot\left(\vec{k}_{I}-\vec{k}_{F}\right)}=1
$$

The Laue condition for diffraction is realized upon combining this expression with equation 5.3, in which the reciprocal lattice was defined. Constructive interference occurs only when

$$
\vec{k}_{I}-\vec{k}_{F}=\vec{g}_{h k l}
$$

that is, when the change in the momentum wavevector is also a vector of the reciprocal lattice [94].

The ability of the Laue description to retrieve the empirically correct Bragg equation is illustrated in figure 5.4. The magnitude of the vector $\vec{k}_{I}-\vec{k}_{F}$ is $4 \pi \sin \theta / \lambda$. We also know that when $\vec{k}_{I}-\vec{k}_{F}$ fulfills the Laue conditions for diffraction it is equal to $\vec{g}_{h k l}$, and that $d_{h k l}=\frac{2 \pi}{\left|\vec{g}_{h k l}\right|}$. Thus, we obtain the equation $4 \pi \sin \theta / \lambda=2 \pi / d_{h k l}$, which reduces to equation 5.1 .

\subsubsection{The Ewald sphere}

A convenient geometric construction, known as the Ewald sphere, aids visualization of the Laue diffraction conditions and is depicted in figure $5.4[93,94]$. In reciprocal space, the incident X-ray (or electron, or neutron) wavevector $\vec{k}_{I}$ is drawn such that its beginning is at the center of a sphere; the vector ends on the origin O. The Ewald sphere has a radius of 
$\left|\vec{k}_{I}\right|\left(\right.$ also $\left.\left|\vec{k}_{F}\right|\right)$. The wavevector for the scattered X-ray $\vec{k}_{F}$ is drawn with its beginning at the center as well. Using basic vector addition, the difference $\vec{k}_{I}-\vec{k}_{F}$ is thus depicted. Per equation 5.8 , constructive interference can occur only when $\vec{k}_{I}-\vec{k}_{F}$ equals a reciprocal space vector $\vec{g}_{h k l}$. In other words, if the sphere intersects a given point $(h, k, l)$ in reciprocal space, there should be constructive interference from the ( $h k l)$ set of real space planes.

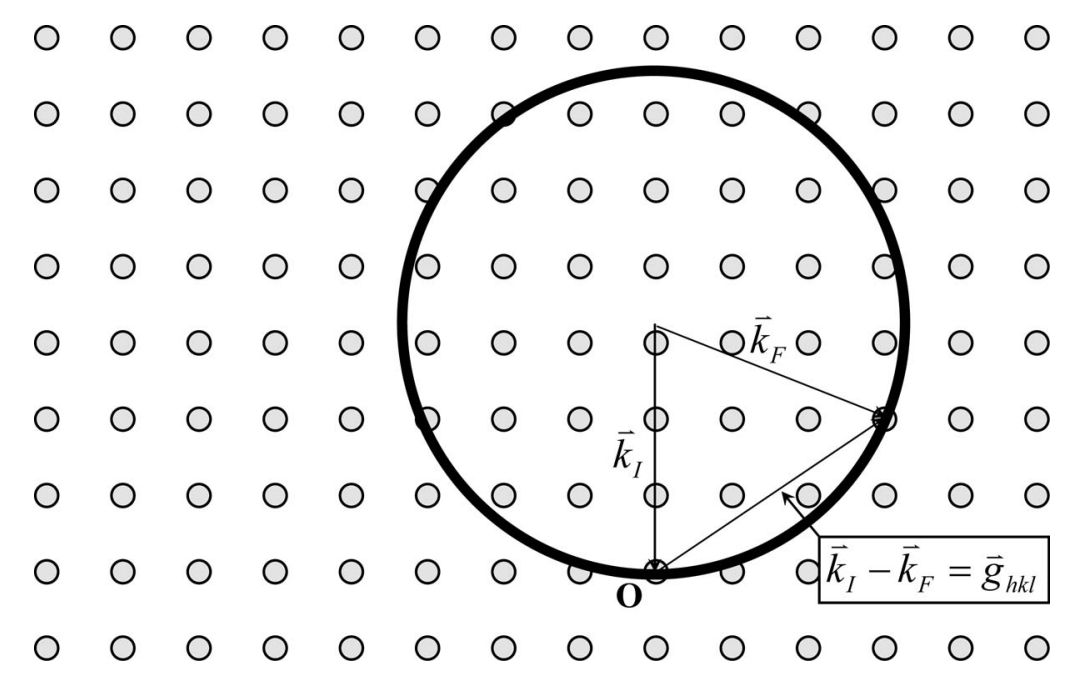

Figure 5.4. Schematic of a typical 2D cross-section of an Ewald sphere for X-rays. $\vec{k}_{I}$ is the wavevector of the X-ray incident upon the crystal, $\vec{k}_{F}$ is the wavevector of the diffracted X-ray. The vector $\vec{g}_{h k l}$ is a vector of the reciprocal lattice.

\subsubsection{The structure factor [98]}

So far in this review of diffraction, we have not included the effect of the geometry and composition of atoms in a given unit cell of the crystal. When collecting diffraction patterns from real crystals, not every set of planes ends up producing a diffraction signal. 
Consider a real-space unit cell with identical atoms at positions $\vec{d}_{1}$ and $\vec{d}_{2}$. Incident X-rays (both with the same wavevector) scatter from these atoms; the change in wavevector is $\vec{g}_{h k l}$ (satisfying Laue conditions for diffraction). The phase difference between the X-rays is $\vec{g}_{h k l} \cdot\left(\vec{d}_{1}-\vec{d}_{2}\right)$ (per figure 5.3 ), and the amplitudes of these rays will differ by a factor of $e^{i \vec{g}_{h k l} \cdot\left(\vec{d}_{1}-\vec{d}_{2}\right)}$. The amplitudes of the rays scattered are in the ratios $e^{i \vec{g}_{h k l} \cdot\left(\vec{d}_{1}\right)}$ and $e^{i \vec{g}_{h k l} \cdot\left(\bar{d}_{2}\right)}$. Summing up the contributions of these two atoms in the unit cell to the scattered X-ray produced by the entire unit cell, we have an amplitude with the factor

$$
S_{K}=\sum_{j=1}^{n} e^{i \vec{g}_{h k l} \cdot \vec{d}_{j}}
$$

This is known as the geometrical structure factor, and the intensity of any diffracted beam is proportional to $\left|S_{K}\right|^{2}$. For systems with non-identical atoms, this structure factor has the form

$$
S_{K}=\sum_{j=1}^{n} f_{j}\left(\vec{g}_{h k l}\right) e^{i \vec{g}_{h k l} \cdot \vec{d}_{j}},
$$

where $f_{j}\left(\vec{g}_{h k l}\right)$ is the atomic form factor, a function specific to the particular scattering atom $j$.

The structure factor explains the variations of intensities among the diffraction peaks corresponding to different reciprocal space vectors. Particularly important is the prediction of so-called "forbidden" reflections. These refer to complete absence of diffracted intensity in conditions where Bragg's equation would predict observation of non-zero intensity. These turn out to be cases in which $S_{\mathrm{K}}=0$, usually due to a real-space symmetry for the crystal structure under consideration. While we can rely on the structure factor to predict 
which reflections are forbidden for systems in which kinematical scattering (single-event, elastic) of waves can be assumed, this does not take into account dynamical scattering (multiple scattering) phenomena. Dynamical effects are non-negligible for electron diffraction, complicating the interpretation of diffraction data [94-96].

\subsection{Electron diffraction (ED)}

The previous material universally applies to diffraction with any form of radiation, including electrons. Nevertheless, ED has its own idiosyncrasies. In this section, the special features of ED are contrasted with the already discussed features of XRD.

\subsubsection{Energy range of electrons as opposed to X-rays}

Electron diffraction data is often collected in a transmission electron microscope (TEM); this was done for the studies discussed in chapter 6. In a TEM, electrons are commonly accelerated through voltages from 100-300 kV. The corresponding de Broglie wavelength is on the order of picometers, two orders of magnitude smaller than the value for X-rays. Given the smaller wavelength of electrons, the corresponding Ewald sphere is much larger, as depicted in figure 5.5. One consequence of a large Ewald sphere is that it contacts many points in reciprocal space-for a single orientation of the crystal, multiple sets of lattice planes may simultaneously give rise to diffraction spots. 


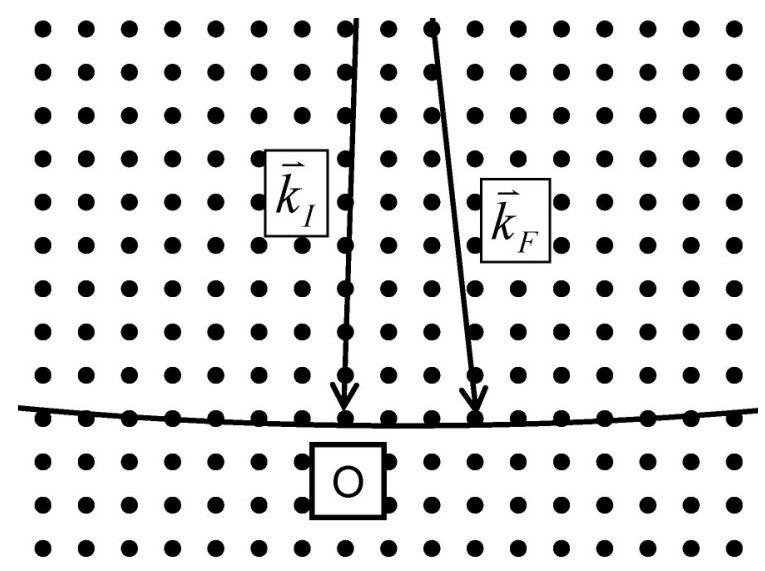

Figure 5.5. Schematic cross section of Ewald sphere for high-energy electrons. (Image not exactly to scale.)

\subsubsection{Additional idiosyncrasies of electron diffraction}

\subsubsection{Relrods}

As previously mentioned, reciprocal space is a Fourier transformation of real space. Each point in reciprocal space represents an infinite set of parallel planes of a given spacing. However, real TEM samples are not infinitely large; they are thinned down to dimensions $<100 \mathrm{~nm}$ for electron transparency, or may contain crystal domains $<100 \mathrm{~nm}$. When a crystalline solid is not infinite in extent, the resulting Fourier transform is no longer a set of discrete points, each corresponding to one spatial frequency; rather, these points are smeared out. These diffuse points are referred to as relrods (figure 5.6). The relrods depicted in figure 5.6 are from a thin film. They are elongated along the thin direction of the film (normal to the plane of the film). For a material that is $<100 \mathrm{~nm}$ in multiple directions, such 
as a spherical nanocrystal, the distributions in reciprocal space would be spherically symmetric.

Relrods are particularly important because they "relax" diffraction conditions. In figure 5.6, the black arrows point to some of the relrods touching the Ewald sphere. If these relrods were contracted down to points, they would not touch the sphere, and hence these sets of planes would not diffract. The presence of relrods can make electron diffraction patterns harder to interpret than XRD patterns.
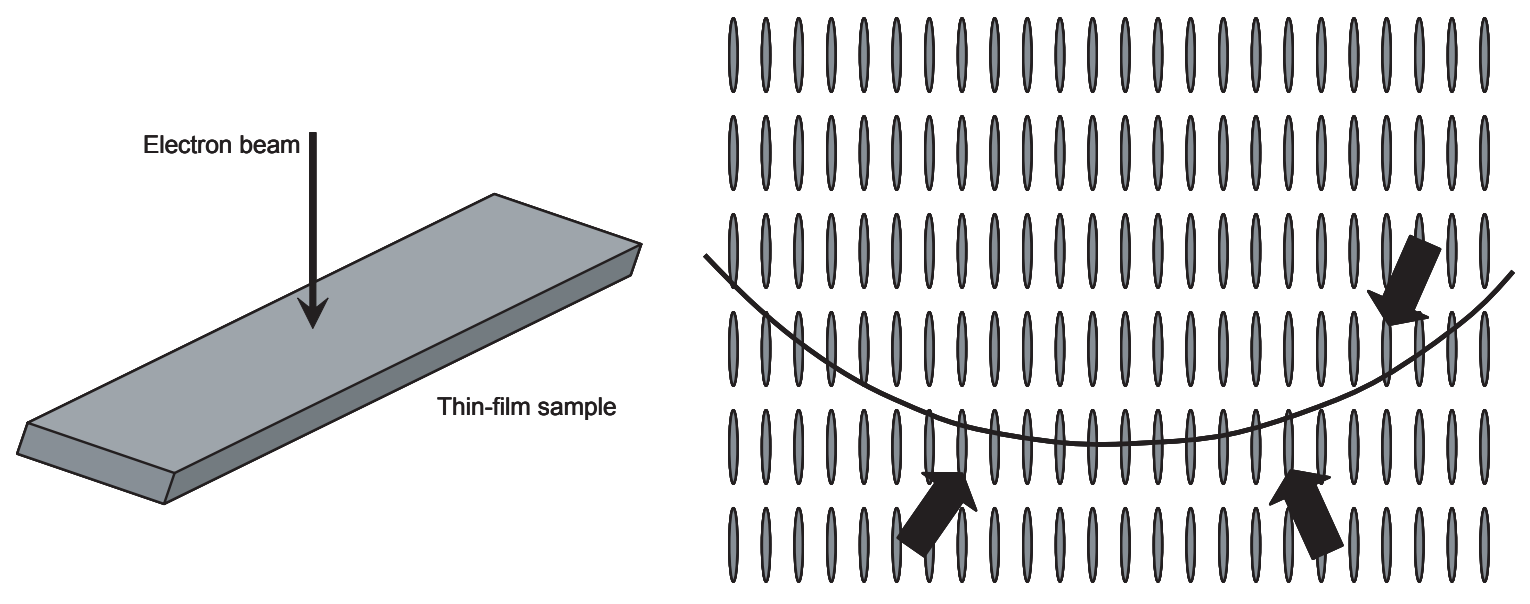

Figure 5.6. Materials with dimensions $<100 \mathrm{~nm}$ can result in rods (or other frequency distributions) rather than discrete points in reciprocal space. An example of relrods for a thin film sample is depicted. The black arrows point to relrods in reciprocal space touching the Ewald sphere. For a thick, single crystalline sample, these rods would be points and would not be touching the sphere, and hence we would not see a diffraction signal from these sets of crystal planes.

\subsubsection{Dynamical scattering of electrons}

Generally, any basic discussion of diffraction assumes kinematic scattering in which waves are scattered only once. Scattering events are assumed to be elastic. On the other 
hand, electrons interact strongly with atoms, leading to inelastic scattering. Scattered electron waves also interact with each other. Dynamical scattering, in which electrons are subjected to multiple scattering events, can make electron diffraction more difficult to interpret than XRD. First, the interaction of scattered electrons can lead to phenomena such as double diffraction, in which normally structurally forbidden reflections appear in the diffraction pattern. Second, dynamical scattering makes it difficult to obtain quantitative information from the intensities of different reflections.

\subsection{Electron microscopy}

Often, electron diffraction of materials is performed in an electron microscope. Electron diffraction patterns and microscopic images from the same sample contain complementary information. In addition to doing electron diffraction and imaging, many electron microscopes also allow for electron spectrometry, which can provide chemical or structural information about materials [94].

The following discussion of electron microscopy is by no means complete; the interested reader is referred to more detailed discussions in references [94-96]. Here, a few features of electron microscopy particularly salient to the studies in chapter 6 are presented.

\subsubsection{Bright-field and dark-field imaging}

When the electron beam in a microscope interacts with a crystalline sample, electrons can be elastically forward-scattered or diffracted. (Inelastic interactions occur as well; the reader is referred to $[94,95]$ for further information.) For bright-field imaging with 
a TEM, only the forward-scattered beam is collected for imaging purposes. This is the imaging mode with which many readers will be most familiar.

An alternative imaging mode that can be an extremely powerful tool for structural characterization is dark-field imaging. When examining a diffraction pattern, especially one originating from a mixture of materials, it is useful to know which parts of the material being imaged contributed to the different parts of the diffraction pattern. 


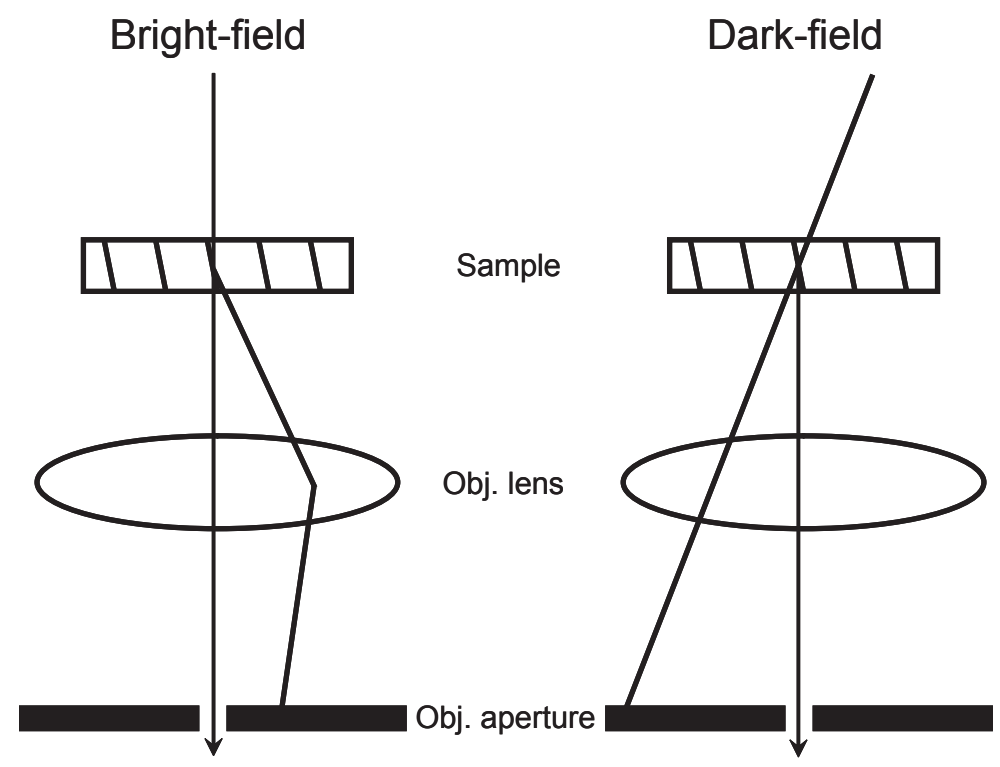

Figure 5.7. Basic beam alignment for bright-field imaging versus dark-field imaging. For both imaging modes, the objective aperture isolates electrons that form the image, blocking the rest. In bright-field mode, the main (forward-scattered) beam is allowed to travel along the optic axis. In dark-field mode, the incoming electron beam is tilted such that the diffracted beam is traveling along the optic axis. 

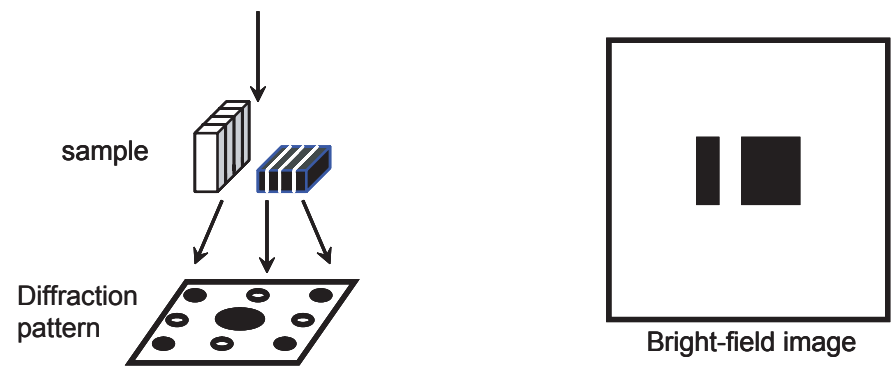

Bright-field image

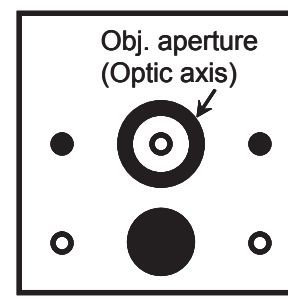

Pattern with tilted beam

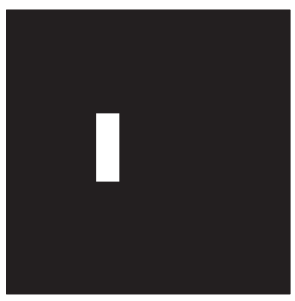

Dark-field image
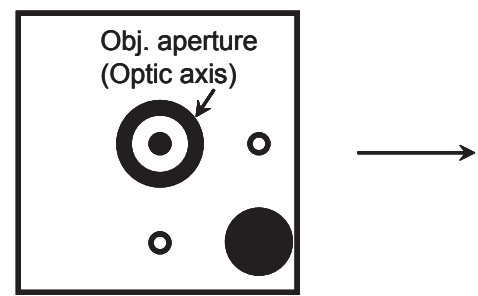

Pattern with tilted beam

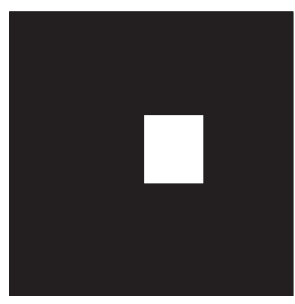

Dark-field image

Figure 5.8. Schematic of dark-field imaging. A sample consists of two crystals (colored black and white) with two orientations. (White diffraction spots correspond to the white crystal, black diffraction spots correspond to the black crystal.) In bright-field mode, the image is formed with the primary forward-scattered beam; hence the crystals appear dark, as part of the beam has been diffracted. In dark-field mode, the beam is tilted such that electrons from a given reflection travel along the optic axis. The part of the sample that contributes to this particular reflection "lights up" in the image. The rest of the field remains dark because it is not contributing to the given reflection. Different parts of the sample are illuminated depending upon which reflections are isolated with the objective aperture. 
Dark-field imaging enables us to do this by imaging only with the electrons from a specific scattering event (e.g. a single reflection spot) of the diffraction pattern. The electron gun source is tilted such that the diffracted beam of interest is centered in the column (figure 5.7). Thus, only the parts of the sample that contribute to that particular part of the diffraction appear in the image, per the schematic in figure 5.8.

\subsubsection{High resolution transmission electron microscopy (HRTEM)}

With HRTEM, we can image extremely small features in a material, even learning about the crystal structure of a material at the scale of a few nanometers. HRTEM differs from conventional TEM in that phase information is included in the image, rather than being discarded. In HRTEM, the objective aperture is left out so that diffracted beams are part of the signal being used to form images.
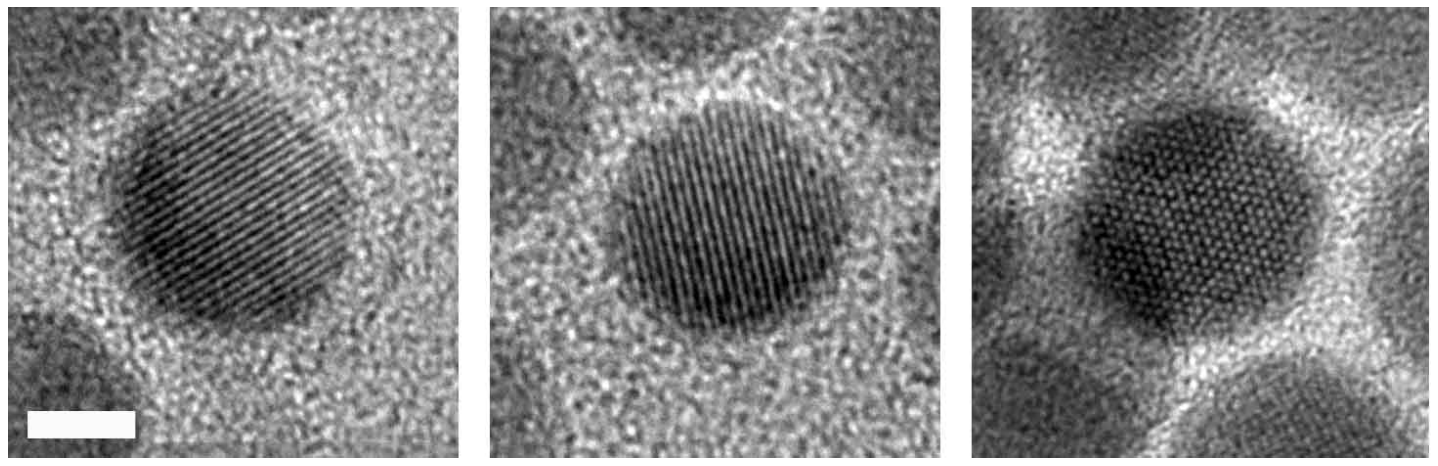

Figure 5.9. Typical HRTEM images of cobalt nanocrystals. Scalebar is $5 \mathrm{~nm}$. Images taken by D. Aruguete with the Alivisatos group TEM (FEI Tecnai 20). 
Figure 5.9 shows HRTEM images of cobalt nanocrystals. The periodic arrays of dots or lines are the result of constructive and destructive interference among scattered electron waves. The periodicities imaged will depend upon the orientation of the nanocrystal with respect to the beam, the quality of the illumination source, and the defocus setting of the objective lens. HRTEM thus can be used in conjunction with electron diffraction in a microscope to understand the finer crystallographic structure of a material. 


\section{CHAPTER 6. CRYSTALLOGRAPHIC CHARACTERIZATION OF $\varepsilon-$ COBALT NANOCRYSTAL ASSEMBLIES}

\subsection{Motivation and background}

Nanocrystals are not only a matter of academic study, but may also be of great importance for data storage and signaling applications [20,22]. With increasing miniaturization of computer data storage, there is great interest in controllably creating nanometer scale features for circuitry and other devices. One means by which nanoscale devices could be built is the "bottom up" approach, in which nano-sized or moelecular components assemble themselves into desired structures [23, 24, 99]. More recent work has suggested that such approaches could even be integrated with traditional "top down" device fabrication methods, such as lithography [99, 100].

When a solution of colloidal nanocrystals is allowed to dry on a substrate, the nanocrystals often spontaneously assemble to create ordered structures. Highly

monodisperse nanocrystals will often form lattice-based arrays, or "supercrystals" in which the nanocrystals themselves behave as "artificial atoms". Such behavior has been observed for many different types of colloidal nanocrystals, including various semiconductors (PbTe [101], CdSe [102] ) and metals (Au [103], Ag [104-106], FePt [107], Co [102, 108-110]). Recently, even ordered arrays of mixed nanocrystals (two different types of nanocrystals) have been shown to be possible $[27,111,112]$.

Magnetic nanocrystals and their assemblies are of particular interest for data storage or signaling because each nanocrystal contains a single magnetic domain which in theory can 
be manipulated [50]. In other words, each nanocrystal contains a single large magnetic dipole (the sum of the atomic magnetic dipoles acting collectively). Controlled assembly of magnetic nanocrystals could lead to such breakthroughs as a higher-density hard drive [107]. The magnetic properties of such nanocrystals are size-dependent [113] and can vary with surface modifications [114]. Thus with rigorously-controlled syntheses, the properties of the nanocrystals should be finely tunable.

Understanding the mechanisms of magnetic nanocrystal assembly can be complicated, as both electrostatic Van der Waals interactions and dipole interactions must be considered. Currently, there are two size regimes for which magnetic nanocrystal assembly is well-understood. Representative TEM images of these assemblies are featured in figure 6.1. Nanocrystals that are small enough are superparamagnetic, meaning that their magnetic dipoles are not aligned along any given crystal axis for a significant period of time [50]. These nanocrystals will assemble according to Van der Waals forces, approximately imitating hard-sphere packing models (with soft shells) [25], as shown in figure 6.1.1. For a larger nanocrystal, the magnetic dipole is greater and more strongly fixed along a given easy axis (or axes) of the nanocrystal [115]. Thus, interparticle dipole interactions are more important for larger nanocrystals, causing them to assemble into loops or chains (Fig 6.1.2.). These arrangements minimize the magneto-static energy of the dipole interactions.

One area of magnetic nanoparticle assembly that has not been so well-studied is the size regime in which both Van der Waals forces and magnetic forces play a key role. This chapter concerns the preparation and structural characterization of assemblies formed by particles in this size regime, namely $9 \mathrm{~nm}$ diameter cobalt nanocrystals. These nanocrystal assemblies were crystallographically aligned in a novel fashion. Characterizations of these 
assemblies with selected area electron diffraction (SAED) and complementary TEM imaging techniques are discussed in detail.
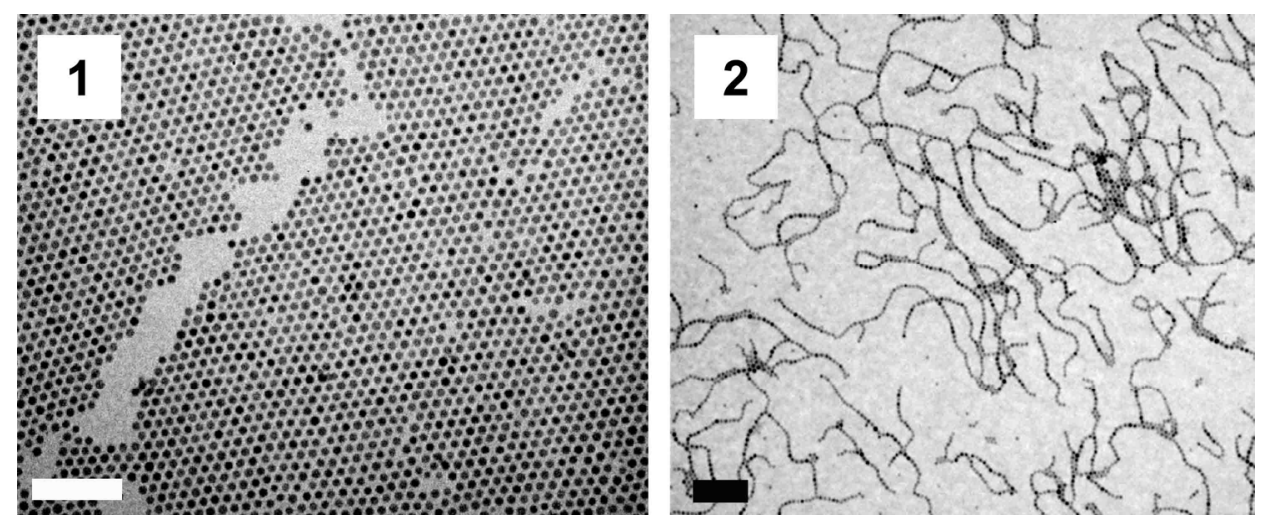

Figure 6.1. Both images are of cobalt nanoparticles ( $\varepsilon$-phase) after drying a solution on a TEM grid. (1) Cobalt nanoparticles $<9 \mathrm{~nm}$ in diameter. Magnetic interactions between them are insignificant, thus they display close-packing behavior dominated by Van der Waals forces. Scalebar is $100 \mathrm{~nm}$. (2) Cobalt nanoparticles $\sim 16 \mathrm{~nm}$ in diameter. Stronger magnetic forces cause the particles to form loops or chains. Scalebar is $200 \mathrm{~nm}$. Images were collected at the UCB Electron Microscope Laboratory with a Philips Tecnai 12 by D. Aruguete.

\subsection{Preparation of $\varepsilon$-cobalt nanocrystals and their assemblies}

All synthesis and processing of the cobalt nanocrystals were performed under airfree, water-free conditions. $9 \mathrm{~nm}$ diameter cobalt nanocrystals were synthesized via previously established methods [116] that will be briefly described here. To summarize, dicobaltoctacarbonyl (dissolved in 1,2 dichlorobenzene, or DCB) was rapidly injected into a boiling solution of oleic acid and trioctylphosphine oxide (TOPO) in 1,2 DCB. Aliquots (in nitrogen-purged vials) were collected 1, 3, and 5 minutes after injection and immediately 
transferred into an argon drybox. The nanocrystals were cleaned with a single step of sizeselective precipitation ( 1 part methanol to 3 parts solution) to improve the size distribution; the remaining precipitate was re-suspended in 1,2 DCB and stored at room temperature in the drybox. No further washing was performed; thus it is likely that this suspension contained some residual methanol, oleic acid, and TOPO. X-ray diffraction confirmed that the samples were $\varepsilon$-cobalt, a phase of cobalt that has only been found in nanocrystals [117]. $\varepsilon$-Co is isostructural with a high-temperature phase of manganese $(\beta-\mathrm{Mn})$, and is a cubic, non close-packed structure.

All TEM samples were made and stored under air-free, water-free conditions. A schematic of sample preparation is depicted in figure 6.2. Samples were made by depositing a drop $(5 \mu \mathrm{L})$ of the concentrated suspension onto an amorphous carbon-coated transmission TEM grid on top of a paper towel, which absorbed excess solution. The grids were then covered and allowed to dry. (While initially they were allowed to dry for a minimum time of 2 hours, later observations showed that the substrates generally dried in 510 minutes.) This resulted in the formation of close-packed multilayered nanocrystal islands (Figure 6.3), as well as discontinuous monolayers. Interestingly enough, the choice of substrate did not make a clear difference in the formation of the islands; amorphous carbon, plasma-treated amorphous carbon (hence charged, and more polar), and silicon monoxide could be used. 

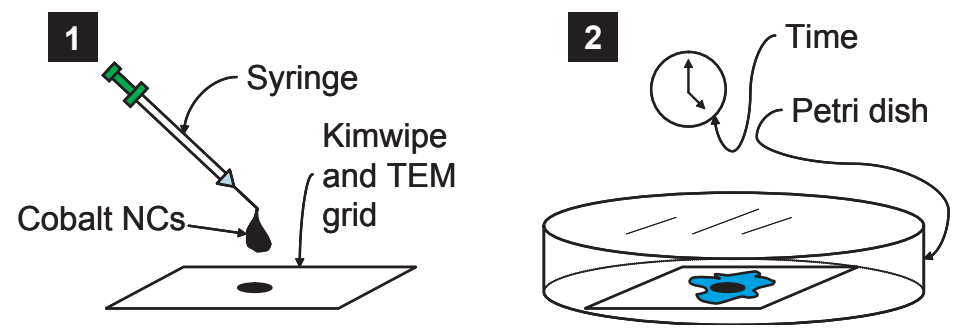

Figure 6.2. Preparation of cobalt nanocrystal assemblies on TEM grids.

Excess surfactant on nanocrystals can create problems for TEM imaging by not effectively conducting away charge. Thus, many of the TEM samples were additionally washed by gently depositing drops of methanol directly onto the grids and wicking away the excess. Unfortunately, this had the consequence of making the samples more susceptible to oxidation, even within the time it took to transfer the sample from an air-tight vial to the electron microscope vacuum column. In other samples, oxidation was avoided by simply not washing the TEM grids.

\subsection{Transmission electron microscopy (TEM) and SAED (selected-area electron diffraction) of cobalt nanocrystal assemblies}

\subsubsection{Instrumentation}

Transmission electron micrsocopy (TEM) and selected area electron diffraction (SAED) were used to structurally characterize the assemblies formed from deposition. TEM and SAED were conducted utilizing a number of different microscopes at UC Berkeley and the National Center for Electron Microscopy (NCEM) at Lawrence Berkeley National Laboratory (LBNL). The UC Berkeley microscopes used were an FEI Tecnai 12 at the UCB 
Electron Microscope Laboratory (EML) and an FEI Tecnai 20 in the Alivisatos group. The electron diffraction and dark field imaging were performed primarily at NCEM using the JEOL 3010 in-situ microscope and the JEOL 200CX analytical electron microscope. The Philips CM 200, Philips CM 300, JEOL-JEM ARM 1000, and the Topcon 002B microscopes were also used for high-resolution imaging, conventional imaging, and diffraction.

\subsubsection{TEM image/SAED pattern processing}

All images and patterns were subjected to the minimum levels of digital processing deemed necessary for obtaining useful data. For most images, the tonal range (the gray levels) was adjusted using the Levels command in Adobe Photoshop 7.0. Occasionally, slight corrections to the contrast and brightness were also made in Adobe Photoshop 7.0 using the Brightness/Contrast command. The most heavily-processed images were some of the dark field images, which had their tonal ranges adjusted such that most or all of the gray levels were discarded; these images are shown in figures 6.12 and 6.13. Examples of adjustments to tonal ranges with Adobe Photoshop 7.0 are in Appendix 1. Fourier transforms of images were performed using Media Cybernetics Image Pro Plus version 4.5.0.19.

All of the SAED patterns analyzed had been originally captured on film. Many of the $\varepsilon$-Co reflections important for analysis are very closely spaced, necessitating the high resolution and dynamic range of film. Negatives were scanned at NCEM and in the Alivisatos group computer laboratory at the highest resolution possible (often at $1200 \mathrm{dpi}$ ) as 16-bit tagged image files (tifs). 


\subsubsection{Bright-field imaging of the assemblies}

The structures formed from the deposition of the nanoparticles were generally closepacked multilayered nanocrystal islands, as well as discontinuous monolayers (figure 6.3).
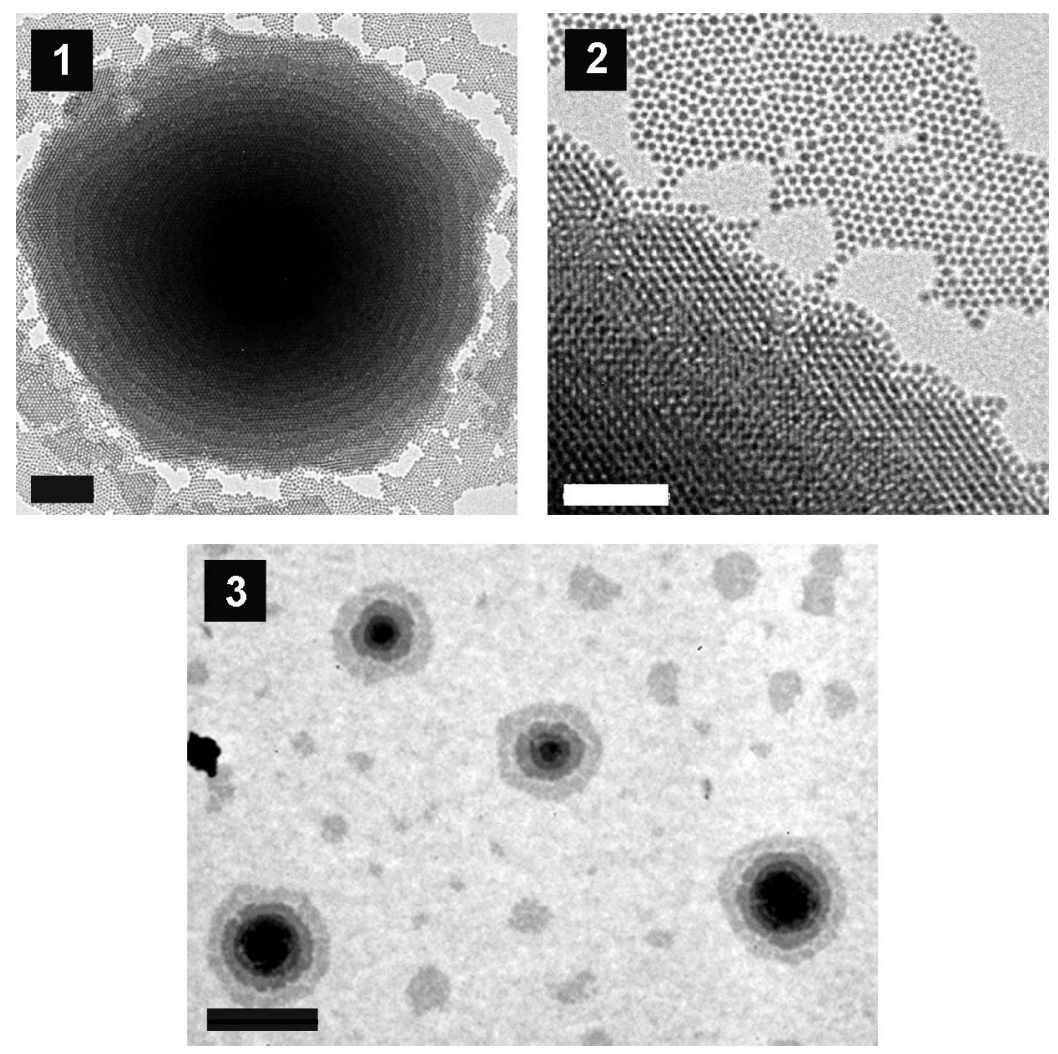

Figure 6.3. Examples of structures formed after deposition of cobalt nanocrystals. (1)

Multilayered island of close-packed nanocrystals surrounded by patches of mono- or bilayers. Scalebar is $0.2 \mu \mathrm{m}$. (2) Magnification of upper right-hand corner of image (1), showing closepacked layers of nanocrystals in island. Scalebar is $100 \mathrm{~nm}$. (3) More multilayered islands, surrounded by unconnected monolayers. Scalebar is $2 \mu \mathrm{m}$. Images (1) and (2) were collected on the JEOL 3010 at NCEM by Dr. Radetic; image (3) was collected at the UCB EML by D. Aruguete. 


\subsubsection{SAED from monolayer and multilayer assemblies}

The monolayers exhibited electron diffraction patterns typical of polycrystalline films with no preferred crystallographic orientations (figure 6.4). Indexing the diffraction pattern reveals that the nanocrystals are cubic $\varepsilon$-cobalt, in agreement with XRD measurements.
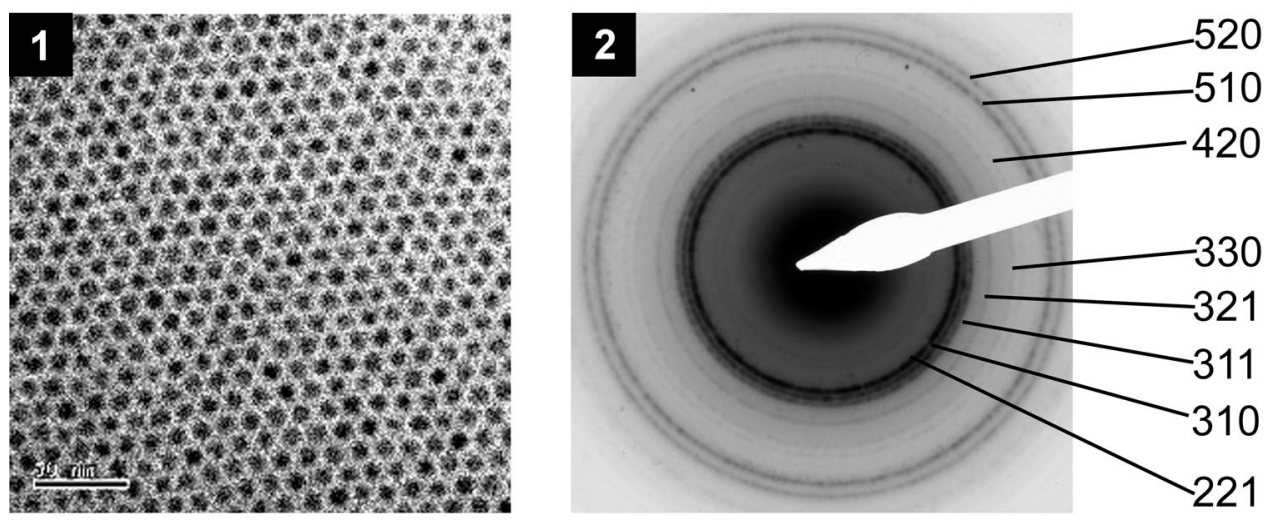

Figure 6.4. (1) Part of a monolayer of cobalt nanocrystals. Scalebar is $50 \mathrm{~nm}$. (2) Typical SAED pattern from a monolayer. Indexing reveals that the nanocrystals are cubic $\varepsilon$-cobalt. Image and SAED were collected by D. Aruguete at the UCB EML.

SAED from islands (typically containing 10-15 layers, but some with as few as 4 layers), on the other hand, commonly displayed concentration of diffracted beams into strong spots, as well as the extinction of some reflections. There were two types of patterns generally observed, both of which generally had a six-fold symmetric pattern. Examples of the most common type of pattern observed are shown in figure 6.5; indexing is shown in figure 6.6. This pattern displays a kinematically forbidden 200 reflection which cannot be attributed to oxides or other cobalt phases. This 200 reflection may be the result of various dynamical events due to a thin film of oxide on the nanocrystal surfaces, or phenomena such as double 
diffraction $[94,95]$. A second type of diffraction pattern was observed in one instance, shown in figure 6.7. It is clear from the indexed reflections that the patterns in 6.6 and 6.7 are different. Details of indexing for all wide-angle SAED patterns are documented in section 6.4 and Appendix 2.

This strong six-fold symmetric texture was observed in SAED patterns from the centers of the islands, as shown in figure 6.8. From probing different parts of the islands (sides versus centers) it was evident that the preferred orientations were not maintained throughout the island. 

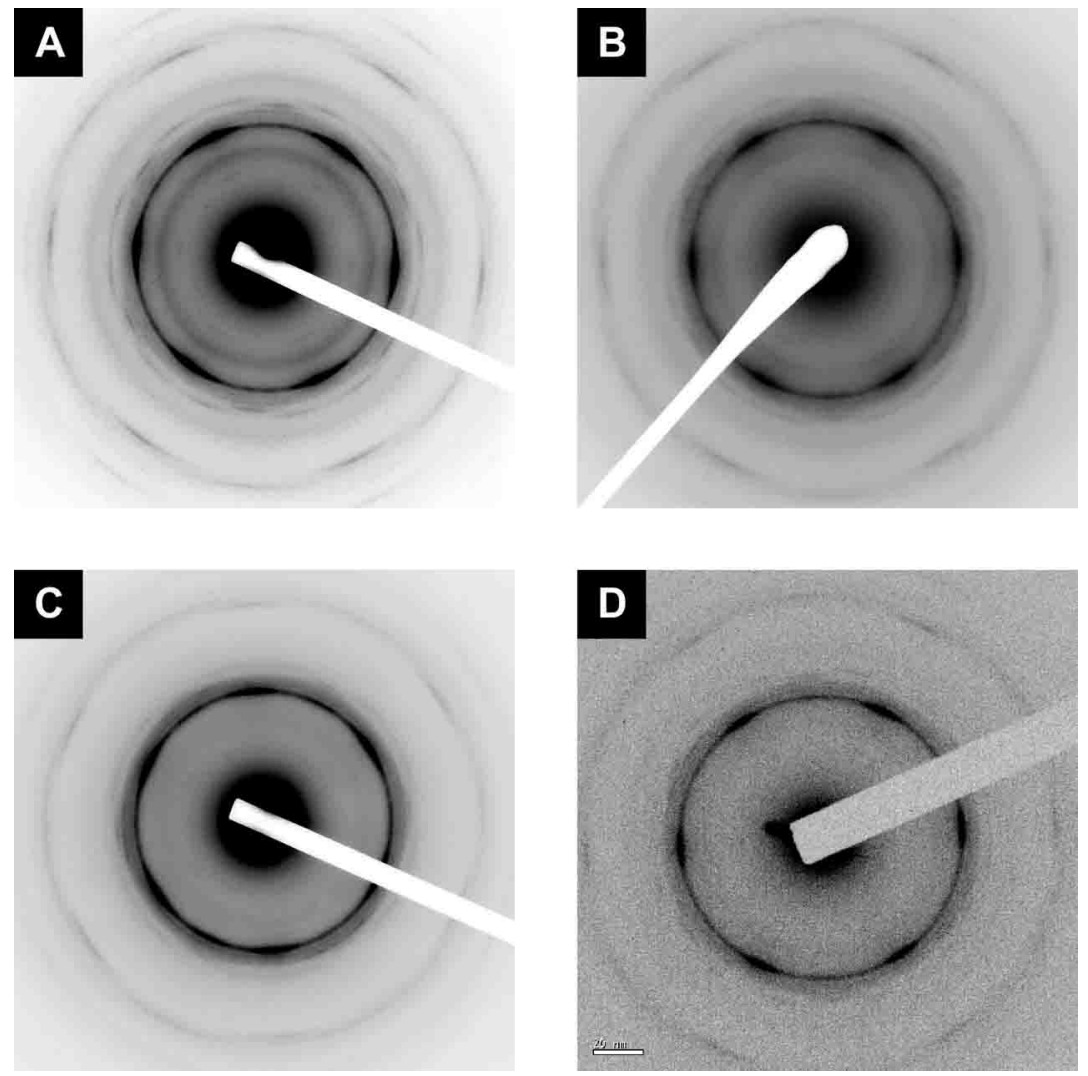

Figure 6.5. SAED patterns from various multilayered islands. Patterns were all collected at NCEM with the JEOL 3010 and the Topcon 002B by D. Aruguete, except for the lower right-hand corner pattern collected by Dr. Radetic. 


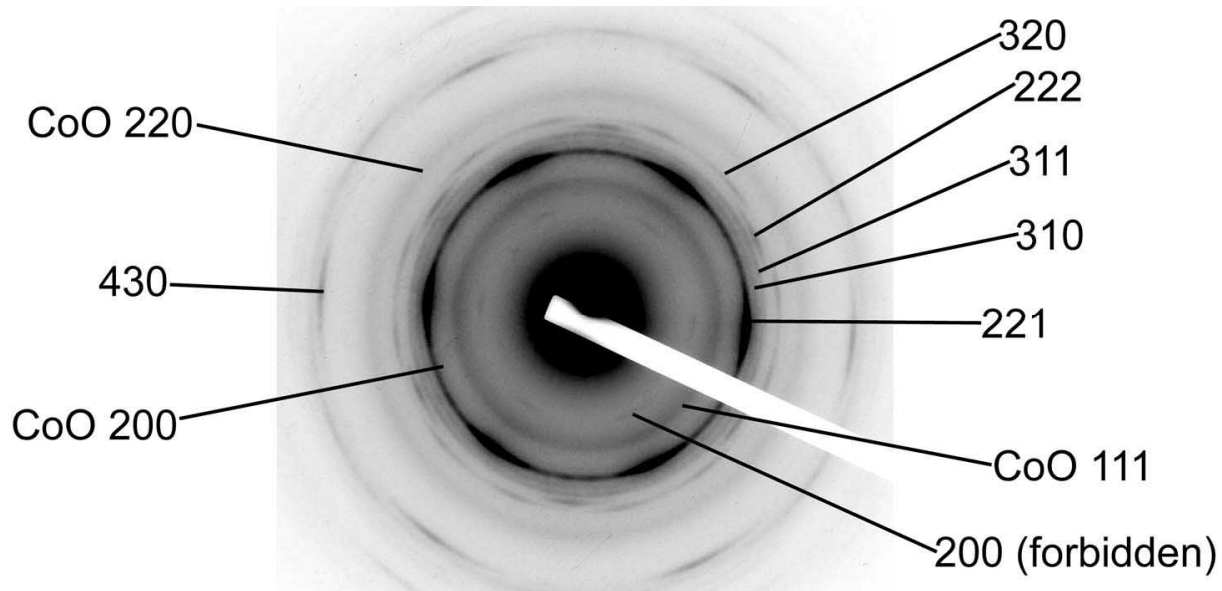

Figure 6.6. Typical SAED pattern from the center of a multilayer island, with indexing. The 221 reflection is the most intense and is strongly textured. Note the presence of the kinematically forbidden 200 reflection. Pattern collected by D. Aruguete using the JEOL 3010 at NCEM.

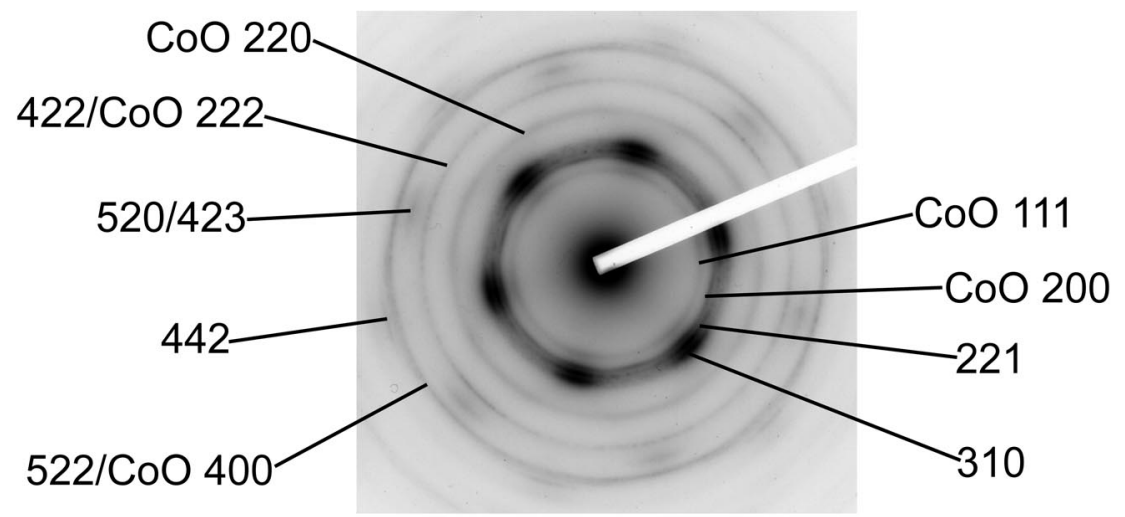

Figure 6.7. Alternate SAED pattern from multilayer island, with indexing. Note how this pattern differs from the more common textured pattern in figures 6.5 and 6.6. The 422 and $522 \varepsilon$-cobalt reflections coincide with those expected from $\mathrm{CoO}$; hence, they cannot be positively identified. Pattern collected by Dr. Radetic using the JEOL 3010 at NCEM. 

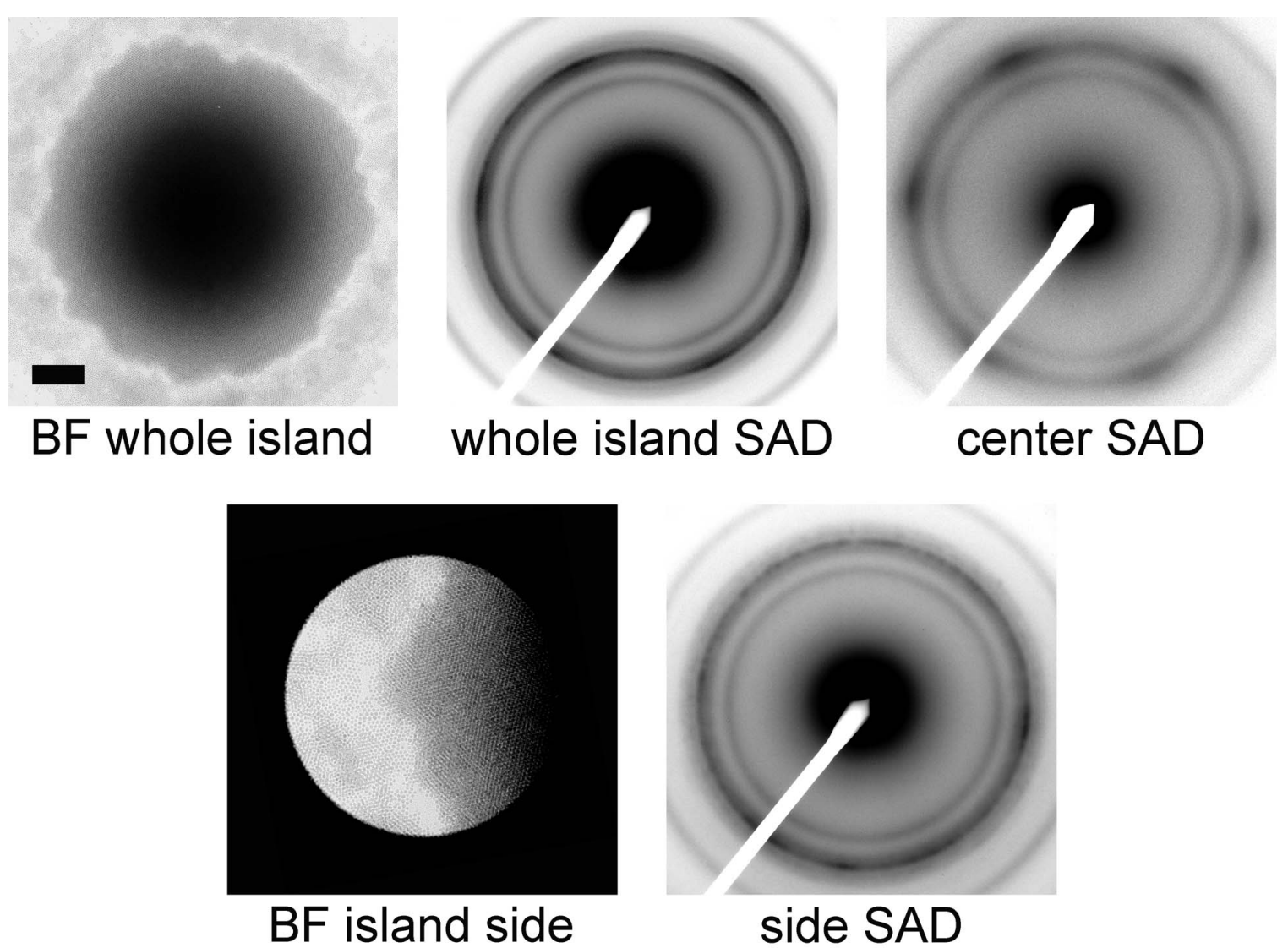

Figure 6.8. SAED from various parts of an island, approximately $1 \mu \mathrm{m}$ in diameter. SAED patterns from the center show that nanocrystals in the center of the island have preferred orientations. The SAED pattern collected from the side of the island does not show much preferred orientation. Scalebar in bright field image is $0.2 \mu \mathrm{m}$. Data collected by Dr. Radetic at NCEM, using the JEOL 200CX AEM. 
In addition to examining the crystallographic ordering of the atomic lattices within the nanocrystals, the structure of the lattice-based arrays of the nanocrystals (often incorrectly referred to in the general literature as "superlattices" [103]) was inspected using small-angle electron diffraction. Small-angle electron diffraction was performed by increasing the camera length in the microscope (from 1.6-3 m) and using short (often $<1 \mathrm{~s}$ ) exposure times. These three-dimensional lattice-based arrays were generally found to have a face-centered cubic (fcc) structure, often with a $<111>$-type zone axis. Representative patterns from an island are shown in figure 6.9 $1 \mathrm{~A}$ and $1 \mathrm{~B}$. In $1 \mathrm{~A}$, a pattern is shown that appears to be originating from an fcc crystal with a $<111>$-type zone axis. To confirm that the nanocrystal lattice had fcc packing, the island was tilted; at a $30^{\circ}$ tilt, the expected pattern from an fcc crystal with a $<110>$-type zone axis was observed (the angle between these vectors is $\left.35.26^{\circ}\right)$. Occasionally, other zone axes such as $<110>$ were found for non-tilted samples (Fig. 6.9.2). There was no immediately obvious correlation between the orientations of these arrays and the atomic lattice orientations, although further study is merited. 

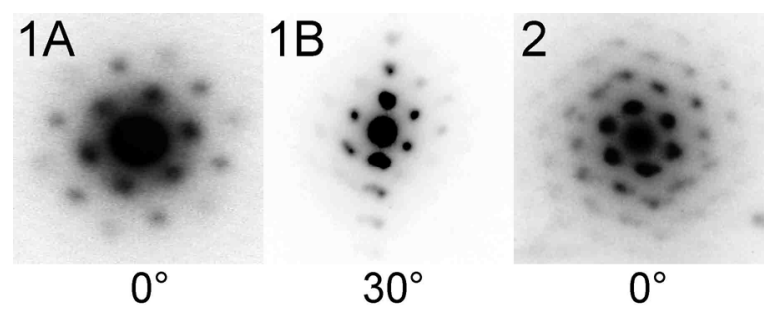

Figure 6.9. Small-angle diffraction from the lattice-based arrays of nanocrystals present in the islands (Camera length $=3 \mathrm{~m}$ for patterns $1 \mathrm{~A}$ and $1 \mathrm{~B}, 1.6 \mathrm{~m}$ for pattern 2 ). Patterns shown in $1 \mathrm{~A}$ and $1 \mathrm{~B}$ arise from the same crystal. $\mathrm{A}$ is the SAED from an fcc crystal with a $<111>$-type zone axis, while B shows the same crystal tilted, such that it is oriented with a $<110>$-type zone axis. (2) Example of a non-<111>-type zone axis from another island; even at zero tilt, this island produces a pattern typical of a $<110>$-type zone axis orientation for an fcc crystal. Patterns $1 \mathrm{~A}$ and 1B collected at NCEM on the JEOL 3010 by Dr. Radetic and D. Aruguete. Pattern 2 collected at NCEM on the Topcon 002B by D. Aruguete.

\subsubsection{Dark-field imaging from multilayered assemblies}

Dark-field images were collected to determine if there were any higher levels of organization present in the assemblies. In figure 6.11.A-1, A-2, and A-3, dark-field imaging using the arcs present in the 221 reflections indicated that three separate groups of nanocrystals were contributing to these reflections. (The 310 reflections could not be excluded from the dark-field imaging, as the objective aperture was not small enough.) Of particular interest was that when the $\vec{g}_{221}$ corresponding to the intense arcs or spots in the 221 reflection are assigned to the various domains, a loop-closing arrangement is suggested. (Remember that the $\varepsilon$-Co phase is cubic; hence real-space and reciprocal-space vectors with the same indices hkl point in the same direction. This shall be further discussed later in this 
chapter.). The images in figure 6.3 , as well as those in figure 6.10 suggest that nanocrystal monolayers with this loop-closing arrangement are stacked on top of each other to form a multilayered island with a vortex-like alignment of the nanocrystals (figure 6.11). Loopclosing structures may originate from magnetic interactions between the nanocrystals [118]. This topic will be further discussed in section 6.6.1.1.

Dark-field images were also collected from samples with the more common type of six-fold symmetric pattern, shown in figures 6.12 and 6.13 . While these images are faintly suggestive of the sort of arrangement displayed in figure 6.8, this can only be seen when most of the gray levels were removed from the tonal range of the image (details in Appendix 1). Thus, it is not certain that the same phenomenon of higher ordering is occurring within these assemblies. 

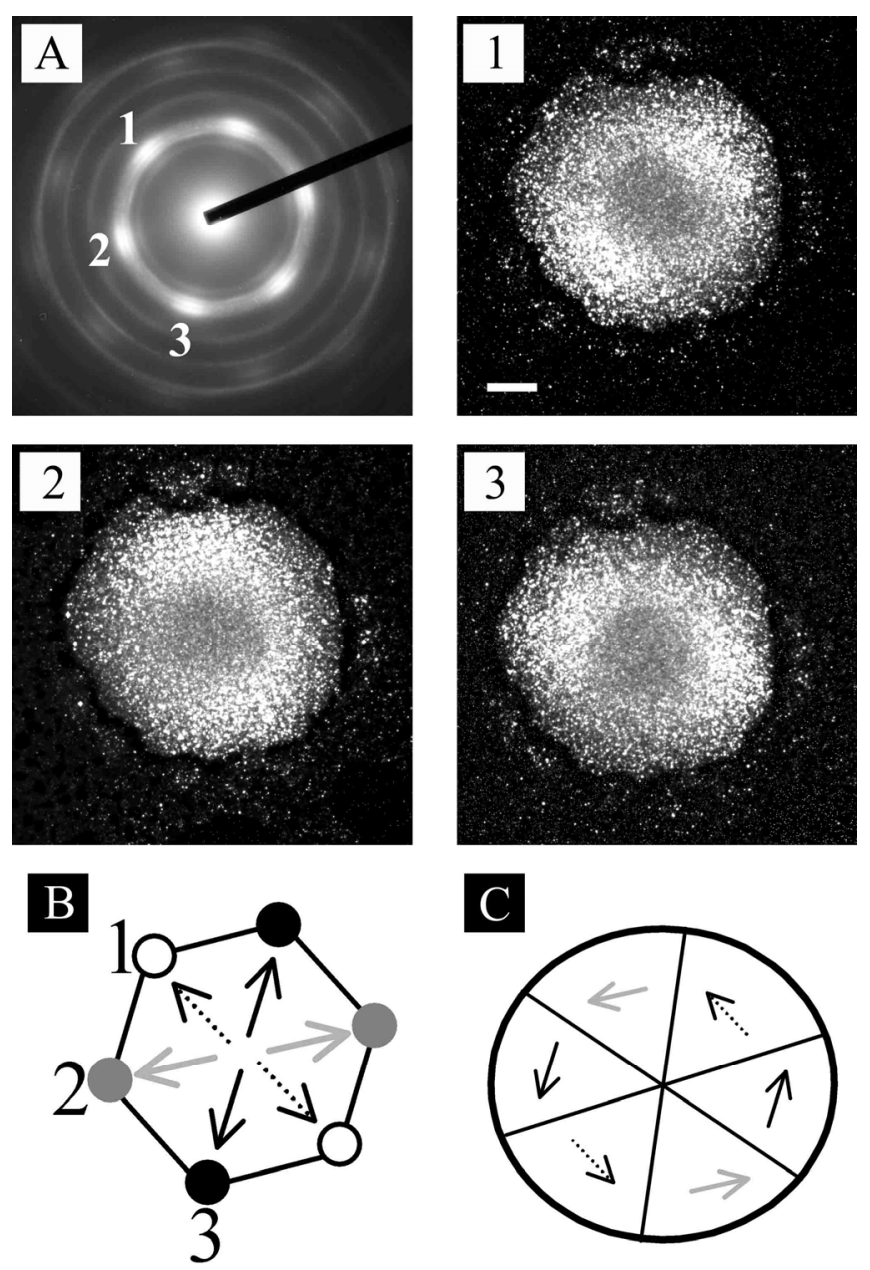

Figure 6.10. (A) SAED pattern shown in figure 6.7 multilayered island. Images (1), (2), and (3) are dark-field images taken from the correspondingly labeled reflections in image $\mathrm{A}$. The objective aperture was not small enough to separate the 221 and 310 reflections. (B) Schematic of $\vec{g}_{221}$ from the SAED pattern in (A). (C) When these vectors are assigned to the different illuminated segments of the dark-field images corresponding to these reflections, an interesting loop-closing arrangement is suggested. Scalebar is $0.2 \mu \mathrm{m}$. Data was collected at NCEM from the JEOL 3010 by Dr. Radetic. 


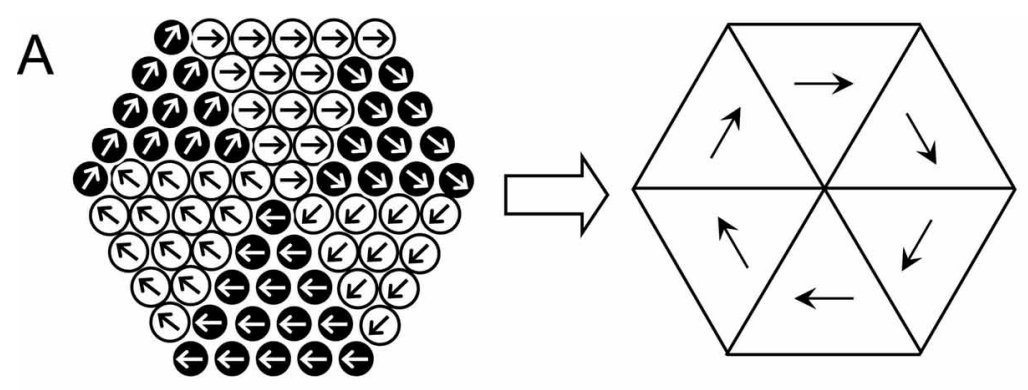

A layer of close-packed cobalt nanocrystals in an island

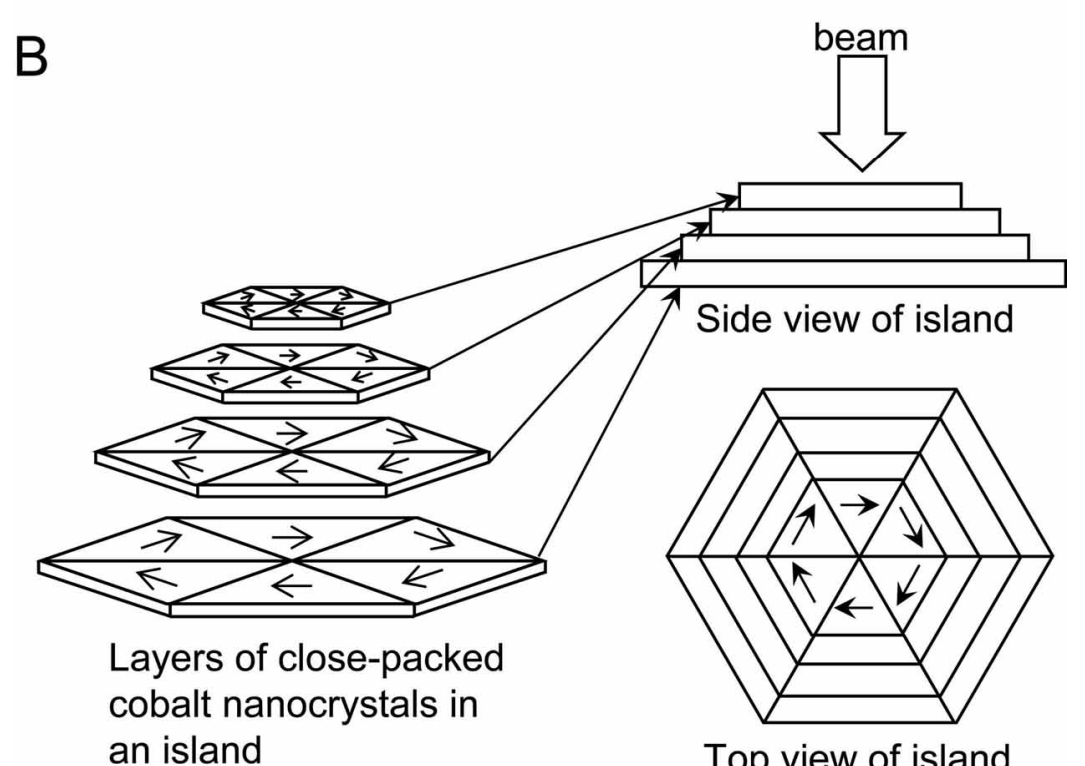
an island

Top view of island

Figure 6.11. Schematic of possible crystallographic orientations of the nanocrystals within an island. (A) A single layer of close-packed nanocrystals, constituting a layer of the island. Three crystallographic orientations are present. This monolayer is represented as a hexagonal plate with three crystallographic orientations. (B) Stacks of these monolayers constitute a multilayered island with a 3D vortex-like orientation of the nanocrystals. 

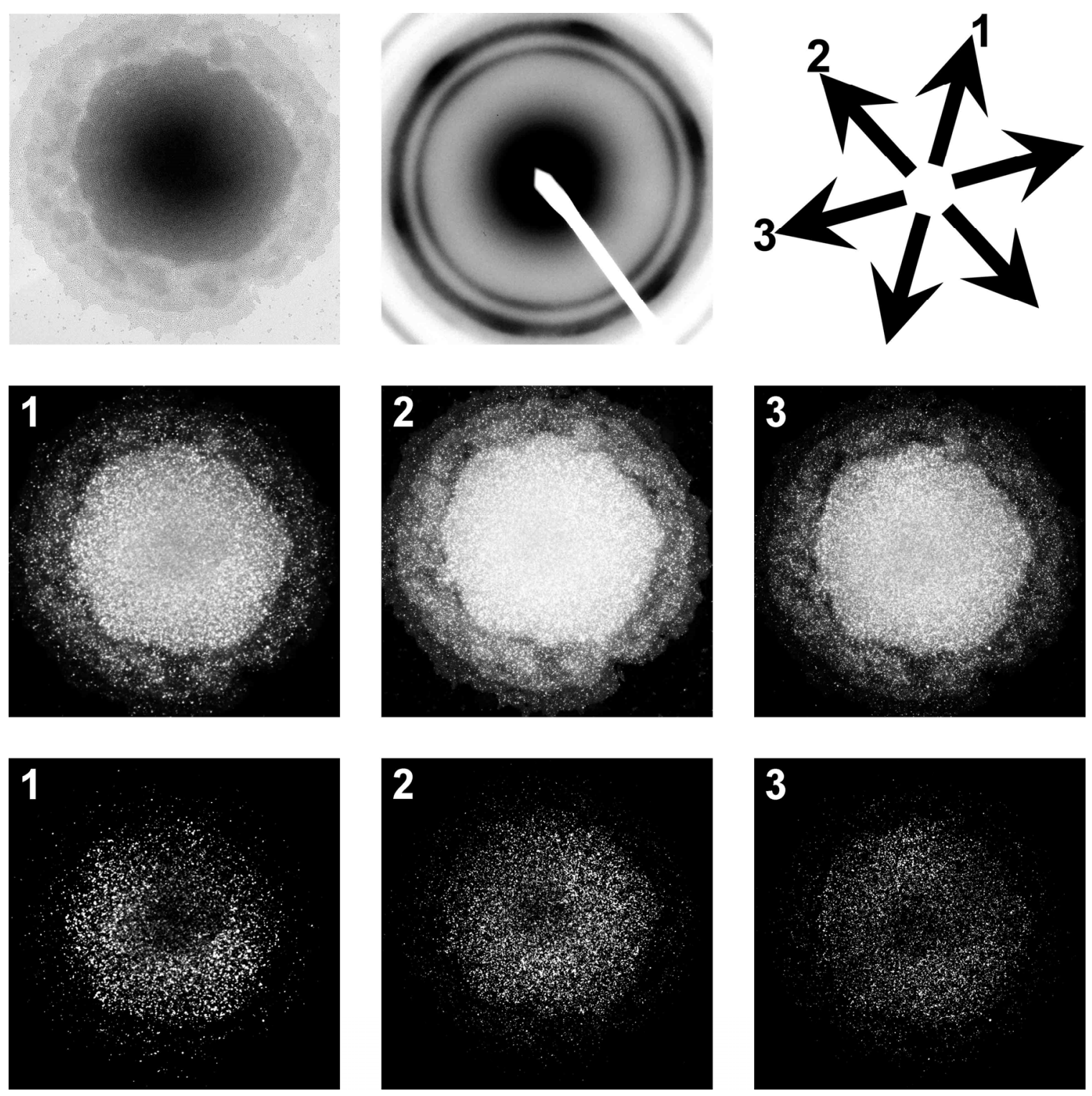

Figure 6.12. Top row, left to right: Bright field image of an island approximately $1.5 \mu \mathrm{m}$ in diameter, SAED from the island, and a schematic of $\vec{g}_{221}$ corresponding to the observed arcs. Middle row, left to right: corresponding dark field images from reflections at positions one, two, and three. Bottom row, left to right: Dark field images with additional processing (described in section 6.3.2.) from reflections at positions one, two, and three. Note that these are suggestive of the same vortex-like configuration presented in figure 6.8. 

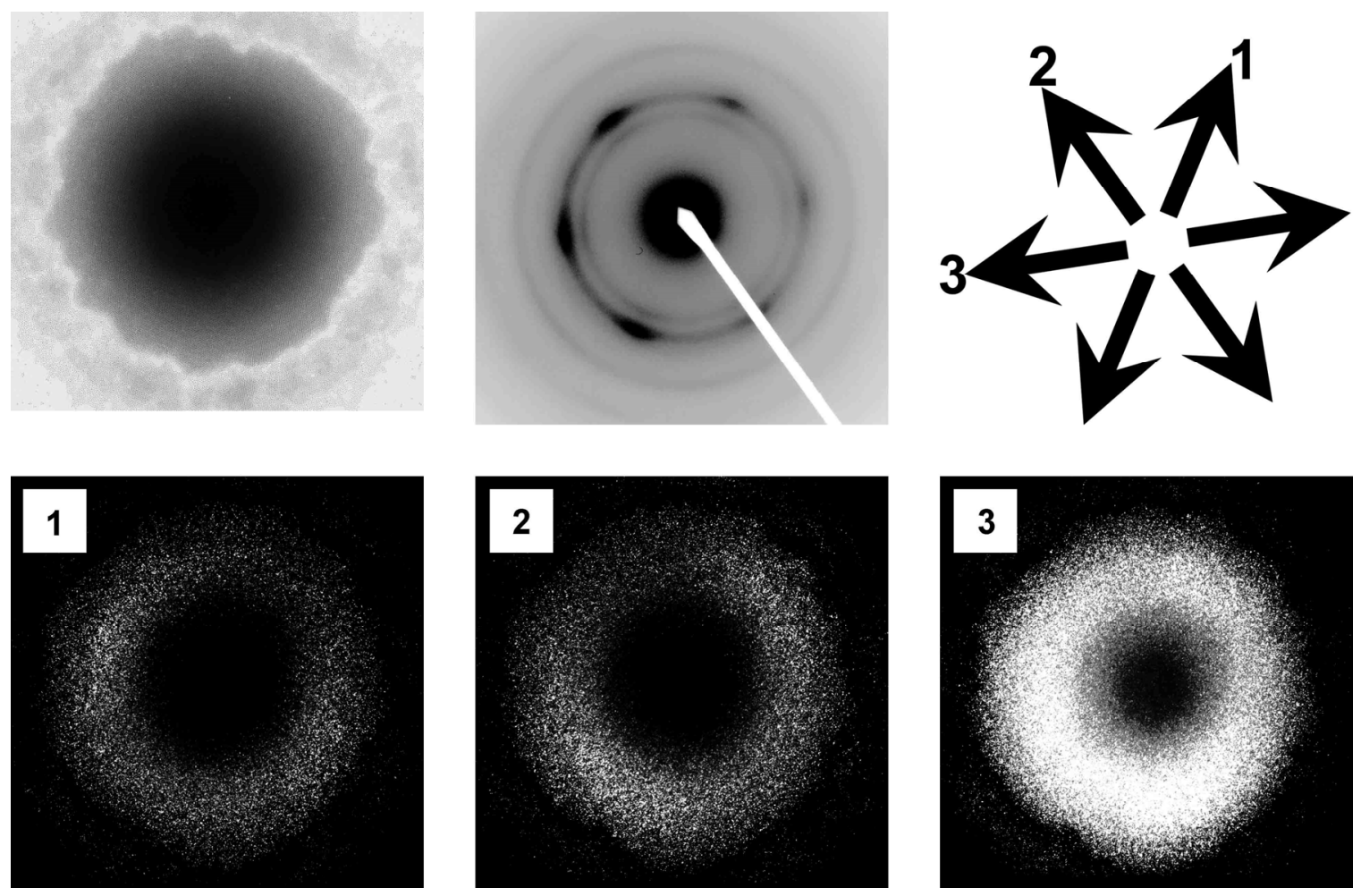

Figure 6.13. Another example of dark-field data from a multilayered island. Top row, left to right: Bright-field image of an island approximately $1 \mu \mathrm{m}$ in diameter, the corresponding diffraction pattern, and a schematic of $\vec{g}_{221}$ corresponding to the intense 221 arcs in the pattern. Bottom row, left to right: processed dark field images (see section 6.3.2. for description of image processing) corresponding to those 221 reflections labeled in the schematic. Note that while for images (1) and (2), the corresponding $\vec{g}_{221}$ are aligned in a vortex-like fashion similar to that present in figure $6.8,(3)$ is not particularly consistent with this model. 


\subsubsection{High-resolution TEM (HRTEM) imaging of individual particles and their assemblies}

\subsubsection{HRTEM of individual particles}

When attempting to understand the factors influencing the assembly of nanocrystals, it is important to consider their individual shapes. The presence of their surfactant coatings does not rule out the effect of shape upon assembly ; indeed, in previous studies, surfactantcoated faceted nanocrystals were seen to pack in a limited number of orientations, giving rise to textured diffraction patterns [102, 103].

To closely examine the morphology of individual $\varepsilon$-cobalt nanocrystals, they were imaged with HRTEM. HRTEM data was collected by D. Aruguete as well as Steven Hughes, a fellow graduate student in the Alivisatos group. Representative mages of the nanocrystals are shown in figure 6.14. While such images cannot rule out the presence of facets, it appears that these nanocrystals do not have pronounced faceting.
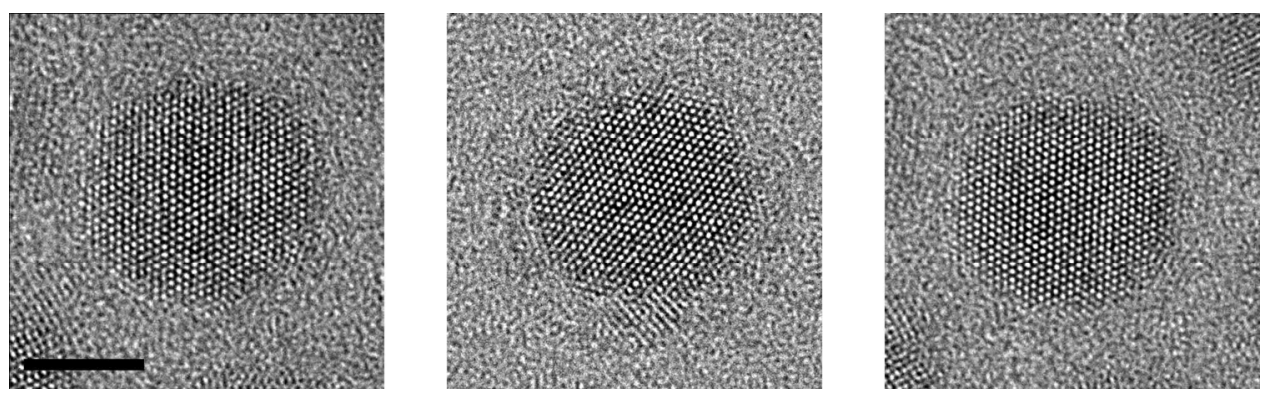

Figure 6.14. HRTEM images of three separate $\varepsilon$-cobalt nanocrystals. Scalebar is $5 \mathrm{~nm}$. The nanoparticles were imaged at NCEM by Steven Hughes, using the Philips CM300. 


\subsubsection{HRTEM of multilayer nanocrystal assemblies}

HRTEM imaging was used to further examine the local structure of nanocrystal orientation within the assemblies. HRTEM of these assemblies was complicated by multiple factors. First, the beam sometimes induced what was likely to be carbonization of the surfactants coating the nanocrystals; in minutes, a film formed upon the imaged areas, occluding any lattice fringes. Second, it was difficult to maintain proper stigmation; the nanocrystals are magnetic and hence local magnetic fields vary depending upon the position of the beam on the sample. Third, the most highly aligned parts of the islands are the centers, which are the thickest parts of the islands. Repeated attempts to image through these centers, even with high-energy electrons (800-1000 keV) proved to be unsuccessful; not enough electrons were transmitted to provide any signal. (Note that for hcp Co, the extinction distances of $1000 \mathrm{keV}$ electrons range from $42.5 \mathrm{~nm}$ to $208.6 \mathrm{~nm}$ [95].)

The few partially successful images that were obtained are displayed in figures 6.15 and 6.16. Sections of images were Fourier-transformed with Media Cybernetics Image Pro Plus version 4.5.0.19 to look for any preferred orientations. Some preferred orientation was seen on the edge of an island, as shown in figure 6.15.3. Other results could not be clearly interpreted; while suggestions of orientation were observed in the Fourier transform of the image (Fig. 6.16.2), such results could be artifacts due to sample drift, astigmatism, or beam alignment. 

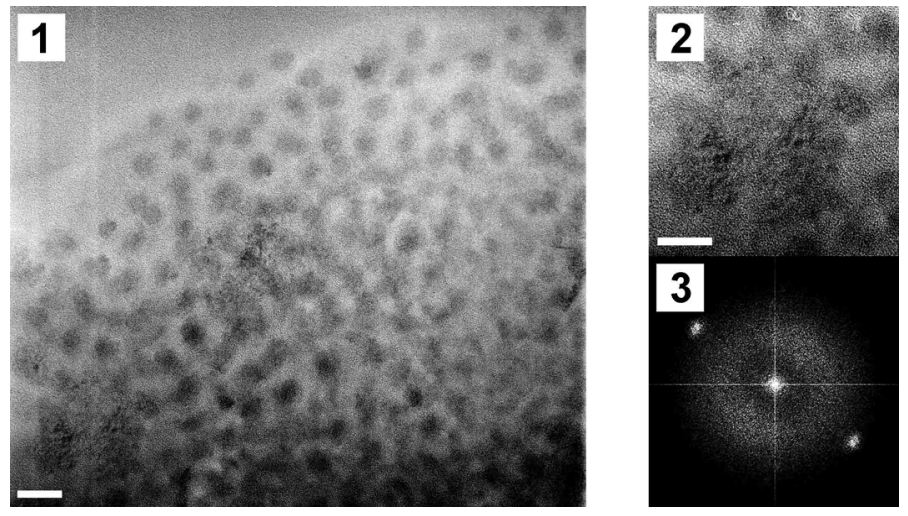

Figure 6.15. Example of partial orientation on edge of island. (1) is an image of an island edge. (2) is an inset from the lower left-hand corner of the image. (3) is a Fourier transform of this inset image, clearly showing a single strong frequency with a single orientation. Scalebars are $10 \mathrm{~nm}$. Images were collected by D. Aruguete from the NCEM JEOL-JEM ARM 1000 at 800-1000 keV.
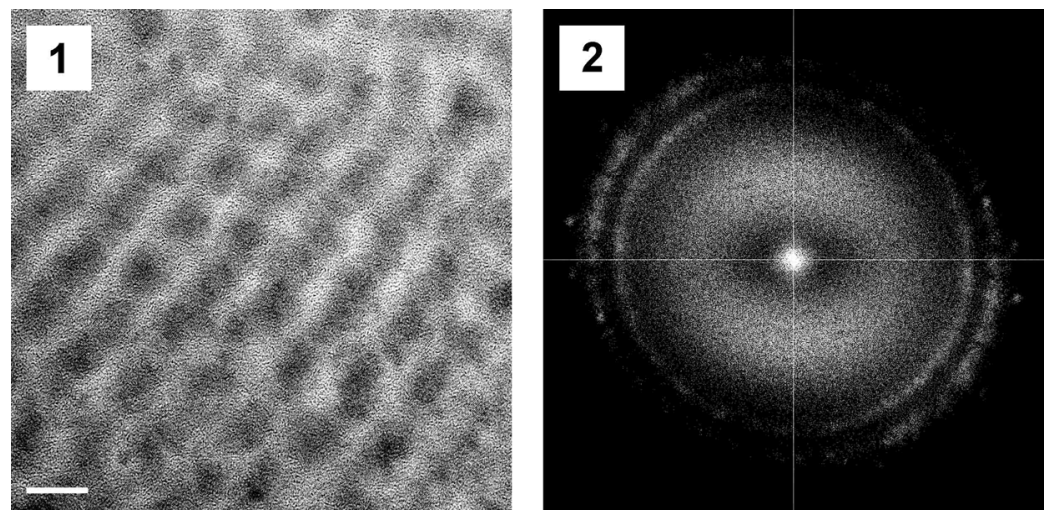

Figure 6.16. (1) Another HRTEM image from an island. (2) The corresponding Fourier transform. Scalebar is $10 \mathrm{~nm}$. Image collected by D.Aruguete from the NCEM JEOL-JEM ARM 1000 at 800$1000 \mathrm{keV}$. 


\subsection{Basic interpretation of SAED patterns}

When faced with any diffraction pattern, the first step in interpretation is to index the various reflections (identify which planes contribute to which reflections). As we already know that the substance is $\varepsilon$-cobalt from X-ray diffraction, this is fairly simple. Electrons are scattered by an angle $2 \theta$. It can be deduced via simple geometry that [94]

$$
\tan 2 \theta=\frac{r}{L}
$$

where $r$ is the radial distance from the center of the diffraction pattern to the reflection spot (or arc, or ring) of interest, and $L$ is the sample-to-detector distance.

As the scattering angle is quite small,

$$
2 \theta \cong \frac{r}{L} \text {, and }
$$

Bragg's Law reduces to

$$
\lambda=2 d \theta
$$

Combining equations 6.2 and 6.3 , we obtain the expression

$$
r d=\lambda L
$$

As $\lambda L$ is a constant term (known as the camera constant), this means that for any given set of reflections on a pattern,

$$
r_{1} d_{1}=r_{2} d_{2}=\ldots
$$

By simply measuring the distances $r$ on a pattern and comparing these ratios to $d$-spacing ratios, the pattern can be indexed.

Once the pattern is indexed, the next step is to figure out the real-space zone axis $[U V W]$, which is the crystallographic orientation of the electron beam with respect to the 
sample. The zone axis is common to all planes of the zone, that is, all planes that diffract for a particular orientation, and is perpendicular to the normal vectors of these planes, as depicted in figure 6.17. In other words, $U b+V k+W l=0$. This is known as the Weiss zone law [94].
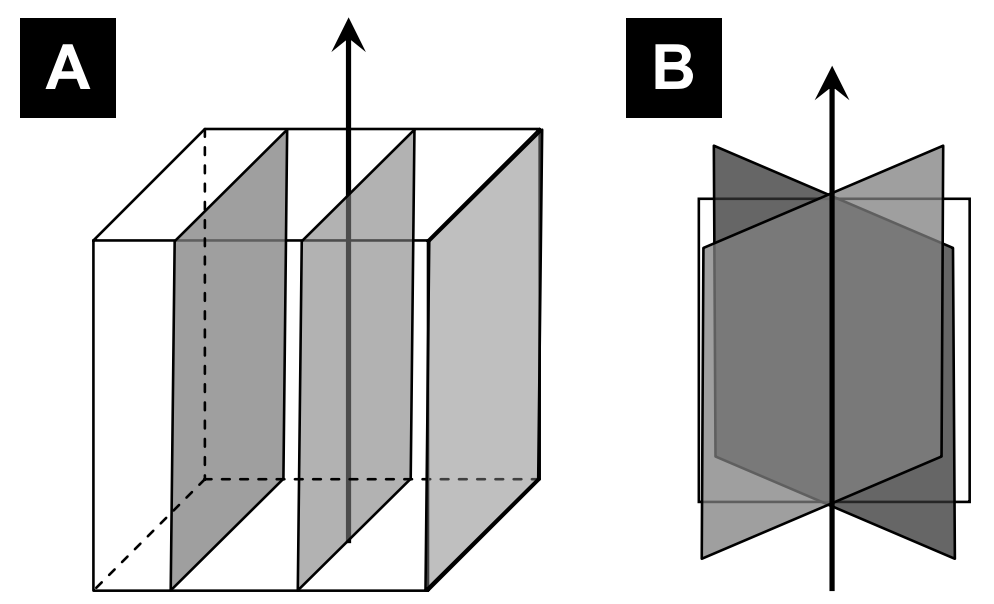

Figure 6.17. Two depictions of the zone axis for a crystal. In (A), a schematic of planes in a crystal is depicted, with the arrow as the zone axis (the direction of the incident electron beam). (B) shows how many planes in the crystal can contain the zone axis; by taking the cross product of the corresponding reciprocal space vectors for these planes, one can obtain the zone axis.

It may seem impossible for diffraction to occur for planes containing the zone axis (the incident beam direction vector); in the Bragg equation, certainly, diffraction cannot occur for $\theta=0^{\circ}$. The Laue conditions for diffraction (Eqn. 5.8) stipulate that

$$
\vec{k}_{I}-\vec{k}_{F}=\vec{g}_{h k l},
$$

where $\vec{k}_{I}$ is the incident electron wavevector, $\vec{k}_{F}$ is the scattered electron wavevector, and $\vec{g}_{h k l}$ is a vector in reciprocal space corresponding to the (bke) planes in the real crystal (see 
chapter 5 for further discussion). In reality, this condition is relaxed due to the finite nature of the sample, as well as deviations from monochromaticity of the incident electrons [94]. Diffraction can occur when

$$
\vec{k}_{I}-\vec{k}_{F}=\vec{g}_{h k l}+\vec{s}
$$

where $\vec{s}$ is a vector, called the deviation parameter, that measures the deviation from exact diffraction conditions.

To find the zone axis, one takes the cross products of the reciprocal vectors present (the reflections present) in the pattern. As the $g_{b k \digamma}$ vectors are perpendicular to the real-space (blel) planes, the cross products should be contained within these planes. The resulting cross product is a vector in reciprocal space; for non-cubic systems, a transformation is necessary to obtain the correct indices for the real-space zone axis. $\varepsilon$-cobalt has cubic symmetry; thus, the real space and reciprocal space vectors of the same indices point along crystallographically equivalent directions [94]. This can be easily shown using equations derived from the definitions of reciprocal space vectors (eqn 5.4).

From these definitions, we find that

$$
\vec{a} \cdot \vec{a}^{*}=\vec{b} \cdot \vec{b}^{*}=\vec{c} \cdot \vec{c}^{*}=1
$$

and

$$
\vec{a} \cdot \vec{b}^{*}=\vec{a} \cdot \vec{c}^{*}=\vec{b} \cdot \vec{a}^{*}=\vec{b} \cdot \vec{c}^{*}=\vec{c} \cdot \vec{a}^{*}=\vec{c} \cdot \vec{b}^{*}=0
$$

For a cubic system, the following is true for the unit cell vectors:

$$
\vec{a} \cdot \vec{b}=\vec{a} \cdot \vec{c}=\vec{b} \cdot \vec{c}=0
$$

In other words, due to the orthogonal nature of the cubic unit cell vectors, $\vec{a}$ is parallel to $\vec{a}^{*}$, etc., and, since $\vec{a}, \vec{b}$, and $\vec{c}$ all have the same length, $\vec{a}^{*}, \vec{b}^{*}$, and $\vec{c}^{*}$ have the same 
lengths as each other. Thus, reciprocal space and real-space vectors point in the same directions for cubic systems.

\subsection{Analysis of textured patterns from cobalt nanocrystal assemblies}

\subsubsection{Basic indexing}

Patterns from oriented and non-oriented nanocrystals were indexed (see figures 6.4, 6.6, and 6.7) by taking ratios of the ring or arc radii. Determination of the zone axis entailed taking the cross products of all possible $\langle h k l>$ corresponding to the observed $h k l$ reflections. For the pattern in 6.7 , the common result of these cross products was a $<652>$ zone. For the typical textured patterns shown in figures 6.5 and 6.6, no common zone axis was found. Cross-product results are listed in Appendix 2. This means there are probably multiple orientations present, although it is not clear whether there is merely a distribution around a given zone axis, or a fixed number of different orientations. The most common results for zone axes were $\langle 652\rangle,<110>$ and $<100>$. The dark-field images in figure 6.10 (and perhaps, those in 6.12 or 6.13 ) suggested the possibility of having three different crystal orientations within a single island. Further consideration of the 221 reflections in the SAED also indicated the possible presence of multiple domains, because, while the 221 arcs are $60^{\circ}$ apart, there are no three planes within the $\{221\}$ family that are $60^{\circ}$ apart.

In order to learn more about the crystal orientations present in the islands, the sample was tilted with a double tilt holder (figure 6.18) and SAED patterns at different tilt orientations were collected (figure 6.19). 


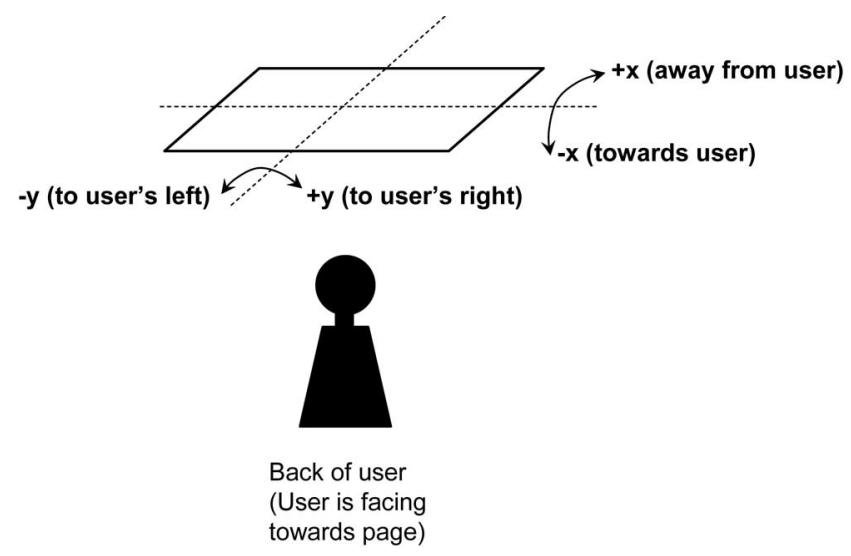

Figure 6.18. Geometry of tilting in the sample holder. 

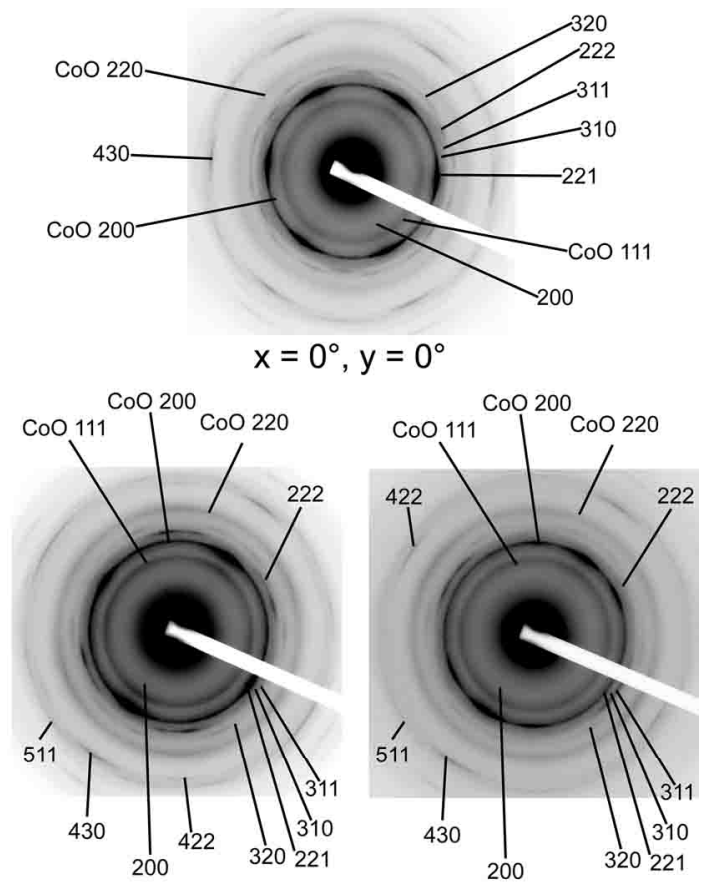

$$
x=0^{\circ}, y=-17^{\circ}
$$$$
x=0^{\circ}, y=+17^{\circ}
$$
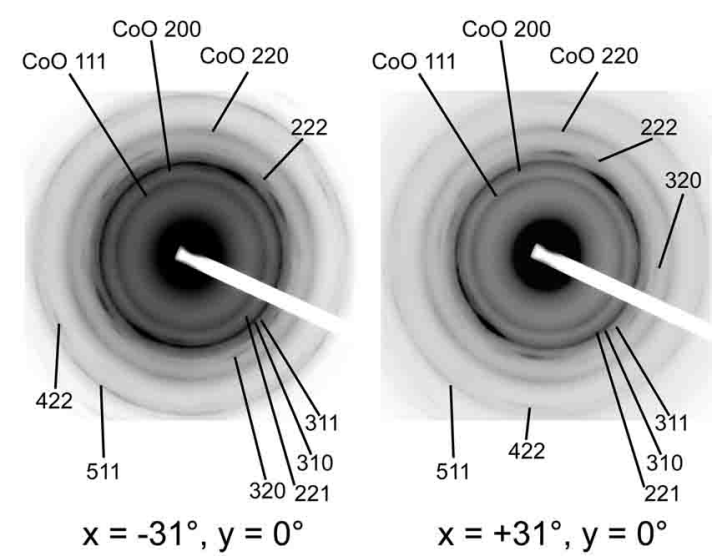

Figure 6.19. SAED from center of island at various orientations.. SAED patterns obtained at NCEM with the JEOL 3010 TEM by D. Aruguete. 


\subsubsection{Hypotheses regarding crystallographic organization within the nanocrystal} islands

As previously mentioned, dark field imaging of the assemblies suggested the presence of three populations of nanocrystals, each with a specific orientation. An early attempt was made to interpret the patterns in figures 6.5 and 6.6 using a simple model of three separate single-crystal domains, without taking any orientational distributions into account. The results from this analysis, detailed in Appendix 2, were inconclusive. Crystallographic models which account for an angular distribution are currently under consideration. These are described in the following sections.

\subsubsection{Hypothesis 1: Three separate partially-aligned domains}

One of the most common types of partial orientation seen in many polycrystalline materials is a configuration in which one crystal axis is fixed for all crystalline domains (i.e., in this discussion, the nanocrystals), but other axes vary freely [119]. The effect of this preferred orientation upon diffraction is most easily visualized in reciprocal space, as shown in figure 6.20. 


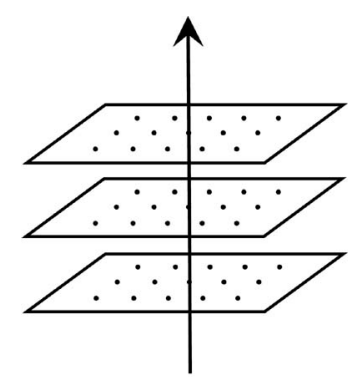

Fixed single orientation

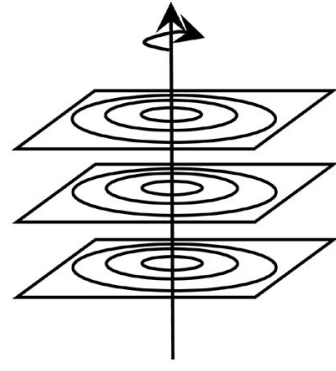

One fixed crystal axis

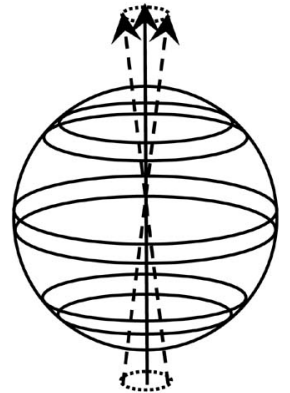

Imperfectly fixed crystal axis

Figure 6.20. Images of different types of orientation in reciprocal space.

For an ideal set of identically-aligned crystals with all axes fixed, reciprocal space is a lattice of fixed points. If only one axis of these crystals is fixed, but they are otherwise allowed rotational freedom, these points will turn into rings. Finally, if this axis is not perfectly fixed, instead of rings in reciprocal space, there will be belts. When the Ewald sphere slices through these belts, the resuting image in the diffraction pattern is that of arcs, as we see in our texture patterns.

An arrangement of three separate groups of nanocrystals, or domains, in which each domain has a single imperfectly fixed crystal axis in the sample plane might give rise to the sixfold symmetric patterns observed. This hypothesized scenario is depicted in figure 6.21. 

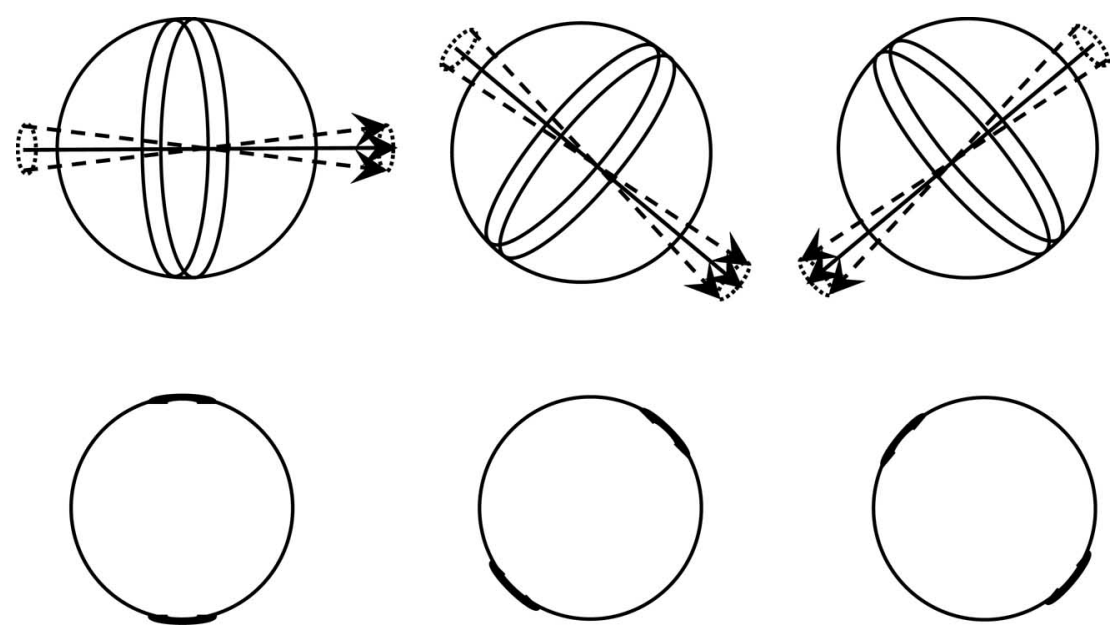

Figure 6.21. Hypothesized three-domain crystallographic arrangement of the cobalt nanocrystals in multilayered assemblies. Reciprocal-space depictions of three crystal domains (top row) and the resulting diffraction patterns (bottom row). Each domain has one imperfectly fixed axis in the sample plane. In this diagram, the Ewald sphere is the plane of this page.

\subsubsection{Hypothesis 2: Alignment along axis canted out of the sample plane}

Previous work on other polycrystalline systems with preferred crystallographic orientations suggests another possibility. The nanocrystals could all have a single partially fixed axis which is tilted out of the sample plane [120,121]. Figure 6.22 depicts how this arrangement could give rise to the observed SAED patterns. 


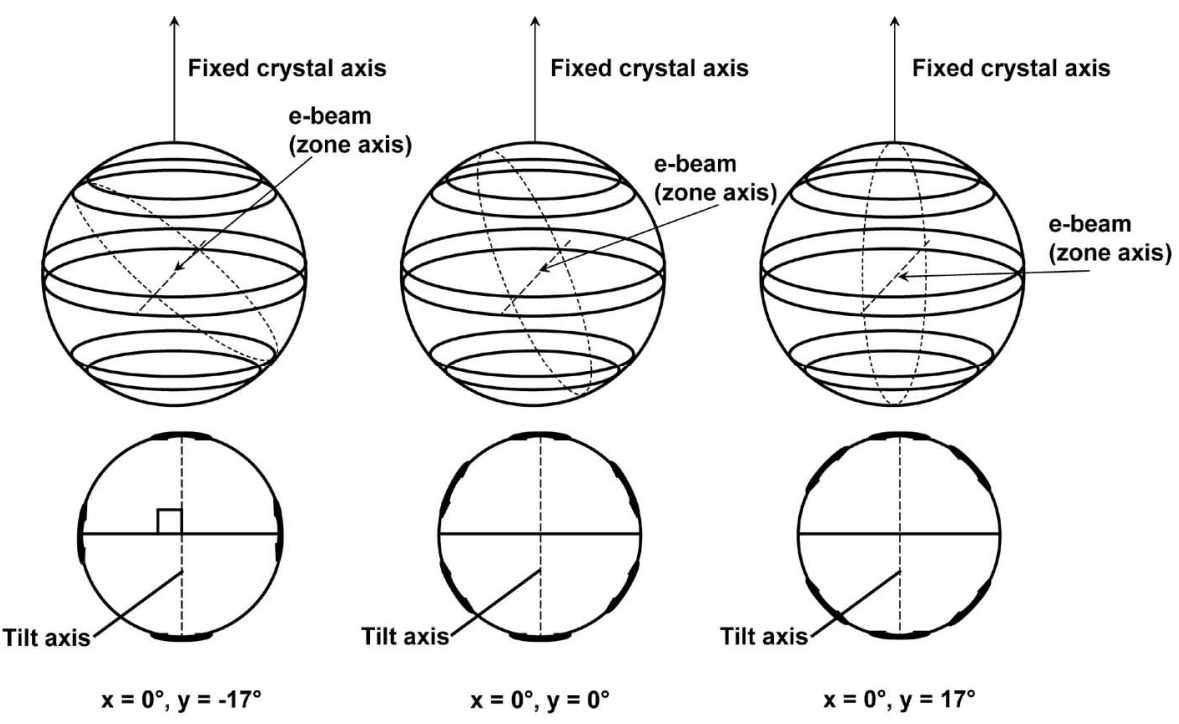

Figure 6.22. Alternative hypothesized crystallographic arrangement, in which the nanocrystals all have a single axis (imperfectly fixed) which is oriented out of the sample plane. (See figure 6.20 for further illustration of this type of preferred orientation.) Top row: Reciprocal space depictions of the proposed crystallographic arrangement at three different sample tilt settings. The dashed lines depict the tilt axis for the sample, and the dashed ellipse is the projection of the Ewald sphere. The bottom row of figures depicts the resulting diffraction patterns; they are schematics of the 221 reflections observed in the experimental SAED patterns. 
Currently, efforts are underway to computationally simulate diffraction patterns from these various possible crystallographic arrangements for comparison with our experimental data.

\subsection{Causes of crystallographic orientation in cobalt nanocrystal assemblies}

\subsubsection{Introduction}

When contemplating the drying-mediated assembly of colloidal nanocrystals, there are many factors to consider, such as particle-particle interactions, particle-solvent interactions, and particle-substrate interactions [28-30]. For colloidal magnetic nanocrystals, forces between particles are of particular interest. In addition to Van der Waals interactions, the magnetic dipoles of the nanocrystals can interact with each other. This section is a discussion of both the magnetic and van der Waals forces that may have led to the observed assemblies.

\subsubsection{Magnetism and crystallographic alignment in nanocrystals}

\subsubsection{Energetic considerations}

Magnetism is intimately coupled with the crystal structure of a material via the magnetocrystalline anisotropy energy $E_{C}$, which is the energetic stabilization gained when the dipoles of a crystal align along a given crystal axis. For magnetic ordering to translate into an alignment of the crystalline axes, the $E_{C}$ per particle must be large enough to provide an observable correlation between the magnetic dipole and crystallographic orientation. Since $\varepsilon$-Co has not been produced as a bulk phase, experimental values of the magnetocrystalline 
energy must be extracted from the magnetic relaxation kinetics of dilute nanoparticle solutions. The best value in the literature is $\sim 5 \mathrm{kT}$ for a $9.5 \mathrm{~nm} \varepsilon$-Co particle [49]. Assuming a Boltzmann distribution, this coupling gives a FWHM of $\sim 40^{\circ}$ for the angle between the easy axis and the dipole direction, somewhat larger than the FWHM of $\sim 20^{\circ}$ observed for the diffracted intensity around a peak in our SAED diffraction patterns. The discrepancy can be partially accounted for by assuming a significantly larger magnetocrystalline energy for the particles, which is reasonable given the large $( \pm 60 \%)$ estimated particle-to-particle variation for $E_{C}$ within a single sample [49]. A less rigorous estimate from previous work in the Alivisatos group gives $E_{C} \sim 7.4 \mathrm{kT}$ [122]. An undetected, slight faceting of particles might also improve alignment, reducing the FWHM of the orientation distribution function to the experimentally observed values.

Finding a possible vortex-like configuration of nanocrystals, as shown in figures 6.10 and 6.11, encouraged the consideration of the magnetic interactions between nanocrystals. Classical magnetic dipoles are the most stable when aligned end-to-end [123]; hence loopclosing configurations are an expected outcome from spontaneous dipole alignment.

To investigate the magnetic structures likely to form in lattice-based arrays of cobalt nanocrystals, a collaboration was initiated with Professor Joel Moore (physics, UCB), who conducted thermal Monte Carlo (MC) simulations on multilayer fcc crystals of dipoles. As expected, the temperature for significant ordering in an assembly was considerably reduced from the nearest-neighbor interaction energy $\left(E_{M} \sim 1500 \mathrm{~K}\right)$ both because of the angular dependence of the dipole-dipole interaction and because, in the limit of a large assembly, there are low-energy "spin-wave" fluctuations of the ordering direction that prevent spontaneous symmetry breaking in 2D systems [124]. Fig. 6.23 A shows the in-plane vortex 
structure of dipoles that appeared in the ground state $(\mathrm{T}=0)$ for a single layer of 123 dipoles. Thermal fluctuations at nonzero temperature both reduced the vorticity (quantified as the average magnitude of the sum over all dipoles of the tangential component of the dipole direction), and caused the dipoles to lie partially out-of-plane.

The primary numerical result (Fig. $6.23 \mathrm{~B}$ ) was the mean vorticity as a function of temperature for systems of 1-4 layers with 123 or 124 dipoles per layer. In cylindrical coordinates $r, \theta$, $z$, where $z$ is the out-of-plane direction, vorticity is defined as $\sum_{i}\left|n_{i} \cdot \hat{\theta}_{i}\right|$, where the sum is over all dipoles and $n$ is the local dipole direction. The temperature values assume dipole moments of $4 \times 10^{4} \mu_{\mathrm{B}}$ per particle [49] with a center-to-center spacing of 11 nm. The vorticity showed a rapid crossover but no sharp transition, as expected, and the center of this crossover occurs at around 400-500 K. Increasing layer thickness and system radius were found to sharpen the crossover and slightly lower its temperature; the largest system studied was 5 layers of 243-244 dipoles each, which shows vorticity $v>0.6$ at $\mathrm{T}=$ $390 \mathrm{~K}$. The MC simulation used a local Metropolis-type algorithm with $\sim 10^{5}$ samples per dipole. At temperatures below $400 \mathrm{~K}$, the statistical error bars were larger because the ergodic time for the assembly becomes very long.z

The simulation approximated the magnetic moment distribution within an fcc lattice as single dipole moments fixed at the center-of-mass of each nanocrystal. Thermal equilibrium was assumed to describe the configuration of these dipole moments. The actual growth process does not take place at equilibrium, but the simulational results support the conclusion that dipolar interactions between nanoparticles can result in the observed vortex ordering at room temperature. At short distances, the actual interaction between 
nanoparticles will be somewhat stronger than the pure dipole-dipole interaction we have assumed, but this difference vanishes as interparticle separation increases.

Fig. 6.23 $\mathrm{C}$ shows the squared $z_{-}^{-c o m p o n e n t ~ o f ~ t h e ~ d i p o l e ~ d i r e c t i o n ~ a s ~ a ~ f u n c t i o n ~ o f ~}$ distance from the center of a 5-layered island of 1220 dipoles, at $\mathrm{T}=389 \mathrm{~K}$. The dipoles cant out-of-plane close to the origin and near the edge, as at these points the ordering field from the other dipoles is relatively weak. The dipole loops are never perfectly circular because the dipole locations are fixed to an fcc lattice, which results in preferred directions for the dipoles. This anisotropy allows a nonvanishing sextupole moment for the dipole array, which gives the dominant average field at distances much larger than the array size. 


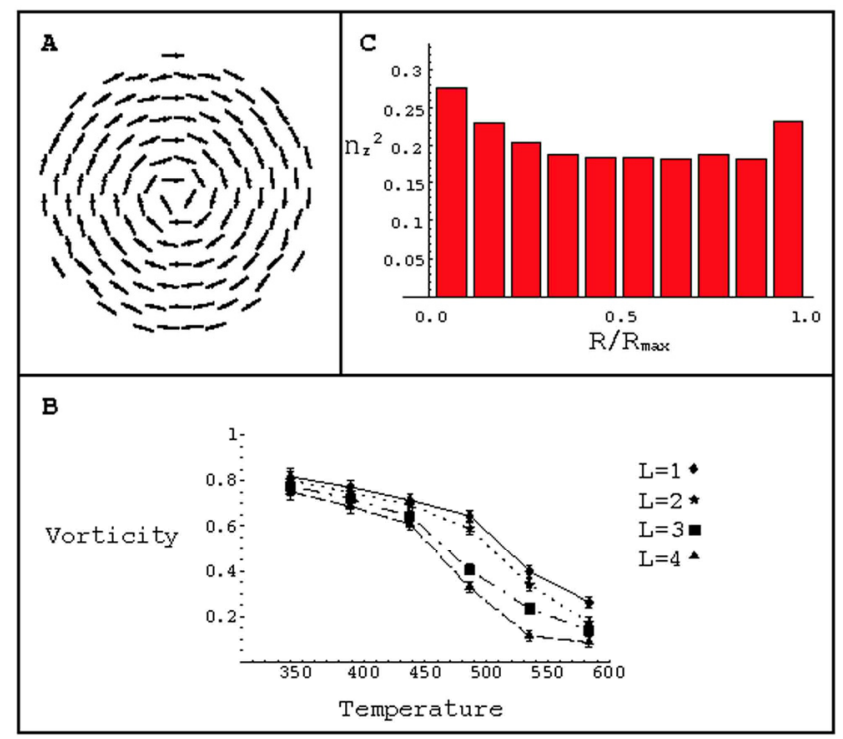

Figure 6.23. Results of energy calculations on lattices of classical dipoles. (A) Ground-state vortex arrangement of a monolayer of 123 dipoles. B) Mean vorticity versus temperature for assemblies of 1 to 4 layers with 123 or 124 dipoles per monolayer. In cylindrical coordinates $r, \theta$, $z$, where $z$ is the out-of-plane direction, vorticity is defined as $\sum_{i}\left|n_{i} \cdot \hat{\theta}_{i}\right|$, where the sum is over all dipoles and $n$ is the local dipole direction. The configuration in (A) has vorticity 0.98. (C) Mean squared $z$ component of dipole direction versus radius for a system with 5 layers and 1220 dipoles at $389 \mathrm{~K}$. 


\subsubsection{Kinetic effects of magnetism in assembly}

While the previous discussion demonstrates that a vortex configuration at room temperature may indeed be possible for an fcc lattice of nanocrystals, it does not take into account the processes of actually forming the assembly. For a nanoparticle in contact with a monolayer of particles in a vortex state, the interaction energy between its dipole and the monolayer's field is $\sim E_{M}$. Far away from the monolayer (particle-monolayer separation $r>>$ $R$, the monolayer radius), the interaction energy scales as $r^{-7}$, as the lowest nonvanishing multipole of the monolayer is the sextupole. Therefore, orientation of the particle by the monolayer field must take place very close to the monolayer, e.g. $r / d<2$, where $d$ is the particle separation in the monolayer. Using simplifying arguments, it is possible to place some kinetic constraints on the monolayer growth process for orientational order to emerge. Assuming $E_{C}>E_{M}>k T$, the correlation between the particle dipole and crystallographic coordinates becomes perfect. In that case, the magnitude of the torque orienting a particle with the monolayer, $N$, is due to the magnetic interaction energy and given by $N=-M H \sin \phi$, where $\phi$ is the angle between the position dependent magnitude of the field due to the monolayer, $H$, and the magnetic dipole, $M$, of the approaching particle. The maximum value for this torque is attained for a particle in contact with the monolayer and has magnitude $\sim E_{M}$. This torque gives rise to a rotational velocity, $\omega=N / \xi_{r}$, where $\xi_{r}$ is the rotational friction constant for the particle. The average time for the particle to rotate to the minimum energy configuration is given by $t_{R}=\langle\phi / \omega(\phi)\rangle$, with the average taken over

$\phi$. For a rigid spherical nanoparticle of diameter $d$ in a solvent of viscosity $\eta, \xi_{r}=\pi \eta d^{3}$. Using $d=11 \mathrm{~nm}$ and $\eta=1.32 \mathrm{cP}$ for 1,2-dichlorobenzene, $t_{\mathrm{R}} \sim 0.3 \mu \mathrm{s}$. For comparison, 
using $\tau=f_{0}^{-1} \exp \left(-E_{C} / k T\right)$ with $f_{0}=4 \times 10^{9} s^{-1}$ as measured for $25 \mathrm{~nm}$ fcc-Co nanoparticles [125], the lifetime of a dipole orientation in zero field, $\tau$, for a $9 \mathrm{~nm} \varepsilon$-Co particle will exceed $t_{\mathrm{R}}$ when $E_{C} \geq 7 \mathrm{kT}$. Thus by assuming a somewhat larger anistropy energy (and/or magnetic moment density) for $\varepsilon$-Co than measured previously [49], one predicts that the particle dipole is frozen to a single easy crystal axis within the timescale of particle rotation, enhancing the likelihood of successful alignment. For crystallographic alignment to emerge, each particle that attaches to a growing island must then remain free to rotate for a time exceeding $t_{\mathrm{R}}$.

A more realistic discussion of the kinetics is complicated by several factors. First, relevant energies and timescales turn out to be similar in magnitude $\left(E_{M} \sim E_{C} ; \tau \sim t_{R}\right)$. This makes it difficult to make simplifications by considering a degree of freedom to be frozen or ergodically sampled during the process. Future computational work could address in detail the mutual evolution of the crystallographic and magnetic orientation of a particle subject to thermal fluctuations [126]. Secondly, since any orientation process would have to take place when the nanoparticle is in contact with the island, short-range interactions between particles are important $[28,127]$. Finally, in the experimentally observed multilayers, the deposition process might occur in a more complicated mode or geometry than discussed above.

No crystallographic evidence of vortex formation in monolayers has been observed, even though this was predicted to be energetically favorable. The assemblies are deposited onto a carbon surface that is not rigid, and a rough surface topography could induce disorder in the first few deposited monolayers of particles. These bottom layers could then act as a 
template for the formation of more perfect close-packed layers. It is also possible that the bottom layers act as a magnetic template: the first few layers that are deposited could be orientationally disordered and the imperfect vortex field from these layers could increase the orientational order of subsequent layers.

\subsubsection{Van der Waals (or packing) forces: the role of faceting}

While faceting has been shown to influence crystallographic orientation in selfassembled nanocrystals [102], it had not originally been considered to influence the cobalt nanocrystal assembly, due to the lack of obvious facets in conventional or HRTEM.

Nevertheless, studies of the assembly of ostensibly spherical gold nanocrystals indicated that faceting may influence assembly, even when it cannot be detected with TEM. In two separate studies of surfactant-coated gold nanocrystals [103, 128], ordered assemblies were formed in which the nanocrystals had preferred crystallographic orientations (as seen in the SAED patterns); in neither study were the gold nanocrystals obviously faceted. A similar phenomenon may be occurring with the cobalt nanocrystals.

There are a few differences between these cases of facet-driven assembly and our observations [102, 103]. First, in these cases, when dark-field imaging was performed, there was no evidence of large-scale orientation of the particles into separate domains. Second, these faceted particles formed assemblies with preferred orientation even when they were in monolayers, which ours did not. It should be noted that in the case of the gold particles, multilayered assemblies displayed stronger alignment than monolayer assemblies. It is possible that we only observed ordering in multilayer assemblies due to less uniformity in our nanocrystals (hence necessitating many more "template layers" for better ordering). 
Crystallographic alignment was not always observed in the multilayered assemblies of Co nanocrystals. While this might indicate that the Co nanocrystals are not faceted, there are actually multiple documented cases of faceted particles (e.g., silver [129] and cobalt [130]) that did not display crystallographic alignment of their atomic lattices under SAED examination. Some believe that the particles may form very small aligned areas, but do not maintain this ordering over larger areas. Experimental factors beyond absolute control (e.g. varying evaporation rates, sample substrates of varying roughness altering crystal nucleation rates) may contribute to the disorder present in the assemblies formed during evaporation.

\subsection{Conclusion and outlook}

In conclusion, complex crystallographic alignments in magnetic nanocrystal assemblies were observed using a combination of TEM and SAED. In addition to measuring partial crystallographic orientation (texture) of the nanocrystals, indications of higher-level ordering, namely a crystallographic vortex phase, were also detected. Modeling of magnetic dipoles in fcc lattices revealed a vortex-like arrangement of dipoles at room temperature, which may cause the observed crystallographic alignments. Consideration of the interplay between Van der Waals and dipolar forces suggests that varying degrees of crystallographic order are possible within a limited regime of experimental parameters.

Investigation of assembly formation for different combinations of particle magnetic properties, solvent, external fields or substrate patterning could lead to a higher degree of order and to other novel structures. 


\section{BIBLIOGRAPHY}

[1] A. P. Alivisatos, Journal of Physical Chemistry 100, 13226 (1996).

[2] Y. W. Jun et al., Coord. Chem. Rev. 249, 1766 (2005).

[3] I. Gur et al., Science 310, 462 (2005).

[4] W. U. Huynh, J. J. Dittmer, and A. P. Alivisatos, Science 295, 2425 (2002).

[5] Y. M. Huh et al., J. Am. Chem. Soc. 127, 12387 (2005).

[6] M. Bruchez et al., Science 281, 2013 (1998).

[7] M. G. Manera et al., Proceedings of SPIE--The International Society for Optical Engineering 5836, 27 (2005).

[8] A. L. Efros, and M. Rosen, Annu. Rev. Mater. Sci. 30, 475 (2000).

[9] K. Jacobs, J. Wickham, and A. P. Alivisatos, J. Phys. Chem. B 106, 3759 (2002).

[10] W. T. Wallace, and R. L. Whetten, J. Phys. Chem. B 104, 10964 (2000).

[11] A. Eychmuller, J. Phys. Chem. B 104, 6514 (2000).

[12] L. R. Becerra et al., Journal of Chemical Physics 100, 3297 (1994).

[13] M. A. Marcus et al., Journal of Physical Chemistry 95, 1572 (1991).

[14] J. T. Hu et al., Science 292, 2060 (2001).

[15] L. Manna et al., Nat. Mater. 2, 382 (2003).

[16] M. A. El-Sayed, Accounts of Chemical Research 37, 326 (2004).

[17] This is not to denigrate the vast body of work on carbon nanotubes.

[18] L. Manna, E. C. Scher, and A. P. Alivisatos, J. Am. Chem. Soc. 122, 12700 (2000).

[19] L. Manna et al., Journal of Physical Chemistry B 109, 6183 (2005).

[20] K. M. Krishnan et al., J. Mater. Sci. 41, 793 (2006). 
[21] A. R. Pease, and J. F. Stoddart, in Molecular Machines and Motors (SPRINGER-

VERLAG BERLIN, Berlin, 2001), pp. 189.

[22] B. D. Terris, and T. Thomson, J. Phys. D-Appl. Phys. 38, R199 (2005).

[23] C. J. Hawker, and T. P. Russell, MRS Bull. 30, 952 (2005).

[24] V. Balzani, A. Credi, and M. Venturi, Chem.-Eur. J. 8, 5524 (2002).

[25] C. B. Murray, C. R. Kagan, and M. G. Bawendi, Annu. Rev. Mater. Sci. 30, 545 (2000).

[26] E. V. Shevchenko et al., J. Am. Chem. Soc. 124, 11480 (2002).

[27] E. V. Shevchenko et al., J. Am. Chem. Soc. 128, 3620 (2006).

[28] E. Rabani, and S. A. Egorov, Journal of Chemical Physics 115, 3437 (2001).

[29] E. Rabani et al., Nature 426, 271 (2003).

[30] J. Tang, G. L. Ge, and L. E. Brus, J. Phys. Chem. B 106, 5653 (2002).

[31] D. C. Koningsberger, and R. Prins, X-Ray Absorption: Principles, Applications, Tecbniques of EXAFS, SEXAFS, and XANES (John Wiley and Sons, 1988), Vol. 92.

[32] P. A. Tipler, Physics for Scientists and Engineers (Worth Publishers, New York, 1991), Vol. 2.

[33] D. Briggs, and M. P. Seah, Practical Surface Analysis: Auger and X-ray Photoelectron Spectroscopy (John Wiley and Sons, New York, 1996), Vol. 1, p. 674.

[34] P. A. Lee et al., Rev. Mod. Phys. 53, 769 (1981).

[35] M. N. Berberan-Santos, Journal of Chemical Education 67, 757 (1990).

[36] J. J. Rehr, and R. C. Albers, Rev. Mod. Phys. 72, 621 (2000).

[37] R. L. Liboff, Introductory Quantum Mecbanics (Addison-Wesley Publishing Co., Inc., New York, 1998). 
[38] A. L. Ankudinov et al., Physical Review B: Condensed Matter 58, 7565 (1998).

[39] Please see documentation on "k-space and background removal" program, written by Dr. Matthew Marcus, at http://xraysweb.lbl.gov/uxas/Beamline/Software/Documentation/Documentation.htm.

[40] M. A. Marcus et al., J. Synchrot. Radiat. 11, 239 (2004).

[41] L. S. Li et al., Nano Lett. 2, 557 (2002).

[42] V. F. Puntes et al., J. Am. Chem. Soc. 124, 12874 (2002).

[43] X. L. Tian, K. Chen, and G. Y. Cao, Mater. Lett. 60, 828 (2006).

[44] Y. D. Yin et al., Science 304, 711 (2004).

[45] S. M. Lee et al., J. Am. Chem. Soc. 124, 11244 (2002).

[46] C. Soennichsen, and A. P. Alivisatos, Nano Lett. 5, 301 (2005).

[47] Aihua Fu, A. P. Alivisatos, personal communication.

[48] R. A. M. Hikmet et al., Adv. Mater. 17, 1436 (2005).

[49] G. A. Held et al., Phys. Rev. B 6401 (2001).

[50] D. L. LesliePelecky, and R. D. Rieke, Chem. Mat. 8, 1770 (1996).

[51] X. G. Peng et al., Nature 404, 59 (2000).

[52] Z. A. Peng, and X. G. Peng, J. Am. Chem. Soc. 123, 1389 (2001).

[53] J. R. Mei, and V. Lemos, Solid State Communications 52, 785 (1984).

[54] G. Dalba et al., Physical Review B: Condensed Matter and Materials Physics 58, 4793 (1998).

[55] J. T. Hu et al., J. Phys. Chem. B 106, 2447 (2002).

[56] Most notably, the thesis of Dr. Liang-shi Li.

[57] L. S. Li, and A. P. Alivisatos, Adv. Mater. 15, 408 (2003). 
[58] A. Manceau, D. Chateigner, and W. P. Gates, Phys. Chem. Miner. 25, 347 (1998).

[59] A. Manceau, and M. L. Schlegel, Phys. Chem. Miner. 28, 52 (2001).

[60] A. Manceau, M. A. Marcus, and N. Tamura, in Applications of synchrotron radiation in low-temperature geochemistry and environmental science, edited by P. Fenter et al.Washington, D.C., 2002).

[61] D. P. Masson, D. J. Lockwood, and M. J. Graham, J. Appl. Phys. 82, 1632 (1997).

[62] K. S. Hamad, Ph.D. dissertation. (Department of Chemistry, University of California, Berkeley, Berkeley, CA, 2000), p. 189.

[63] M. A. Marcus, A. Manceau, and M. Kersten, Geochim. Cosmochim. Acta 68, 3125 (2004).

[64] Y. S. Touloukian et al., Thermal expansion--nonmetallic solids (Plenum, New York, 1977), Vol. 13.

[65] F. Gygi, The Qbox code, Lawrence Livermore National Laboratory, Livermore, CA.

[66] The ABINIT code is a common project of the Universite Catholique de Louvain, Corning Incorporated, and other contributors (http://www.abinit.org).

[67] A. Puzder et al., Phys. Rev. Lett. 92, 217401 (2004).

[68] C. Yeh et al., Physical Review B: Condensed Matter 46, 10086 (1992).

[69] J. Rockenberger et al., J. Phys. Chem. B 101, 2691 (1997).

[70] J.-Y. Zhang et al., Appl. Phys. Lett. 81, 2076 (2002).

[71] U. Rossler, Landolt-Bornstein--Condensed Matter: II-VI and I-VII Compounds; Semimagnetic

Compounds (Springer-Verlag GmbH, 1999), Vol. 41, p. 721.

[72] L. Manna et al., J. Am. Chem. Soc. 124, 7136 (2002).

[73] H. W. Seo et al., Journal of Chemical Physics 116, 9492 (2002). 
[74] S. W. Han et al., Appl. Phys. Lett. 86 (2005).

[75] J. Taylor, T. Kippeny, and S. J. Rosenthal, Journal of Cluster Science 12, 571 (2001).

[76] J. E. B. Katari, V. L. Colvin, and A. P. Alivisatos, Journal of Physical Chemistry 98, 4109 (1994).

[77] G. Bunker, Nucl. Instrum. Methods Phys. Res. 207, 437 (1983).

[78] E. D. Crozier, J. J. Rehr, and R. Ingalls, in X-ray absorption: Principles, applications, techniques of EXAFS, SEXAFS, and XANES, edited by D.C.Koningsberger, and R. Prins (John Wiley and Sons, New York, 1988).

[79] The interested reader is directed to the thesis of Aihua Fu (Alivisatos group, Spring 2006).

[80] M. A. Hines, and P. Guyot-Sionnest, Journal of Physical Chemistry 100, 468 (1996).

[81] G. A. Somorjai, Introduction to surface chemistry and catalysis (Wiley, New York, 1994), p. 667.

[82] J. Rockenberger et al., Journal of Chemical Physics 108, 7807 (1998).

[83] R. A. D. Pattrick, J. F. W. Mosselmans, and J. M. Charnock, European Journal of Mineralogy 10, 239 (1998).

[84] K. M. Kemner et al., Japanese Journal of Applied Physics, Part 1: Regular Papers, Short Notes \& Review Papers 32, 399 (1993).

[85] G. J. Camargo-Gamboa et al., Thin solid films 490, 165 (2005).

[86] C. Levelut et al., Materials Science and Engineering B8, 251 (1991).

[87] A. V. Baranov et al., Phys. Rev. B 68 (2003).

[88] Y. Yan et al., J. Appl. Phys. 89, 5944 (2001).

[89] T. Hakkarainen et al., J. Phys.-Condes. Matter 16, S3009 (2004). 
[90] M. Ohring, The Materials Science of Thin Films (Academic Press, San Diego, CA, 1992).

[91] Vacancy defects have been predicted to be energetically more favored to accomodate lattice mismatch for Ag/Ni heterophase boundaries. See P. Gumbsch et al., Phys. Rev. B 43, 13833 (1991).

[92] The background discussion presented in this chapter is based heavily off of the four references following this footnote.

[93] N. W. Ashcroft, and N. D. Merman, Solid State Physics (Brooks Cole, Pacific Grove, CA, 1976).

[94] D. B. Williams, and C. B. Carter, Transmission Electron Microscopy: A Textbook for Materials Science (Springer, Berlin, 2004).

[95] P. B. Hirsch, Electron microscopy of thin crystals (R.E. Krieger Publishing Co., Malabar, FL, 1977), p. 563.

[96] J. W. Edington, Practical electron microscopy for materials science (Van Nostrand Reinhold Co., New York, 1976), p. 344.

[97] Discussion in this section is primarily adapted from the following: N. W. Ashcroft, and N. D. Merman, Solid State Physics (Brooks Cole, Pacific Grove, CA, 1976).

[98] This discussion is heavily adapted from N. W. Ashcroft, and N. D. Merman, Solid State Physics (Brooks Cole, Pacific Grove, CA, 1976).

[99] Y. Cui et al., Nano Lett. 4, 1093 (2004).

[100] P. M. Mendes, and J. A. Preece, Current Opinion in Colloid and Interface Science 9, 236 (2004).

[101] J. J. Urban et al., J. Am. Chem. Soc. 128, 3248 (2006).

[102] D. F. Kang et al., Philos. Mag. Lett. 83, 569 (2003). 
[103] S. I. Stoeva et al., J. Phys. Chem. B 107, 7441 (2003).

[104] M. B. Sigman, A. E. Saunders, and B. A. Korgel, Langmuir 20, 978 (2004).

[105] T. Qiu et al., Appl. Phys. Lett. 88 (2006).

[106] A. B. Smetana, K. J. Klabunde, and C. M. Sorensen, Journal of Colloid and Interface Science 284, 521 (2005).

[107] S. H. Sun, Adv. Mater. 18, 393 (2006).

[108] V. F. Puntes, K. M. Krishnan, and P. Alivisatos, Appl. Phys. Lett. 78, 2187 (2001).

[109] M. P. Pileni et al., Faraday Discussions 125, 251 (2004).

[110] S. H. Sun, and C. B. Murray, J. Appl. Phys. 85, 4325 (1999).

[111] E. V. Shevchenko et al., Nature 439, 55 (2006).

[112] F. X. Redl et al., Nature 423, 968 (2003).

[113] C. B. Murray et al., IBM J. Res. Dev. 45, 47 (2001).

[114] J. B. Tracy et al., Phys. Rev. B 72 (2005).

[115] Y. Bao, M. Beerman, and K. M. Krishnan, Journal of Magnetism and Magnetic Materials 272-276, e1367 (2004).

[116] V. F. Puntes, K. M. Krishnan, and A. P. Alivisatos, Science 291, 2115 (2001).

[117] D. P. Dinega, and M. G. Bawendi, Angew. Chem.-Int. Edit. 38, 1788 (1999).

[118] S. L. Tripp, R. Dunin-Borkowski, and A. Wei, Angew. Chem.-Int. Edit. 42, 5591 (2003).

[119] Some scientists might refer to this type of preferred orientation as fiber texture. It is debated whether the term "fiber" can be directly applied to the system discussed in this chapter. Therefore, the term "fiber" has been omitted.

[120] L. Tang et al., Journal of Applied Crystallography 29, 419 (1996). 
[121] L. Tang, and D. E. Laughlin, Journal of Applied Crystallography 29, 411 (1996).

[122] V. F. Puntes, and K. M. Krishnan, IEEE Trans. Magn. 37, 2210 (2001).

[123] S. Chikazumi, Physics of magnetism (Wiley, New York, 1964), p. 554.

[124] N. D. Mermin, and H. Wagner, Phys. Rev. Lett. 17, 1133 (1966).

[125] W. Wernsdorfer et al., Phys. Rev. Lett. 79, 4014 (1997).

[126] J. L. Garcia-Palacios, and F. J. Lazaro, Phys. Rev. B 58, 14937 (1998).

[127] U. Landman, and W. D. Luedtke, Faraday Discussions 125, 1 (2004).

[128] L. O. Brown, and J. E. Hutchison, J. Phys. Chem. B 105, 8911 (2001).

[129] S. A. Harfenist et al., Journal of Physical Chemistry 100, 13904 (1996).

[130] Z. L. Wang, Z. R. Dai, and S. H. Sun, Adv. Mater. 12, 1944 (2000).

[131] V. F. Puntes, W. J. Parak, and A. P. Alivisatos, European Cells and Materials 3, 128 (2002). 


\section{APPENDIX 1: IMAGE PROCESSING WITH ADOBE PHOTOSHOP 7.0 (PC)}

The primary image processing performed was the adjustment of the tonal range. Here, a dark-field image of a cobalt nanocrystal assembly directly scanned from a negative is shown. The image was contrast was first inverted by using the Invert command (in the Image $\rightarrow$ Adjustments menu). The image is shown in Figure A1.

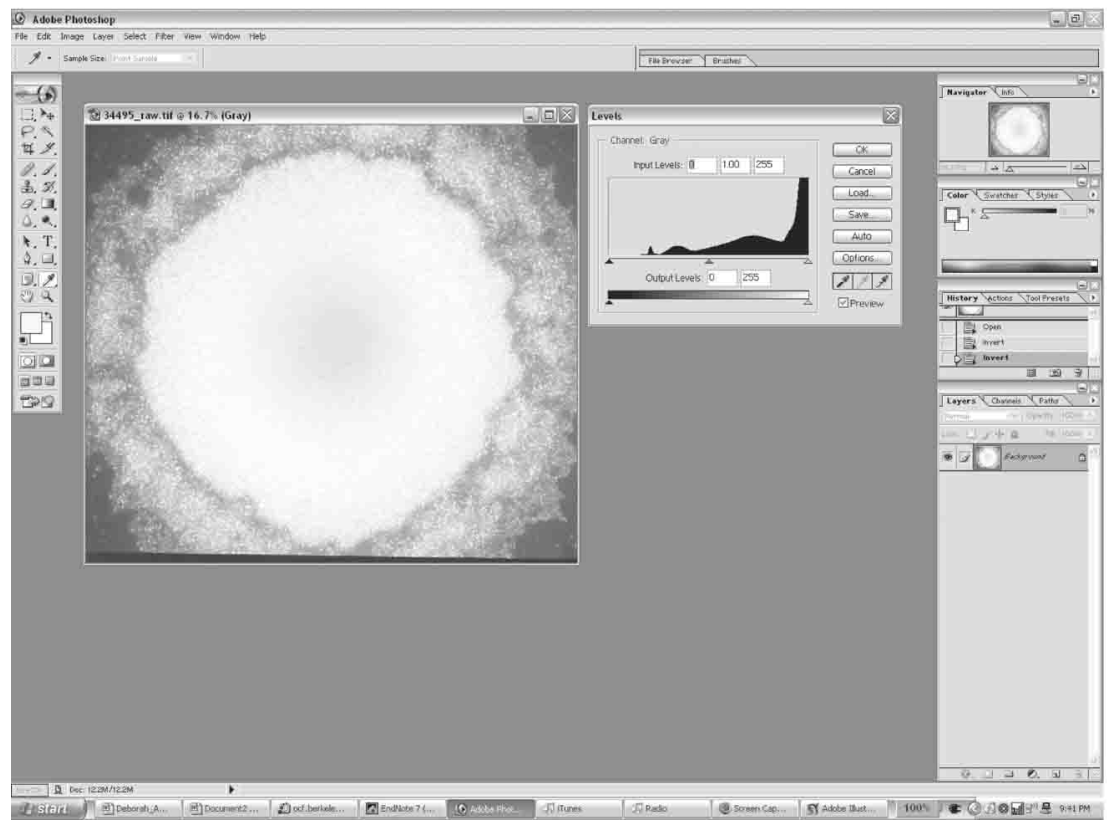

Figure A1. Screen capture of Adobe Photoshop with an overexposed dark field image of a cobalt nanocrystal island. The Levels dialogue box is shown next to the image.

The tonal range (gray levels) of the image can be inspected by using the Levels command (in the Image $\rightarrow$ Adjustments menu). A close-up of this dialogue box is shown in Figure A2. A histogram of the number of pixels for each gray level, ranging from black to 
white, is shown. In this case, the shades towards the white end of the spectrum are overrepresented, due to the overexposure of the original image.

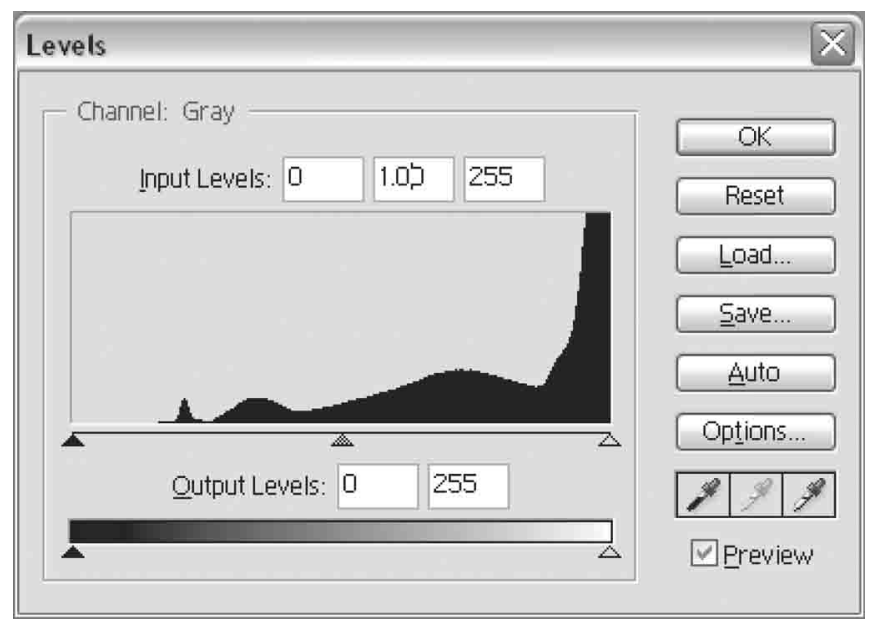

Figure A2. Screen capture image of the Levels dialogue box.

Moving the black slider on the histogram to the first group of pixels will map these pixels to black, increasing the tonal range of the image. The corresponding pixels in the other channels are adjusted proportionately to avoid altering the color balance. The middle slider is used to change the intensity values of the middle range of gray tones. A moderately-adjusted image is shown in figure A3, and the corresponding Levels dialogue box shown in Figure A4. 


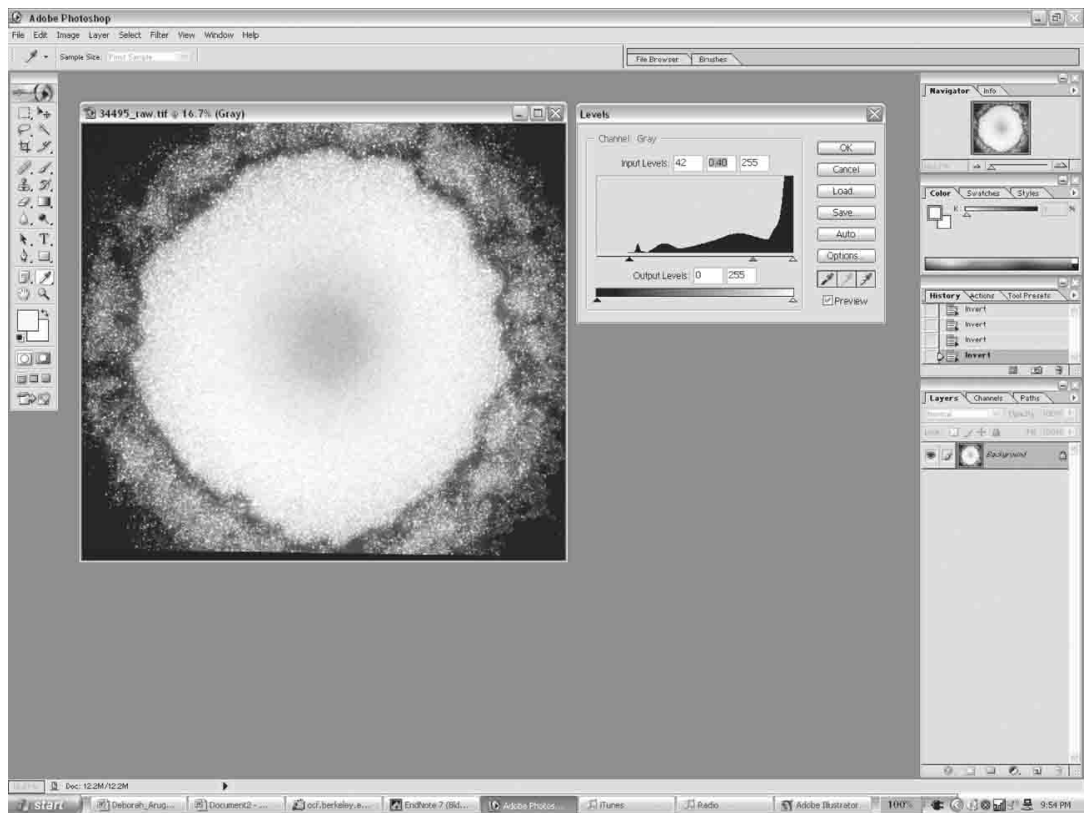

Figure A3. Dark field image with moderate adjustments of the tonal level range.

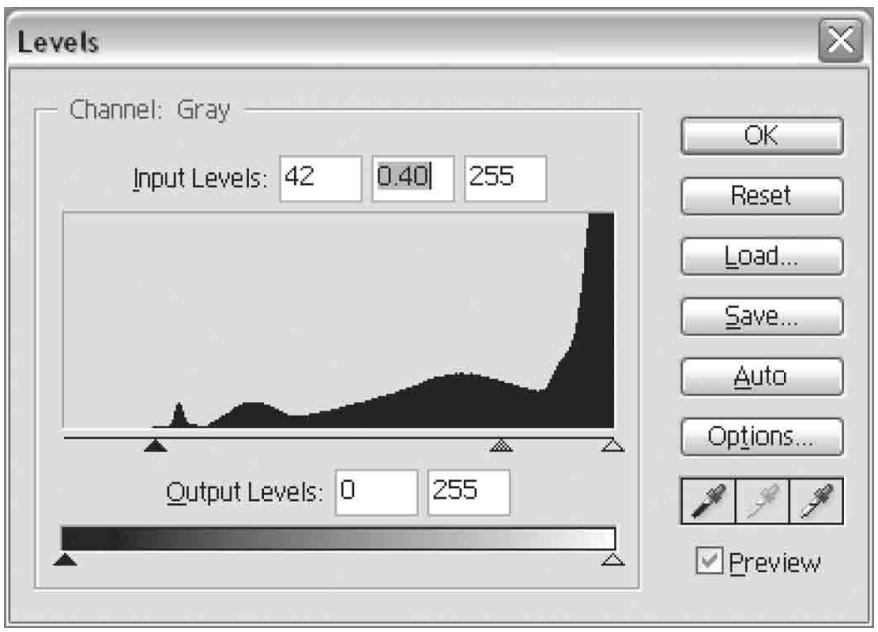

Figure A4. Close-up of Levels dialogue box with histogram. 
As this image was overexposed, a more drastic change in the tonal range is necessary to extract any useful information regarding the spatial distribution of nanocrystal orientations. This change is shown in Figure A5, with the accompanying Levels dialogue box in Figure A6. Most of the gray levels were removed from the image.

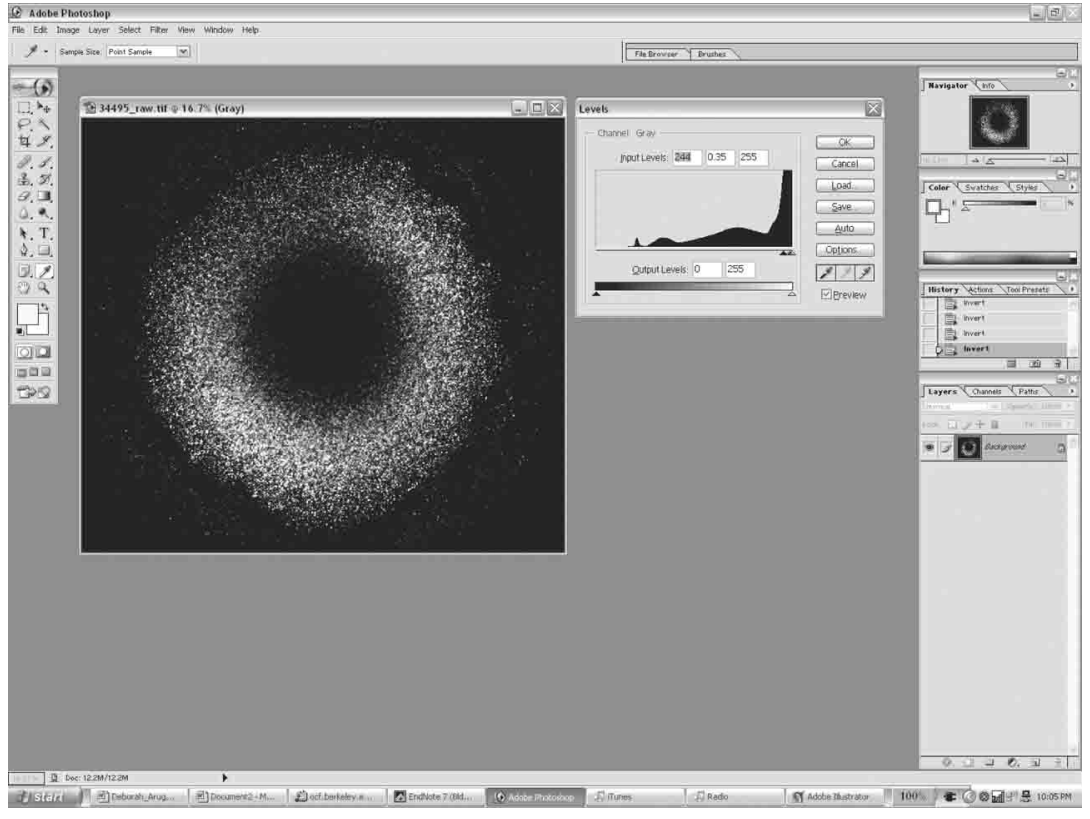

Figure A5. Final tonal range adjustment of the dark field image. 


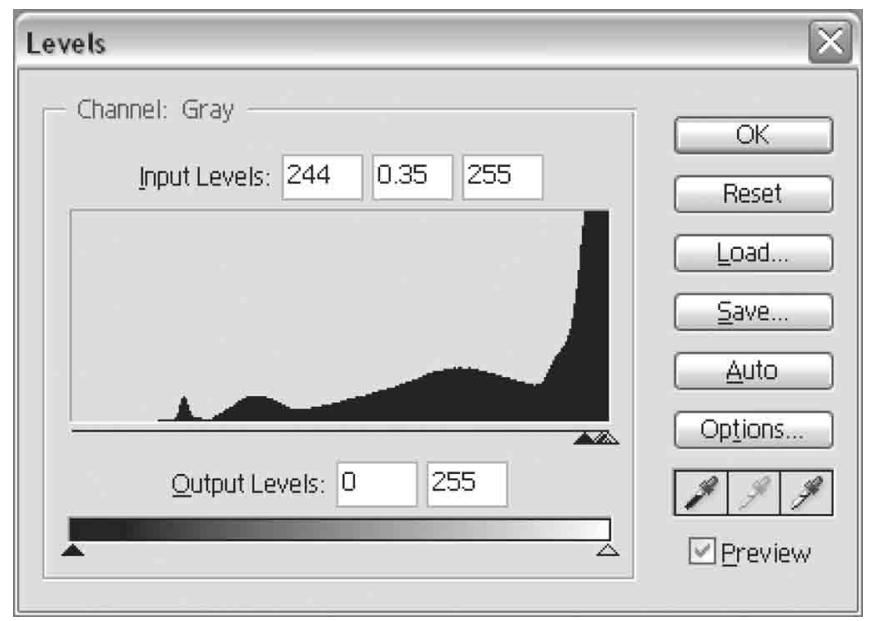

Figure A6. The corresponding Levels dialogue box for Fig. A5. Note how most of the gray levels have been discarded. 


\section{APPENDIX 2: INDEXING OF DIFFRACTION PATTERNS AND CROSS PRODUCTS OF RECIPROCAL SPACE VECTORS}

\subsection{Diffraction data for reference compounds}

Table A1: $d$-spacings and $d_{\mathrm{hkl}} / d_{221}$ ratios for $\varepsilon$-cobalt

\begin{tabular}{|c|c|c|c|c|}
\hline$h$ & $k$ & $I$ & $d(\AA)$ & $d_{h k} / d_{221}$ \\
\hline 1 & 1 & 0 & 4.31 & 2.12 \\
\hline 1 & 1 & 1 & 3.52 & 1.73 \\
\hline 2 & 1 & 0 & 2.73 & 1.34 \\
\hline 2 & 1 & 1 & 2.49 & 1.22 \\
\hline 2 & 2 & 0 & 2.16 & 1.06 \\
\hline 2 & 2 & 1 & 2.03 & 1.00 \\
\hline 3 & 1 & 0 & 1.93 & 0.95 \\
\hline 3 & 1 & 1 & 1.84 & 0.90 \\
\hline 2 & 2 & 2 & 1.76 & 0.87 \\
\hline 3 & 2 & 0 & 1.69 & 0.83 \\
\hline 3 & 2 & 1 & 1.63 & 0.80 \\
\hline 4 & 0 & 0 & 1.52 & 0.75 \\
\hline 4 & 1 & 0 & 1.48 & 0.73 \\
\hline 3 & 3 & 0 & 1.44 & 0.71 \\
\hline 3 & 3 & 1 & 1.40 & 0.69 \\
\hline 4 & 2 & 0 & 1.36 & 0.67 \\
\hline 4 & 2 & 1 & 1.33 & 0.65 \\
\hline 3 & 3 & 2 & 1.30 & 0.64 \\
\hline 4 & 2 & 2 & 1.24 & 0.61 \\
\hline 4 & 3 & 0 & 1.22 & 0.60 \\
\hline 4 & 3 & 1 & 1.20 & 0.59 \\
\hline 5 & 1 & 0 & 1.20 & 0.59 \\
\hline 5 & 1 & 1 & 1.17 & 0.58 \\
\hline 5 & 2 & 0 & 1.13 & 0.56 \\
\hline 5 & 2 & 1 & 1.11 & 0.55 \\
\hline 4 & 4 & 0 & 1.08 & 0.53 \\
\hline 5 & 2 & 2 & 1.06 & 0.52 \\
\hline 5 & 3 & 0 & 1.05 & 0.51 \\
\hline 5 & 3 & 1 & 1.03 & 0.51 \\
\hline 4 & 4 & 2 & 1.02 & 0.50 \\
\hline 6 & 1 & 0 & 1.00 & 0.49 \\
\hline 6 & 1 & 1 & 0.99 & 0.49 \\
\hline
\end{tabular}


Table A2: $d$-spacings and $d_{\mathrm{hkl}} / d_{221}$ ratios for fcc-cobalt

\begin{tabular}{|c|c|c|c|c|}
\hline $\boldsymbol{h}$ & $\boldsymbol{k}$ & $\boldsymbol{l}$ & $\boldsymbol{d}(\boldsymbol{\AA})$ & $\boldsymbol{d}_{\boldsymbol{h k} \mathbf{l}} \boldsymbol{I}_{\mathbf{2 2 1}}$ \\
\hline 1 & 1 & 1 & 2.05 & 1.01 \\
\hline 2 & 0 & 0 & 1.77 & 0.87 \\
\hline 2 & 2 & 0 & 1.25 & 0.62 \\
\hline 3 & 1 & 1 & 1.07 & 0.53 \\
\hline 2 & 2 & 2 & 1.02 & 0.50 \\
\hline
\end{tabular}

Table A3: $d$-spacings and $d_{\mathrm{hkl}} / d_{221}$ ratios for hcp-cobalt

\begin{tabular}{|c|c|c|c|c|}
\hline $\boldsymbol{h}$ & $\boldsymbol{k}$ & $\boldsymbol{l}$ & $\boldsymbol{d}(\boldsymbol{\AA})$ & $\boldsymbol{d}_{\boldsymbol{h k} \boldsymbol{l}} \boldsymbol{d}_{\mathbf{2 2 1}}$ \\
\hline 1 & 0 & 0 & 2.17 & 1.07 \\
\hline 0 & 0 & 2 & 2.03 & 1.00 \\
\hline 1 & 0 & 1 & 1.91 & 0.94 \\
\hline 1 & 0 & 2 & 1.48 & 0.73 \\
\hline 1 & 1 & 0 & 1.25 & 0.62 \\
\hline 1 & 0 & 3 & 1.15 & 0.57 \\
\hline 2 & 0 & 0 & 1.09 & 0.53 \\
\hline 1 & 1 & 2 & 1.07 & 0.52 \\
\hline 2 & 0 & 1 & 1.05 & 0.52 \\
\hline 0 & 0 & 4 & 1.02 & 0.50 \\
\hline
\end{tabular}

Table A4: $d$-spacings and $d_{\mathrm{hkl}} / d_{221}$ ratios for $\mathrm{CoO}$

\begin{tabular}{|c|c|c|c|c|}
\hline $\boldsymbol{h}$ & $\boldsymbol{k}$ & $\boldsymbol{I}$ & $\boldsymbol{d}(\AA)$ & $\boldsymbol{d}_{\boldsymbol{h k}} / \boldsymbol{d}_{\mathbf{2 2 1}}$ \\
\hline 1 & 1 & 1 & 2.46 & 1.21 \\
\hline 2 & 0 & 0 & 2.13 & 1.05 \\
\hline 2 & 2 & 0 & 1.51 & 0.74 \\
\hline 3 & 1 & 1 & 1.28 & 0.63 \\
\hline 2 & 2 & 2 & 1.23 & 0.60 \\
\hline 4 & 0 & 0 & 1.06 & 0.52 \\
\hline 3 & 3 & 1 & 0.98 & 0.48 \\
\hline
\end{tabular}


Table A5: $d$-spacings and $d_{\text {hkl }} / d_{221}$ ratios for $\mathrm{Co}_{3} \mathrm{O}_{4}$

\begin{tabular}{|c|c|c|c|c|}
\hline $\boldsymbol{h}$ & $\boldsymbol{k}$ & $\boldsymbol{l}$ & $\boldsymbol{d}(\boldsymbol{\AA})$ & $\boldsymbol{d}_{\boldsymbol{h} \boldsymbol{l}} / \boldsymbol{d}_{\mathbf{2 2 1}}$ \\
\hline 1 & 1 & 1 & 4.67 & 2.30 \\
\hline 2 & 2 & 0 & 2.86 & 1.41 \\
\hline 3 & 1 & 1 & 2.44 & 1.20 \\
\hline 2 & 2 & 2 & 2.33 & 1.15 \\
\hline 4 & 0 & 0 & 2.02 & 0.99 \\
\hline 3 & 3 & 1 & 1.85 & 0.91 \\
\hline 4 & 2 & 2 & 1.65 & 0.81 \\
\hline 3 & 3 & 3 & 1.56 & 0.77 \\
\hline 4 & 4 & 0 & 1.43 & 0.70 \\
\hline 5 & 3 & 1 & 1.37 & 0.67 \\
\hline 6 & 2 & 0 & 1.28 & 0.63 \\
\hline 5 & 3 & 3 & 1.23 & 0.61 \\
\hline 6 & 2 & 2 & 1.22 & 0.60 \\
\hline 4 & 4 & 4 & 1.17 & 0.57 \\
\hline 7 & 1 & 1 & 1.13 & 0.56 \\
\hline 6 & 4 & 2 & 1.08 & 0.53 \\
\hline 7 & 3 & 1 & 1.05 & 0.52 \\
\hline 8 & 0 & 0 & 1.01 & 0.50 \\
\hline 7 & 3 & 3 & 0.99 & 0.49 \\
\hline
\end{tabular}

\subsection{Indexing data (ratios of ring radii)}

Note that "E" stands for a reflection originating from $\varepsilon$-Co. "O" or "CoO" refers to a reflection originating from cobalt (II) oxide. While other cobalt oxides/hydroxides were considered, previous XRD data has shown that $\varepsilon$-cobalt and fcc nanocrystals form $\mathrm{CoO}$ when oxidized [44, 114, 131]. 
Table A6: Indexing of diffraction pattern from figure 6.4 (unaligned, unoxidized monolayer of nanocrystals)

\begin{tabular}{|c|c|c|}
\hline $\mathbf{r}$ (pixels) & $\mathbf{r}_{\mathbf{2 2 1}} / \mathbf{r}_{\mathbf{h k l}}$ & index \\
\hline 362.00 & 1.33 & 210 \\
\hline 398.50 & 1.21 & 211 \\
\hline 483.00 & 1.00 & 221 \\
\hline 509.00 & 0.95 & 310 \\
\hline 534.00 & 0.90 & 311 \\
\hline 602.50 & 0.80 & 321 \\
\hline 685.50 & 0.70 & 330 \\
\hline 723.50 & 0.67 & 420 \\
\hline 823.00 & 0.59 & 510 \\
\hline 869.75 & 0.56 & 520 \\
\hline 964.00 & 0.50 & 442 \\
\hline
\end{tabular}

Table A7: Indexing of diffraction pattern from figure 6.7 (multilayer of nanocrystals), $\mathrm{x}=0^{\circ}, \mathrm{y}=0^{\circ}$ stage position

\begin{tabular}{|c|c|}
\hline$r_{221} / r_{\text {hkl }}$ & index \\
\hline 1.20 & CoO $111(\mathrm{E} \mathrm{211})^{*}$ \\
\hline 1.06 & $\mathrm{CoO} 200,($ E 220)* \\
\hline 1.00 & E 221 \\
\hline 0.93 & E 310 \\
\hline 0.74 & $\mathrm{CoO} 220$ \\
\hline 0.61 & $\mathrm{CoO} 222,(\mathrm{E} 422)^{\star}$ \\
\hline 0.56 & E 520 \\
\hline 0.52 & $\mathrm{CoO} 400,(\mathrm{E} \mathrm{522})^{\star}$ \\
\hline 0.50 & E 442 \\
\hline
\end{tabular}


Table A8: Indexing of diffraction pattern from figures 6.6 and $6.19, x=0^{\circ}, y=0$ stage position

\begin{tabular}{|c|c|}
\hline$r_{221} / r_{\mathrm{hkl}}$ & index \\
\hline 1.51 & E 200 \\
\hline 1.22 & CoO 111, (E 211)* \\
\hline 1.00 & E 221 \\
\hline 0.95 & E 310 \\
\hline 0.91 & E 311 \\
\hline 0.87 & E 222 \\
\hline 0.83 & E 320 \\
\hline 0.74 & $\mathrm{CoO} 220^{*}$ \\
\hline 0.64 & E 332** \\
\hline 0.60 & $E$ 430** \\
\hline 0.58 & E 511 \\
\hline 0.56 & E 520 \\
\hline 0.54 & E 440† \\
\hline 0.50 & E 442 \\
\hline
\end{tabular}

Table A9: Indexing of diffraction pattern from figure $6.19, \mathrm{x}=0^{\circ}, \mathrm{y}=17^{\circ}$ stage position

\begin{tabular}{|c|c|}
\hline $\mathbf{r}_{\mathbf{2 2 1}} / \mathbf{r}_{\mathbf{h k l}}$ & index \\
\hline 1.49 & $\mathrm{E} \mathrm{200}$ \\
\hline 1.22 & $\mathrm{CoO} 111,(\mathrm{E} 211)^{*}$ \\
\hline 1.05 & $\mathrm{CoO} 200,(\mathrm{E} 220)^{*}$ \\
\hline 1.00 & $\mathrm{E} \mathrm{221}$ \\
\hline 0.94 & $\mathrm{E} 310$ \\
\hline 0.91 & $\mathrm{E} 311$ \\
\hline 0.87 & $\mathrm{E} 222$ \\
\hline 0.84 & $\mathrm{E} \mathrm{320}$ \\
\hline 0.74 & $\mathrm{CoO} 220^{*}$ \\
\hline 0.65 & $\mathrm{E} \mathrm{332} 2^{\text {** }}$ \\
\hline 0.62 & $\mathrm{E} 422^{* *}$ \\
\hline 0.60 & $\mathrm{E} 430^{* *}$ \\
\hline 0.58 & $\mathrm{E} 511$ \\
\hline 0.56 & $\mathrm{E} 520$ \\
\hline 0.53 & $\mathrm{E} 440 \dagger$ \\
\hline 0.50 & $\mathrm{E} 442$ \\
\hline
\end{tabular}


Table A10: Indexing of diffraction pattern from figure $6.19, x=0^{\circ}, y=-17^{\circ}$ stage position

\begin{tabular}{|c|c|}
\hline$r_{221} / r_{\text {hkl }}$ & Index \\
\hline 1.49 & E200 \\
\hline 1.23 & CoO 111, (E 211)* \\
\hline 1.05 & CoO 200, (E 220)* \\
\hline 1.00 & E 221 \\
\hline 0.95 & E 310 \\
\hline 0.91 & E 311 \\
\hline 0.87 & E 222 \\
\hline 0.83 & E 320 \\
\hline 0.74 & $\mathrm{CoO} 220^{*}$ \\
\hline 0.65 & E 332** \\
\hline 0.61 & $E 422^{* *}$ \\
\hline 0.60 & E 430** \\
\hline 0.58 & E 511 \\
\hline 0.56 & E 520 \\
\hline 0.54 & E 440† \\
\hline 0.50 & E442 \\
\hline
\end{tabular}

Table A11: Indexing of diffraction pattern from figure 6.19, $x=31^{\circ}, y=0^{\circ}$ stage position

\begin{tabular}{|c|c|}
\hline$r_{221} / r_{\mathrm{hkl}}$ & index \\
\hline 1.22 & $\mathrm{CoO} 111,(\mathrm{E} \mathrm{211})^{*}$ \\
\hline 1.06 & CoO 200, (E 220)‡ \\
\hline 1.00 & E 221 \\
\hline 0.95 & E 310 \\
\hline 0.91 & E 311 \\
\hline 0.87 & E 222 \\
\hline 0.84 & E 320 \\
\hline 0.74 & $\mathrm{CoO} 220^{*}$ \\
\hline 0.64 & E332** \\
\hline 0.61 & $\mathrm{E} 422^{* *}$ \\
\hline 0.60 & $\mathrm{E} 430^{* *}$ \\
\hline 0.58 & E511 \\
\hline 0.56 & E520 \\
\hline 0.52 & $\mathrm{E} 530^{* *}$ \\
\hline 0.50 & E442 \\
\hline
\end{tabular}


Table A12: Indexing of diffraction pattern from figure $6.19, \mathrm{x}=-31^{\circ}, \mathrm{y}=0^{\circ}$ stage position

\begin{tabular}{|c|c|}
\hline $\mathbf{r}_{\mathbf{2 2 1}} / \mathbf{r}_{\mathbf{h k l}}$ & index \\
\hline 1.23 & CoO 111, (E 211 $)^{*}$ \\
\hline 1.05 & CoO 200, (E 220) \\
\hline 1.00 & $\mathrm{E} \mathrm{221}$ \\
\hline 0.95 & $\mathrm{E} \mathrm{310}$ \\
\hline 0.91 & $\mathrm{E} 311$ \\
\hline 0.87 & $\mathrm{E} 222$ \\
\hline 0.83 & $\mathrm{E} \mathrm{320}$ \\
\hline 0.74 & $\mathrm{CoO} 220^{*}$ \\
\hline 0.64 & $\mathrm{E} 332^{* *}$ \\
\hline 0.61 & $\mathrm{E} 422^{* *}$ \\
\hline 0.59 & $\mathrm{E} 430^{* *}$ \\
\hline 0.58 & $\mathrm{E} 511$ \\
\hline 0.56 & $\mathrm{E} 520$ \\
\hline 0.53 & $\mathrm{E} 440 \dagger$ \\
\hline 0.52 & $\mathrm{E} 530^{* *}$ \\
\hline 0.50 & $\mathrm{E} 442$ \\
\hline
\end{tabular}


* While these reflections may index as both $\mathrm{CoO}$ and $\varepsilon$-cobalt reflections, it is believed that in all likelihood they are $\mathrm{CoO}$, because these particular $\varepsilon$-cobalt reflection intensities are predicted to be extremely weak relative to the $\mathrm{CoO}$ reflections.

** Some $\mathrm{CoO}$ reflections are close in position to these $\varepsilon$-cobalt reflections. What should be noted is that these reflections are strongly textured (as are the $\varepsilon$-cobalt reflections that do not coincide with $\mathrm{CoO}$ reflections). Preliminary data in early stages of analysis on nonoxidized assemblies has shown that these reflections indeed originate from $\varepsilon$-cobalt. It should also be noted that no literature could be found on oxide films with preferred crystallographic orientations forming on colloidal cobalt nanocrystals.

† This reflection was textured, but very weak, which made measurements of its position more difficult. There is some uncertainty as to the distance measured for this reflection.

$\ddagger$ The $\varepsilon$-cobalt 220 reflection is generally very weak. What is notable about the SAED pattern is that this reflection ( $\varepsilon$-cobalt 220 or $\mathrm{CoO} 200$ ) is not a continuous ring, but rather two arcs, indicating a preferred orientation. As mentioned previously, it is expected that the oxide film on the nanocrystals would not have a preferred orientation. 
Table A13. Chart of lower-index cross products for all possible $<h k l>$ corresponding to the $h k l$ reflections found in $x=0^{\circ}, y=0^{\circ}$ tilt position (SAED pattern in figure 6.18)

\begin{tabular}{|c|c|c|c|c|c|c|c|c|c|c|c|}
\hline & 200 & 221 & 310 & 311 & 222 & 320 & 332 & 430 & 511 & 520 & 440 \\
\hline 200 & & $\begin{array}{l}<110> \\
<210>\end{array}$ & $\begin{array}{l}<100> \\
<310>\end{array}$ & $\begin{array}{l}<110> \\
<310>\end{array}$ & $<110>$ & $\begin{array}{l}<100> \\
<320>\end{array}$ & $\begin{array}{l}<110> \\
<320>\end{array}$ & $\begin{array}{l}<100> \\
<430>\end{array}$ & $\begin{array}{l}<110> \\
<510>\end{array}$ & $\begin{array}{l}<100> \\
<520>\end{array}$ & $\begin{array}{l}<110> \\
<100>\end{array}$ \\
\hline 221 & & & $\begin{array}{l}<652>, \\
<631>, \\
<762>, \\
<831> \\
<431>\end{array}$ & $\begin{array}{l}\langle 110> \\
<411> \\
<534> \\
<>54> \\
\text { etc. } \\
\text { etc. }\end{array}$ & $\begin{array}{l}<110> \\
\langle 431>\end{array}$ & $\begin{array}{l}<100> \\
<322> \\
<324> \\
<641> \\
<647>\end{array}$ & $\begin{array}{l}<110> \\
<342>\end{array}$ & $\begin{array}{l}<543> \\
<431> \\
<432>\end{array}$ & $\begin{array}{l}<110> \\
<411>\end{array}$ & $\begin{array}{l}<652> \\
<542>\end{array}$ & $\begin{array}{l}<110> \\
<221> \\
<322> \\
<411>\end{array}$ \\
\hline 310 & & & & $\begin{array}{l}<310> \\
<631>\end{array}$ & $\begin{array}{l}<321> \\
<431>\end{array}$ & $\begin{array}{l}<100> \\
<321> \\
<632>\end{array}$ & $\begin{array}{l}<331> \\
<631>\end{array}$ & $\begin{array}{l}<100> \\
<431>\end{array}$ & $\begin{array}{l}<321> \\
<831>\end{array}$ & $\begin{array}{l}<652>, \\
<100>\end{array}$ & $\begin{array}{l}<100> \\
<311> \\
<331>\end{array}$ \\
\hline 311 & & & & & $\begin{array}{l}<110> \\
<211>\end{array}$ & $<332>$ & $\langle 110\rangle$ & $<543>$ & $\begin{array}{l}<110> \\
<411>\end{array}$ & $<521>$ & $\begin{array}{l}<110> \\
<211> \\
<332> \\
<411>\end{array}$ \\
\hline 222 & & & & & & $\begin{array}{l}<321> \\
<532>\end{array}$ & $\begin{array}{l}<110> \\
<651>\end{array}$ & $\begin{array}{l}<431> \\
<743>\end{array}$ & $\begin{array}{l}<110> \\
<321>\end{array}$ & $<532>$ & $\begin{array}{l}<110> \\
<211>\end{array}$ \\
\hline 320 & & & & & & & $\begin{array}{l}<320> \\
<432> \\
<643>\end{array}$ & $\begin{array}{l}<100> \\
<632>\end{array}$ & $\begin{array}{l}<321> \\
<237>\end{array}$ & $\begin{array}{l}<100> \\
<532>\end{array}$ & $\begin{array}{l}<100> \\
<322> \\
<332>\end{array}$ \\
\hline 332 & & & & & & & & $\langle 643\rangle$ & $\langle 110\rangle$ & $\begin{array}{l}\text { High } \\
\text { index }\end{array}$ & $\begin{array}{l}<110> \\
<311> \\
<331> \\
<533>\end{array}$ \\
\hline 430 & & & & & & & & & $\begin{array}{l}\text { High } \\
\text { index }\end{array}$ & $\begin{array}{l}\text { High } \\
\text { index }\end{array}$ & $\begin{array}{l}<44> \\
<433>\end{array}$ \\
\hline 511 & & & & & & & & & & $<552\rangle$ & $\begin{array}{l}<110> \\
<411> \\
<552> \\
<611>\end{array}$ \\
\hline 520 & & & & & & & & & & & $\begin{array}{l}<100> \\
<552>\end{array}$ \\
\hline 440 & & & & & & & & & & & \\
\hline
\end{tabular}

Note that "etc." refers to the fact that there were higher-index cross product results. 
Table A14. Cross products from all possible $<h k>$ corresponding to the observed $\varepsilon$ Co $h k l$ reflections. (Additional reflections were $\mathrm{CoO}$ or could not be distinguished from $\mathrm{CoO}$ with total certainty).

\begin{tabular}{|c|c|c|c|c|}
\hline & 221 & 310 & 520 & 442 \\
\hline 221 & & $\begin{array}{l}<652> \\
<631> \\
<762> \\
<831> \\
<431>\end{array}$ & $\begin{array}{l}<652>\text {, } \\
<542>\text {, } \\
\text { plus higher } \\
\text { index } \\
<\text { hkl }>\end{array}$ & $\begin{array}{l}<652>\text {, } \\
<110>\text {, } \\
<210>\text {, } \\
<432>\text {, plus } \\
\text { higher- } \\
\text { index }<\text { hkl> }\end{array}$ \\
\hline 310 & & & $\begin{array}{l}<652>\text {, } \\
<100>\text {, } \\
\text { plus higher } \\
\text { index } \\
<\text { hkl }>\end{array}$ & $\begin{array}{l}\text { Same as } \\
\text { for } 221\end{array}$ \\
\hline 520 & & & & $\begin{array}{l}\text { Same as } \\
\text { for } 221\end{array}$ \\
\hline 442 & & & & \\
\hline
\end{tabular}




\section{APPENDIX 3: INITIAL ATTEMPTS TO VERIFY A THREE-DOMAIN HYPOTHESIS FOR THE NANOCRYSTAL ASSEMBLIES}

The objective of this analysis was to find an arrangement of the nanocrystals' crystallographic orientations that would explain the results of the tilting experiments. As detailed in chapter 6 , it was clearly established that no single crystallographic orientation could cause the diffraction pattern, indicating that multiple crystallographic orientations were present. The dark field images had suggested that three crystallographic orientations were dominant amongst the nanocrystals.

With the dark field in mind, it was hypothesized that three crystal orientations were present in the sample. The model tested was the simplest picture possible of such a system. The sample was approximated as consisting of three single-crystalline domains, each of which had a $<221>$-type vector fixed in the sample plane. The angular distributions of the nanocrystals were not taken into account. This model is depicted in figure A7 for the $x=0^{\circ}$, $y=0^{\circ}$ (non-tilted) orientation, where the vectors $\vec{a}, \vec{b}$, and $\vec{c}$, which all have the same magnitudes, represent the $<221>$-type vectors. The tilt axis is coincident with the vector $\vec{a}$.

After tilting to the $x=0^{\circ}, y=-17^{\circ}$ position (Fig. 6.19), it was observed that the positions of the 221 reflections shifted to be $90^{\circ}$ from each other. The question to ask was the following: could this resulting pattern be consistent with the proposed 3-domain hypothesis? In other words, was it possible to find another $<221>$-type vector $\vec{d}$ in any of the domains that would intersect with the Ewald sphere after a $17^{\circ}$ tilt? Answering this question merely reduces to solving for the angular relationships between all $<221>$-type vectors, easily done with dot products or a stereographic projection. 

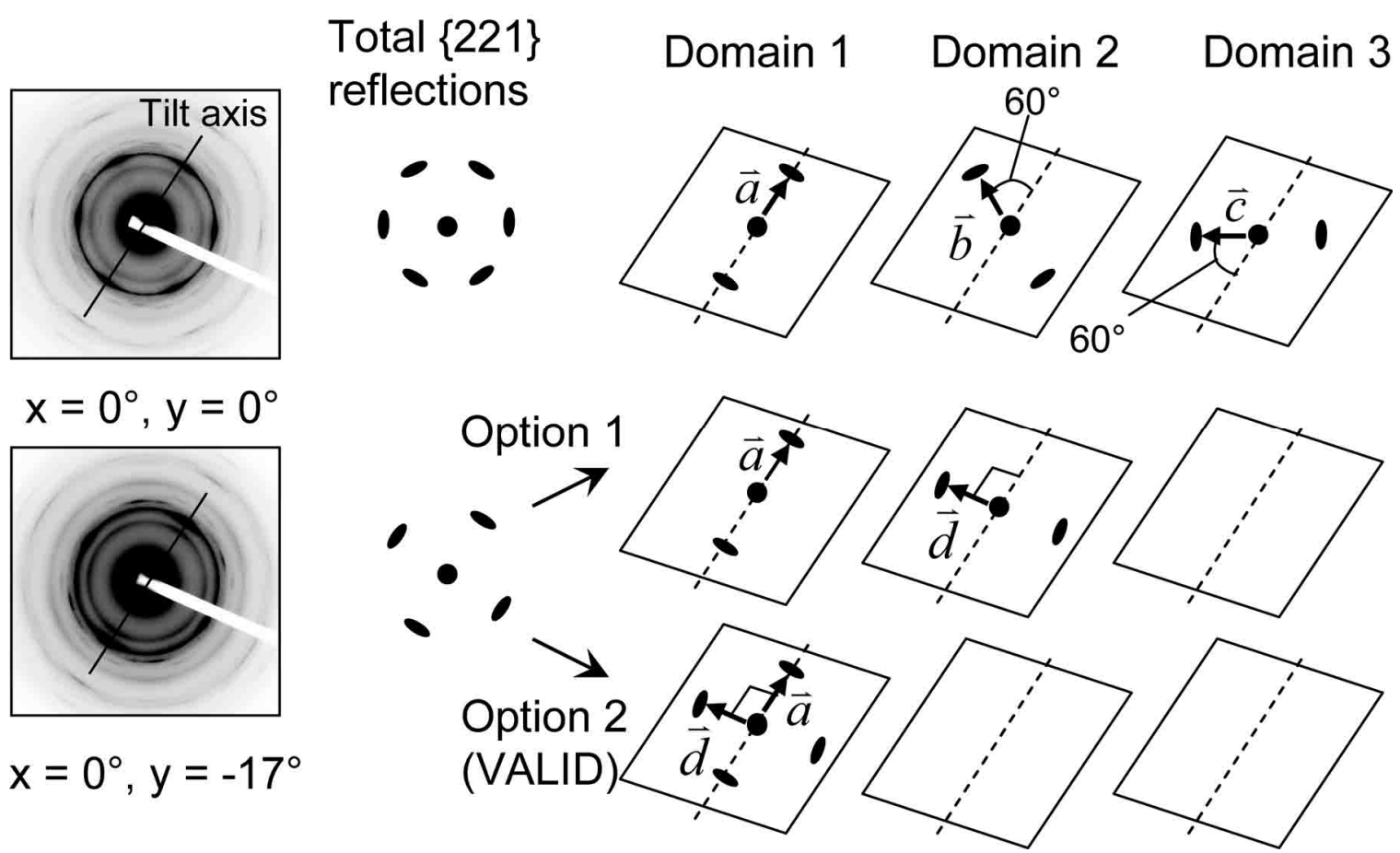

Figure A7. Possible 3 crystal domain schemes for obtaining the diffraction patterns observed at $x$ $=0^{\circ}, y=0^{\circ}$ and $x=0^{\circ}, y=-17^{\circ}$. The tilt axis is delineated on the domains with dotted lines.

Figure A7 displays the possible contributions of domains 1 and 2 to the $x=0^{\circ}, y=-$ $17^{\circ}$ diffraction pattern. (Domain 3 was also considered in this analysis but its contribution is not shown in figure A1.) In option 1, domain 2 gives rise to the vector $\vec{d}$. However, there are no two sets of $<221>$-type vectors with the proper angular relationship for this to occur (that is, $\vec{b}$ (or $\vec{c}$ ) and $\vec{d}$ cannot co-exist in a single domain). For option $2, \vec{d}$ arises upon tilting from domain 1 . This is possible because there are $<221>$-type vectors that are orthogonal, e.g. [122] and [-22-1] (the dot product is zero).

The next part of this analysis to consider was to look at the $x=0^{\circ}, y=+17^{\circ} \mathrm{SAED}$ pattern, shown in figure A9. The goal again was to find other $<221>$-type vectors that 
would have the angular relationships necessary to produce the $x=0^{\circ}, y=+17^{\circ}$ pattern.

Option 2 (figure A8) was found to be a valid possibility. A similar analysis was performed using the $x= \pm 31^{\circ}, y=0^{\circ}$ patterns, and a 3 -domain arrangement of the nanocrystals was found to be possible.

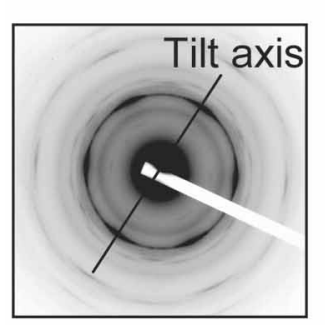

\section{Total $\{221\}$} reflections Domain 1 Domain 2 Domain 3

$x=0^{\circ}, y=0^{\circ}$
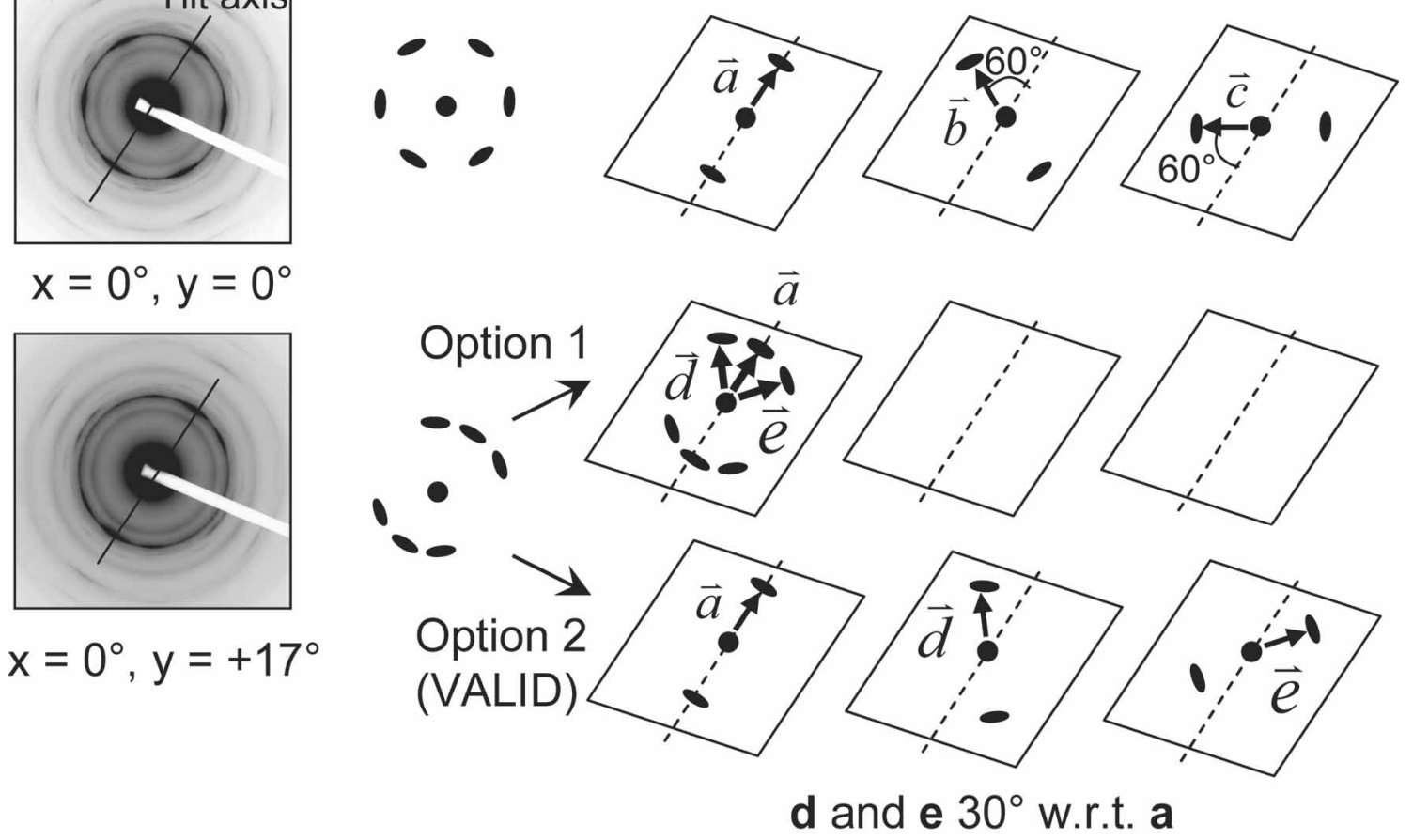

Figure A8. Possible 3 crystal domain schemes for obtaining the diffraction patterns observed at $x$ $=0^{\circ}, y=0^{\circ}$ and $x=0^{\circ}, y=+17^{\circ}$. The tilt axis is delineated on the domains with dotted lines.

Having partially shown that a system with three separate crystallographic orientations might be possible, an attempt was made to derive zone axes at the $x=0^{\circ}, y=0^{\circ}$ position for these proposed crystal domains. The approach was to use basic linear algebra and geometry to derive zone axes for the proposed crystal domains. Unfortunately, resulting derived zone 
axes did not easily factor out to being vectors of the integer form [UVW]. The analysis was likely complicated by the presence of a distribution of orientations. 